\title{
An Optical Transmission Spectrum for the Ultra-hot Jupiter WASP-121b Measured with the Hubble Space Telescope
}

Evans, Thomas M.; Sing, David K.; Goyal, Jayesh M.; Nikolov, Nikolay; Marley, Mark S.; Zahnle, Kevin; Henry, Gregory W.; Barstow, Joanna K.; Alam, Munazza K.; Sanz-Forcada, Jorge

Total number of authors:

27

Published in:

Astrophysical Journal

Link to article, DOI:

10.3847/1538-3881/aaebff

Publication date:

2018

Document Version

Publisher's PDF, also known as Version of record

Link back to DTU Orbit

Citation (APA):

Evans, T. M., Sing, D. K., Goyal, J. M., Nikolov, N., Marley, M. S., Zahnle, K., Henry, G. W., Barstow, J. K., Alam, M. K., Sanz-Forcada, J., Kataria, T., Lewis, N. K., Lavvas, P., Ballester, G. E., Ben-Jaffel, L., Blumenthal, S. D., Bourrier, V., Drummond, B., Muñoz, A. G., ... Williamson, M. H. (2018). An Optical Transmission Spectrum for the Ultra-hot Jupiter WASP-121b Measured with the Hubble Space Telescope. Astrophysical Journal, 156(6), [283]. https://doi.org/10.3847/1538-3881/aaebff

\section{General rights}

Copyright and moral rights for the publications made accessible in the public portal are retained by the authors and/or other copyright owners and it is a condition of accessing publications that users recognise and abide by the legal requirements associated with these rights.

- Users may download and print one copy of any publication from the public portal for the purpose of private study or research.

- You may not further distribute the material or use it for any profit-making activity or commercial gain

- You may freely distribute the URL identifying the publication in the public portal 


\title{
An Optical Transmission Spectrum for the Ultra-hot Jupiter WASP-121b Measured with the Hubble Space Telescope
}

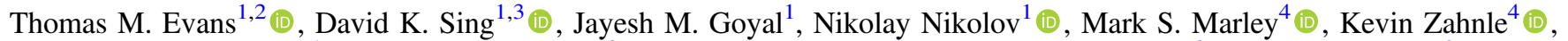 \\ Gregory W. Henry ${ }^{5}$ (i), Joanna K. Barstow ${ }^{6}$ (i) , Munazza K. Alam ${ }^{7}$ (i), Jorge Sanz-Forcada ${ }^{8}$ (i), Tiffany Kataria ${ }^{9}$ (i), \\ Nikole K. Lewis ${ }^{10}$ (D) , Panayotis Lavvas ${ }^{11}$, Gilda E. Ballester ${ }^{12}$, Lotfi Ben-Jaffel ${ }^{13}$ (D), Sarah D. Blumenthal ${ }^{1}$ (D), \\ Vincent Bourrier ${ }^{14}$ (10), Benjamin Drummond ${ }^{1}(\mathbb{B})$, Antonio García Muñoz ${ }^{15}$ (1) , Mercedes López-Morales ${ }^{7}$ (1) , Pascal Tremblin ${ }^{16}$ (D), \\ David Ehrenreich $^{14}$ (1) , Hannah R. Wakeford ${ }^{17}$ (10), Lars A. Buchhave ${ }^{18}$ (D), Alain Lecavelier des Etangs ${ }^{13}$, Éric Hébrard ${ }^{1}$ (i), and \\ Michael H. Williamson ${ }^{5}$ \\ ${ }^{1}$ Physics and Astronomy, Stocker Road, University of Exeter, Exeter, EX4 3RF, UK; tmevans@mit.edu \\ ${ }^{2}$ Kavli Institute for Astrophysics and Space Research, Massachusetts Institute of Technology, 77 Massachusetts Avenue, 37-241, Cambridge, MA 02139, USA \\ ${ }^{3}$ Department of Earth and Planetary Sciences, Johns Hopkins University, Baltimore, MD, USA \\ ${ }^{4}$ NASA Ames Research Center, Moffett Field, California, USA \\ ${ }^{5}$ Center of Excellence in Information Systems, Tennessee State University, Nashville, TN 37209, USA \\ ${ }^{6}$ Department of Physics and Astronomy, University College London, Gower Street, London WC1E 6BT, UK \\ ${ }^{7}$ Harvard-Smithsonian Center for Astrophysics, 60 Garden Street, Cambridge, MA 02138, USA \\ ${ }^{8}$ Centro de Astrobiología (CSIC-INTA), ESAC Campus, Camino Bajo del Castillo, E-28692 Villanueva de la Canada, Madrid, Spain \\ ${ }^{9}$ NASA Jet Propulsion Laboratory, 4800 Oak Grove Drive, Pasadena, CA 91109, USA \\ ${ }^{10}$ Department of Astronomy and Carl Sagan Institute, Cornell University, 122 Sciences Drive, 14853, Ithaca, NY, USA \\ ${ }^{11}$ Groupe de Spectrométrie Moléculaire et Atmosphérique, Université de Reims, Champagne-Ardenne, CNRS UMR F-7331, France \\ ${ }^{12}$ Lunar and Planetary Laboratory, University of Arizona, Tucson, AZ 85721, USA \\ ${ }^{13}$ Sorbonne Universités, UPMC Université Paris 6 and CNRS, UMR 7095, Institut d'Astrophysique de Paris, 98 bis boulevard Arago, F-75014 Paris, France \\ ${ }^{14}$ Observatoire astronomique de l'Université de Genève, 51 chemin des Maillettes, 1290 Sauverny, Switzerland \\ ${ }^{15}$ Zentrum für Astronomie und Astrophysik, Technische Universität Berlin, Hardenbergstrasse 36, D-10623 Berlin, Germany \\ ${ }^{16}$ Maison de la Simulation, CEA, CNRS, Université Paris-Sud, UVSQ, Université Paris-Saclay, F-91191 Gif-sur-Yvette, France \\ ${ }^{17}$ Space Telescope Science Institute, 3700 San Martin Drive, Baltimore, MD 21218, USA \\ ${ }^{18}$ DTU Space, National Space Institute, Technical University of Denmark, Elektrovej 328, DK-2800 Kgs. Lyngby, Denmark \\ Received 2018 July 24; revised 2018 October 22; accepted 2018 October 23; published 2018 November 28
}

\begin{abstract}
We present an atmospheric transmission spectrum for the ultra-hot Jupiter WASP-121b, measured using the Space Telescope Imaging Spectrograph on board the Hubble Space Telescope. Across the $0.47-1 \mu \mathrm{m}$ wavelength range, the data imply an atmospheric opacity comparable to-and in some spectroscopic channels exceeding - that previously measured at near-infrared wavelengths $(1.15-1.65 \mu \mathrm{m})$. Wavelength-dependent variations in the opacity rule out a gray cloud deck at a confidence level of $3.7 \sigma$ and may instead be explained by VO spectral bands. We find a cloud-free model assuming chemical equilibrium for a temperature of $1500 \mathrm{~K}$ and a metal enrichment of 10-30 $\times$ solar matches these data well. Using a free-chemistry retrieval analysis, we estimate a VO abundance of $-6.6_{-0.3}^{+0.2}$ dex. We find no evidence for $\mathrm{TiO}$ and place a $3 \sigma$ upper limit of -7.9 dex on its abundance, suggesting TiO may have condensed from the gas phase at the day-night limb. The opacity rises steeply at the shortest wavelengths, increasing by approximately five pressure scale heights from 0.47 to $0.3 \mu \mathrm{m}$ in wavelength. If this feature is caused by Rayleigh scattering due to uniformly distributed aerosols, it would imply an unphysically high temperature of $6810 \pm 1530 \mathrm{~K}$. One alternative explanation for the short-wavelength rise is absorption due to SH (mercapto radical), which has been predicted as an important product of non-equilibrium chemistry in hot Jupiter atmospheres. Irrespective of the identity of the NUV absorber, it likely captures a significant amount of incident stellar radiation at low pressures, thus playing a significant role in the overall energy budget, thermal structure, and circulation of the atmosphere.
\end{abstract}

Key words: methods: observational - planets and satellites: atmospheres - planets and satellites: gaseous planets

\section{Introduction}

Spectroscopic observations made during the primary transit of an exoplanet allow the atmospheric transmission spectrum of the day-night boundary region to be probed (Seager \& Sasselov 2000), while the same type of observation made during secondary eclipse provides the emission spectrum of the dayside hemisphere (Seager \& Sasselov 1998). Much of the transmission and emission spectroscopy work published to date has employed the Hubble Space Telescope (HST), primarily with the Space Telescope Imaging Spectrograph (STIS), covering the $0.1-1 \mu \mathrm{m}$ UV-optical wavelength range, and the Wide Field Camera 3 (WFC3), covering the $0.8-1.65 \mu \mathrm{m}$ near-IR wavelength range.
A non-exhaustive list of $H S T$ transmission spectroscopy highlights at optical through IR wavelengths include the detection of $\mathrm{Na}$ on HD 209458b (Charbonneau et al. 2002), multiple detections of $\mathrm{H}_{2} \mathrm{O}$ (e.g., Deming et al. 2013; Huitson et al. 2013; Fraine et al. 2014; Kreidberg et al. 2015; Evans et al. 2016; Wakeford et al. 2017, 2018; Tsiaras et al. 2018), widespread evidence for aerosols (e.g., Pont et al. 2008; Kreidberg et al. 2014; Nikolov et al. 2014, 2015; Sing et al. 2015, 2016), and a detection of $\mathrm{He}$ in the extended atmosphere of WASP-107b (Spake et al. 2018). At UV wavelengths, transit observations made with STIS have probed the hydrogen exospheres of hot Jupiters (e.g., Vidal-Madjar et al. 2003) and warm Neptunes (e.g., Ehrenreich et al. 2015), while heavier 
elements such as oxygen have been detected using the HST Cosmic Origins Spectrograph (e.g., Fossati et al. 2010; BenJaffel \& Ballester 2013). For emission, a similar list includes detections of $\mathrm{H}_{2} \mathrm{O}$ absorption (Stevenson et al. 2014; Beatty et al. 2017), evidence for $\mathrm{H}_{2} \mathrm{O}$ emission (Evans et al. 2017), evidence for $\mathrm{TiO}$ emission (Haynes et al. 2015), constraints on optical reflection spectra (Evans et al. 2013; Bell et al. 2017), and multiple featureless thermal spectra (e.g., Mansfield et al. 2018; Nikolov et al. 2018).

This paper reports a transmission spectrum measured for the ultra-hot $\left(T_{\text {eq }} \gtrsim 2500 \mathrm{~K}\right)$ Jupiter WASP-121b across the $0.3-1 \mu \mathrm{m}$ wavelength range using STIS. Discovered by Delrez et al. (2016), WASP-121b orbits a moderately bright $(V=10.5)$ F6V host star, which has an estimated radius of $1.458 \pm 0.030 R_{\odot}$ (Delrez et al. 2016) and measured parallax of $3.676 \pm 0.021$ mas (Gaia Collaboration et al. 2018), corresponding to a system distance of $272.0 \pm 1.6$ parsec. WASP-121b itself has a mass of $1.18 \pm 0.06 M_{\mathrm{J}}$, an inflated radius of $\sim 1.7 R_{\mathrm{J}}$, and a dayside equilibrium temperature above $2400 \mathrm{~K}$. Together, these properties make WASP-121b an excellent target for atmospheric characterization (Delrez et al. 2016; Evans et al. 2016, 2017).

We previously published the near-IR $1.15-1.65 \mu \mathrm{m}$ transmission spectrum for WASP-121b measured using WFC3 in Evans et al. (2016). Those data revealed absorption due to the $\mathrm{H}_{2} \mathrm{O}$ band centered at $1.4 \mu \mathrm{m}$, along with a second bump across the $1.15-1.3 \mu \mathrm{m}$ wavelength range, which we suggested could be a signature of $\mathrm{FeH}$ or VO. Analyzing the same data set, Tsiaras et al. (2018) reproduced the $1.15-1.3 \mu \mathrm{m}$ feature and presented a best-fit model including absorption by $\mathrm{TiO}$ and VO, although they did not discuss FeH. In Evans et al. (2016), we also compared the WFC3 transmission spectrum with transits measured at optical wavelengths by Delrez et al. (2016) using ground-based photometry. This comparison implied significantly deeper transits at optical wavelengths relative to the near-IR, which we speculated could be evidence for a strong opacity source such as $\mathrm{TiO}$ and/or VO. Subsequent modeling of these data confirmed such an interpretation to be plausible (e.g., Kempton et al. 2017; Parmentier et al. 2018).

In Evans et al. (2017), we presented a secondary eclipse observation for WASP-121b, also made with WFC3 at near-IR wavelengths. The measured spectrum indicates a mean photosphere temperature of approximately $2700 \mathrm{~K}$ and shows the $1.4 \mu \mathrm{m} \mathrm{H}_{2} \mathrm{O}$ band in emission, rather than absorption, implying the dayside hemisphere has a vertical thermal inversion. As for the transmission spectrum, the emission data exhibit a second bump across the $1.15-1.3 \mu \mathrm{m}$ wavelength range, which can be fit with $\mathrm{VO}$ in emission. To do so, however, requires assuming a VO abundance over $1000 \times$ higher than expected for solar elemental composition in chemical equilibrium, casting doubt on this interpretation. Models assuming chemical equilibrium and abundances closer to solar do not reproduce the 1.15-1.3 $\mu \mathrm{m}$ bump (e.g., Parmentier et al. 2018). For now, we do not have a satisfactory explanation for this feature, but the fact that it has been observed in both the transmission spectrum and emission spectrum is intriguing.

Our understanding of the atmosphere of WASP-121b remains a work in progress. For instance, the thermal inversion measured for the dayside hemisphere implies significant heating at low pressures ( $\lesssim 100$ mbar), though it is unclear what causes this. One possibility is absorption of incident stellar radiation at optical wavelengths by $\mathrm{TiO}$ and VO (e.g., Hubeny et al. 2003; Fortney et al. 2008). However, neither of these species have yet been definitively detected in the atmosphere of WASP-121b, despite the hints described above. Furthermore, it has been pointed out that $\mathrm{TiO}$ and $\mathrm{VO}$ could be removed from the upper atmospheres of even very hot planets by cold-trapping (e.g., Showman et al. 2009; Spiegel et al. 2009; Beatty et al. 2017). Additionally, the dayside temperatures of ultra-hot Jupiters such as WASP-121b are likely high enough for significant thermal dissociation of $\mathrm{TiO}$ and $\mathrm{VO}$, along with other molecules such as $\mathrm{H}_{2} \mathrm{O}$, to occur (Arcangeli et al. 2018; Kreidberg et al. 2018; Lothringer et al. 2018; Parmentier et al. 2018). Nonetheless, evidence for $\mathrm{TiO}$ has been detected on the dayside of WASP-33b (Haynes et al. 2015; Nugroho et al. 2017), which has a mean photosphere temperature of around $3000 \mathrm{~K}$ at near-IR wavelengths, making it even hotter than WASP-121b. An optical transmission spectrum measured for another ultra-hot Jupiter, WASP-19b, also exhibits a prominent $\mathrm{TiO}$ band (Sedaghati et al. 2017), although this may have been the signature of unocculted star spots (Espinoza et al. 2018). Despite the picture remaining unclear, observations such as these imply that $\mathrm{TiO}$, and presumably $\mathrm{VO}$, can perhaps persist at low pressures in ultrahot Jupiter atmospheres. As will be described in the following sections, the STIS transmission spectrum for WASP-121b provides new evidence for VO absorption at optical wavelengths.

Absorption at UV wavelengths may also play a significant role in heating the upper atmospheres of strongly irradiated planets such as WASP-121b. For instance, Zahnle et al. (2009) examined non-equilibrium sulfur chemistry in the context of hot Jupiter atmospheres and concluded that $\mathrm{SH}$ and $\mathrm{S}_{2}$ could be important absorbers across the $0.24-0.4 \mu \mathrm{m}$ wavelength range. These species may be driven to higher-than-equilibrium abundances via reactions involving the photolytic and photochemical destruction of $\mathrm{H}_{2} \mathrm{~S}$. As will be reported below, the measured transmission spectrum for WASP-121b exhibits a strong signal at wavelengths shortward of $\sim 0.47 \mu \mathrm{m}$ and absorption by $\mathrm{SH}$ appears to provide a viable explanation.

We begin, however, by describing our observations and the steps taken to extract the spectra from the raw data frames in Section 2. We present analyses of the white light curves in Section 3 and the spectroscopic light curves in Section 4. The results are discussed in Section 5, including the implications of the measured transmission for the planetary atmosphere. Our conclusions are given in Section 6.

\section{Observations and Data Reduction}

We observed three primary transits of WASP-121b using HST/STIS as part of the Panchromatic Comparative Exoplanet Treasury (PanCET) survey (Program 14767; P.I.s Sing and López-Morales). This comprised two visits made on 2016 October 24 and 2016 November 6 with the G430L grating, and one visit made on 2016 November 12 with the G750L grating. In what follows, we shall refer to the first and second G430L visits as the G430Lv1 and G430Lv2 data sets, respectively. For all three STIS visits, the target was observed for $6.8 \mathrm{hr}$, covering five consecutive $H S T$ orbits. Observations were made using the widest available slit $(52 \times 2$ arcsec $)$ to minimize slit losses and the detector gain was set to $4 e^{-1} / \mathrm{DN}$. Overheads were reduced by only reading out a $1024 \times 128$ pixel subarray containing the target spectrum. Exposure times of $253 \mathrm{~s}$ and $161 \mathrm{~s}$ were used for the G430L and G750L observations, respectively. We also took a short $1 \mathrm{~s}$ exposure at the start of 

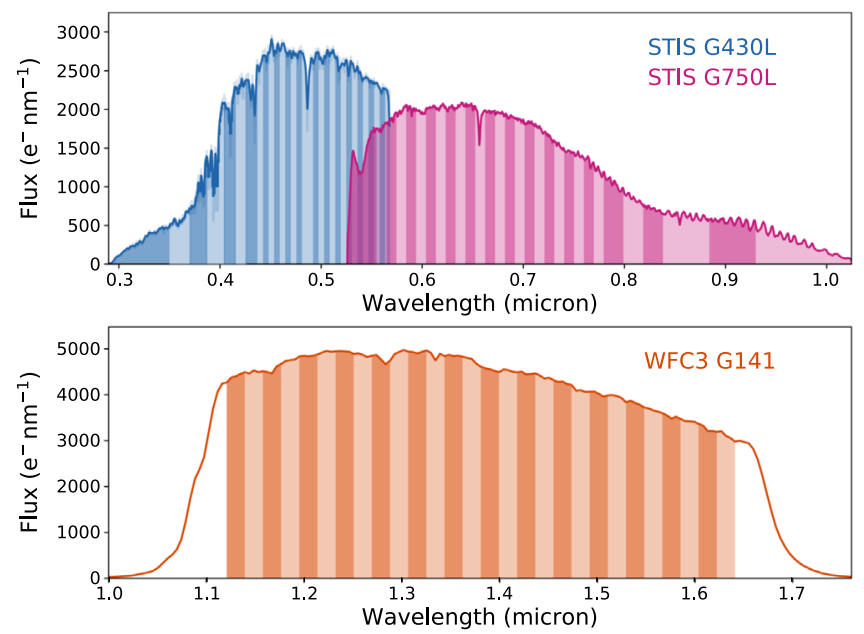

Figure 1. Example spectra for the G430L and G750L gratings (top panel) and the G141 grism (bottom panel). Dark and light vertical bands indicate the wavelength channels adopted for the spectroscopic light curves.

each HST orbit for both gratings, but discarded these exposures in the subsequent analysis. This was done because STIS observations typically suffer from a systematic in which the first exposure of each HST orbit has anomalously lower counts relative to the immediately following exposures (e.g., Evans et al. 2013; Nikolov et al. 2014, 2015; Sing et al. 2015), and we wanted to minimize the integration time lost to this effect. With this observing setup, we acquired a total of 48 science exposures for each G430L visit and 70 science exposures for the G750L visit.

The STIS data sets were reduced following the methodology described in Nikolov et al. (2014, 2015). Raw data frames were bias-, dark-, and flat-corrected using the CALSTIS pipeline (v3.4) with relevant calibration frames. Cosmic-ray events and pixels flagged as "bad" by CALSTIS were removed and interpolated over. Overall, we found $\sim 4 \%$ of pixels were affected by cosmic rays for all visits with a further $\sim 5 \%$ flagged as bad by CALSTIS. To extract spectra from the cleaned 2D frames, we used the IRAF procedure apall with aperture radii of $4.5,6.5,8.5$, and 10.5 pixels for both the G430L and G750L data sets. The dispersion axis was mapped to a wavelength solution using the $\mathrm{x} 1 \mathrm{~d}$ files produced by CALSTIS.

In addition to the STIS data, a single primary transit of WASP-121b was observed on 2016 February 6 with the G141 grism (program 14468; P.I. Evans). This data set was originally published in Evans et al. (2016), to which the reader is referred for further details.

Example G430L, G750L, and G141 spectra are shown in Figure 1.

\section{White Light Curve Analyses}

White light curves were constructed for each data set by summing the flux of each spectrum across the full dispersion axis. The resulting light curves are shown in the top row of Figure 2. As in our previous works (Evans et al. 2013, 2016, 2017), we followed the methodology outlined by Gibson et al. (2012) and treated each light curve as a Gaussian process (GP). Under this approach, the data likelihood is described by a multivariate normal distribution of the form $\mathcal{N}(\boldsymbol{d} \mid \boldsymbol{\mu}, \boldsymbol{K}+\Sigma)$, where $\boldsymbol{d}$ is an $N$-length vector containing the flux measurements, $\boldsymbol{\mu}$ is a vector containing the deterministic mean function, $\boldsymbol{K}$ is an $N \times N$ matrix describing the correlations between data points, and $\boldsymbol{\Sigma}$ is an $N \times N$ diagonal matrix containing the squared white-noise uncertainties, $\sigma_{j}^{2}$, for each data point $j=1, \ldots, N$.

For the mean function, we adopted a Mandel \& Agol (2002) transit model multiplied by a linear trend in time $(t)$ of the form $c_{0}+c_{1} t$. We assumed a circular orbit with a period $(P)$ of 1.2749255 days (Delrez et al. 2016). We allowed the normalized planet radius $\left(R_{\mathrm{p}} / R_{\star}\right)$ and transit mid-time $\left(T_{\mathrm{mid}}\right)$ to vary as free parameters with uniform priors. As described in Section 3.1, we first performed fits with the normalized semimajor axis $\left(a / R_{\star}\right)$ and impact parameter $(b)$ allowed to vary as free parameters, both with uniform priors. Then, as described in Section 3.2, we fixed $a / R_{\star}$ and $b$ to their weighted-mean values and repeated the fitting.

In all fits, we assumed a quadratic limb-darkening law and treated both coefficients $\left(u_{1}, u_{2}\right)$ as free parameters. We first estimated values for $u_{1}$ and $u_{2}$ by fitting to the limb-darkening profile of a stellar model over the appropriate bandpass. Specifically, we used a 3D stellar model from the STAGGER grid (Magic et al. 2013) with $T_{\star}=6500 \mathrm{~K}, \log _{10} g=4 \mathrm{cgs}$, and $[\mathrm{Fe} / \mathrm{H}]=0$ dex, as this was the grid point closest to the properties of the WASP-121 host star $\left(T_{\star}=6460 \pm 140 \mathrm{~K}\right.$, $\log _{10} g=4.242 \pm 0.2 \mathrm{cgs},[\mathrm{Fe} / \mathrm{H}]=+0.13 \pm 0.09 \mathrm{dex} ;$ Delrez et al. 2016). We then applied broad normal priors to $u_{1}$ and $u_{2}$ in the model fitting, with means set to these estimated values and standard deviations of 0.6 , providing plenty of flexibility for the model to be optimized.

For the GP covariance matrix $\boldsymbol{K}$, we adopted a squaredexponential kernel ${ }^{19}$ with three input variables, which are reasonably assumed could correlate with the instrumental systematics, namely, the HST orbital phase $(\phi)$, dispersion drift $(x)$, and cross-dispersion drift $(y)$. This resulted in four free parameters for each data set, namely, the covariance amplitude $(A)$ and correlation length scales $\left(L_{k}\right)$ for each input variable, $k=\{\phi, x, y\}$. For the white-noise matrix, $\Sigma$, we adopted the formal photon noise values $\sigma_{j}$ multiplied by a rescaling factor $(\beta)$, which was allowed to vary as a free parameter. The latter affords some flexibility to handle high-frequency systematics that are pseudo-white-noise in nature, which would otherwise bias the model toward impractically small $L_{k}$ values.

For the GP covariance amplitude $A$, we adopted Gamma priors of the form $p(A) \propto e^{-100 A}$, to favor smaller correlation amplitudes. This can help prevent a small number of outliers having a disproportionate influence on the inferred covariance amplitude. For the correlation length scales $L_{k}$, we followed previous studies (e.g., Evans et al. 2017; Gibson et al. 2017) and fit for the natural logarithm of the inverse correlation length scales $\ln \eta_{k}=\ln L_{k}^{-1}$, adopting uniform priors for each. In practice, this favors longer correlation length scales, with the intention of capturing the lowerfrequency systematics present in the data, as these are most degenerate with the planet signal. Higher-frequency systematics can be accounted for through the $\beta$ parameter, for which we adopted a normal prior with mean of 1 and standard deviation of 0.2 to favor values close to the formal photon noise.

We modeled the white light curves for G430L, G750L, and G141 separately. For the G430L light curves, we assumed

\footnotetext{
19 We refer the reader to previous studies such as Gibson et al. (2012), Evans et al. (2013), and Gibson (2014) for further details of the squared-exponential kernel.
} 

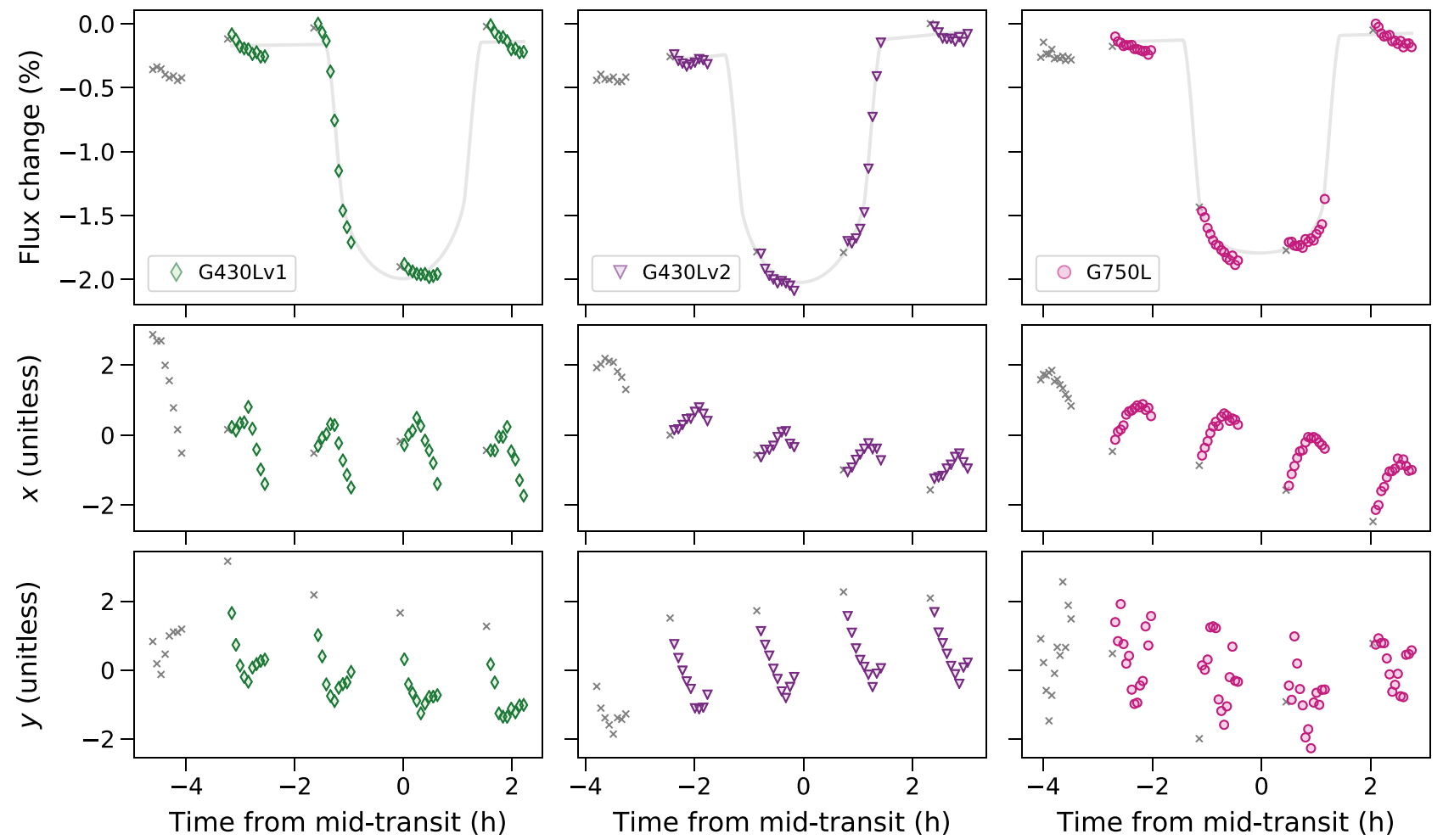

Figure 2. (Top row) Raw white light curves for the G430Lv1, G430Lv2, and G750L data sets. Gray lines show the best-fit transit signals with linear baseline trends. (Middle row) Dispersion drift variable for each data set. (Bottom row) Cross-dispersion drift variable for each data set. In all panels, colored symbols indicate data points that were included in the analysis, and gray crosses indicate those that were excluded for reasons explained in the main text. The two drift variables are unitless as they have been standardized, i.e., mean subtracted and normalized by their standard deviations.

$R_{\mathrm{p}} / R_{\star}, a / R_{\star}$, and $b$ were the same for both visits, while allowing $T_{\mathrm{mid}}, \beta, A, \ln \eta_{\phi}, \ln \eta_{x}$, and $\ln \eta_{y}$ to vary separately for each visit. The posterior distributions were marginalized using affine-invariant Markov chain Monte Carlo (MCMC), as implemented by the emcee Python package (Foreman-Mackey et al. 2013). In all fits, we randomly distributed five groups of 150 walkers throughout the parameter space and allowed them to run for 100 steps to locate the peak of the posterior distribution. We then re-initialized the five groups of 150 walkers in a tighter ball around this peak and allowed them to run for 500 steps, of which we discarded the first 250 steps as burn-in and combined the remaining 250 steps into a single chain for each walker group. At this point, a comparison of the chains from each walker group confirmed that they appeared well mixed and converged, with Gelman-Rubin statistic values within $2 \%$ of unity for each free parameter (Gelman \& Rubin 1992). Table 1 summarizes the resulting posterior distributions. For each of the STIS light curves produced using the different trial apertures (see Section 2), we obtained results consistent to within $1 \sigma$ for the planet parameters (e.g., $R_{\mathrm{p}} / R_{\star}$ ) and report only those for the 8.5 pixel aperture.

\section{1. $a / R_{\odot}$ and $b$ Allowed to Vary}

The purpose of the model fits in which $a / R_{\star}$ and $b$ were allowed to vary as free parameters was to use the HST data to refine our estimates of these system properties. Previously, the only published measurements were those provided in the original discovery paper by Delrez et al. (2016), which reported $a / R_{\star}=3.754_{-0.028}^{+0.023}$ and $b=0.160_{-0.042}^{+0.040}$. Figure 3 shows the posterior distributions obtained from our analyses for comparison, with values reported in Table 1 . We find good agreement for both $a / R_{\star}$ and $b$ across our fits to the G430L, G750L, and G141 white light curve data sets. Taking the arithmetic weighted mean of these results, we estimate $a / R_{\star}=3.86 \pm$ 0.02 and $b=0.06 \pm 0.04$, implying $i=89.1 \pm 0.5 \mathrm{deg}$. We note that our $H S T$ results differ from those of Delrez et al. by $3.5 \sigma$ for $a / R_{\star}$ and $2 \sigma$ for $b$. The reason for this disagreement is unclear and will likely be resolved by additional transit observations that are currently planned or in the process of being analyzed (T. M. Evans et al. 2018, in preparation). For the present study, we note that the primary consequence of assuming slightly different values for $a / R_{\star}$ and $b$ will be to perturb the inferred values for $R_{\mathrm{p}} / R_{\star}$. Importantly, this will be a wavelength-independent effect and thus should not affect our interpretation of the atmospheric transmission spectrum. For this reason, and given the mutual agreement between the G430L, G750L, and G141 data sets, we adopt the HST weighted-mean values for $a / R_{\star}$ and $b$ in all subsequent light curve fits.

\section{2. $a / R_{\odot}$ and $b$ Held Fixed}

Inferred values for $R_{\mathrm{p}} / R_{\star}$ can be biased by differences in the assumed values for $a / R_{\star}$ and $b$ across data sets. For this reason, we held the latter parameters fixed to the $H S T$ weighted-mean values determined in the previous section and repeated the white light curve analyses. This is physically motivated by the fact that the true values of $a / R_{\star}$ and $b$ should be constant across our data sets, and we are primarily interested in wavelength-dependent variations of $R_{\mathrm{p}} / R_{\star}$ arising due to the planetary atmosphere.

Figure 4 shows the best-fit transit models compared with the data after removing the systematics contribution inferred by the 
Table 1

Results of White Light Curve MCMC Analyses

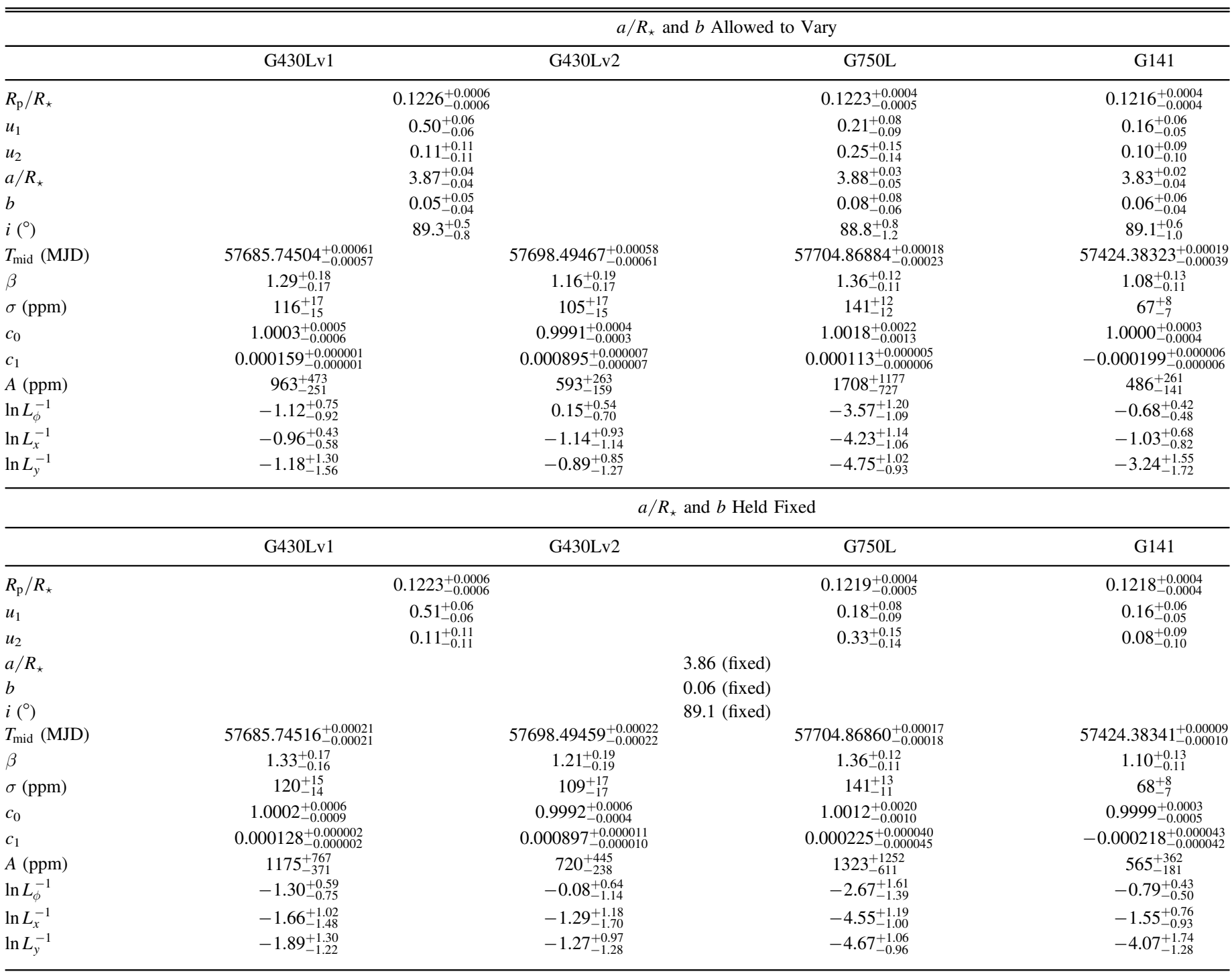

Note. Quoted values give sample medians with uncertainties corresponding to ranges encompassing $68 \%$ of samples about the median. Note that $\sigma$ values were not fit directly as part of the MCMC analysis but obtained by multiplying the $\beta$ values by the formal photon noise values for each light curve.

GP. The latter are plotted separately in Figure 5. Posterior distributions are shown in Figure 6 and summarized in Table 1. The resulting estimates for $R_{\mathrm{p}} / R_{\star}, u_{1}$, and $u_{2}$ are all within $1 \sigma$ of those obtained for the fits in which $a / R_{\star}$ and $b$ were allowed to vary. Unsurprisingly, we obtain similar estimates for $\beta$, as this parameter is sensitive to high-frequency noise in the data that is unlikely to be significantly correlated with $a / R_{\star}$ and $b$. The inferred $\beta$ values imply scatters that are $\sim 20 \%-40 \%$ and $\sim 10 \%$ above the photon noise floor for the STIS and WFC3 data sets, respectively. This is illustrated in Figure 4, which shows the model residuals. For $T_{\text {mid }}$, we find the inferred values shift by $\sim 5-20 \mathrm{~s}$, but remain within $\sim 1 \sigma$ of those obtained for the fits in which $a / R_{\star}$ and $b$ were allowed to vary.

\section{Spectroscopic Light Curve Analyses}

Spectroscopic light curves were constructed by first summing the spectra of each data set within the wavelength channels shown in Figure 1. Median channel widths were 20 pixels $(\sim 55 \AA)$ for both $\mathrm{G} 430 \mathrm{~L}$ data sets, 20 pixels $(\sim 98 \AA)$ for the G750L data set, and 4 pixels $(\sim 186 \AA)$ for the G141 data set. Care was taken to avoid the edges of prominent stellar lines and to maintain similar levels of flux within each channel. Thus, subsets of the G430L and G750L channels were broader than these nominal widths. The resulting raw light curves for the STIS data sets are shown in Figures 18-20.

We next generated common-mode (i.e., wavelength-independent) signals for each data set by dividing the raw white light curves by the corresponding best-fit transit signals obtained in Section 3 and shown in Figure 4. Each of the raw spectroscopic light curves were then divided by the resulting common-mode signals. Note that in addition to removing common-mode systematics, this latter step also has the effect of dividing each spectroscopic light curve by the intrinsic scatter of the white light curve. However, this is acceptable, as the spectroscopic light curves have a larger intrinsic scatter than the white light curves: dividing white noise by lower-amplitude white noise should on average have a zero net effect on the scatter of the resulting corrected light 


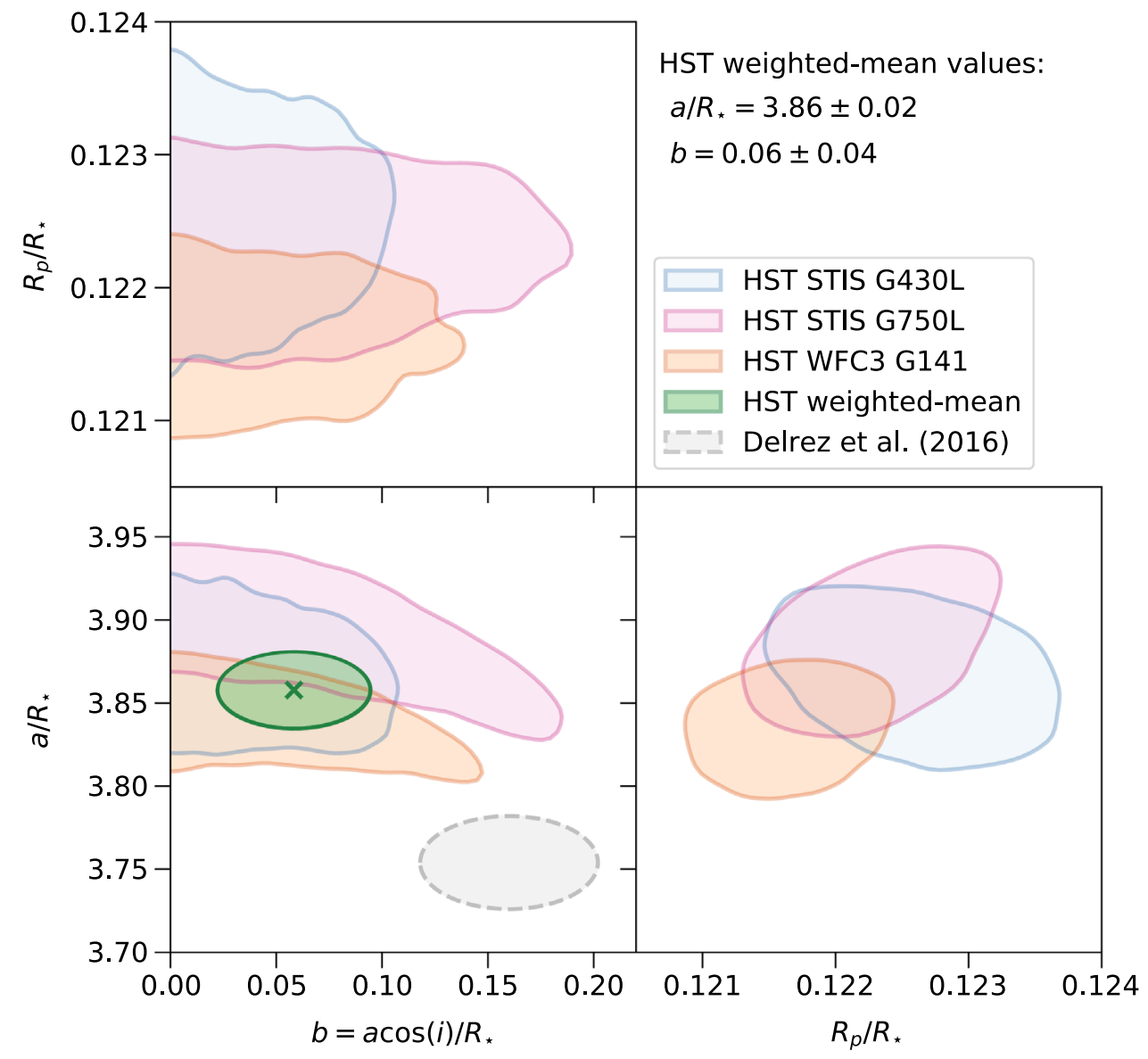

Figure 3. Posterior distributions for $R_{\mathrm{p}} / R_{\star}, a / R_{\star}$, and $b$ obtained from the white light curve analyses described in Section 3.1. The blue, pink, and orange regions indicate smoothed contours containing $68 \%$ of the MCMC samples for the G430L, G750L, and G141 analyses, respectively. The green region indicates the weighted mean of the HST posterior distributions, and the gray region indicates the $1 \sigma$ range reported by Delrez et al. (2016).

curves. Meanwhile, applying a common-mode correction of this nature-as opposed to dividing through by the best-fit systematics model from the white light curve fits-has the potential advantage of removing systematics in the spectroscopic light curves that may not be captured by our white light curve systematics model. The common-mode corrected light curves for the STIS data sets are shown in Figures 21-23.

To fit the spectroscopic light curves, we used the same approach as described in Section 3. The only exception was that we fixed $T_{\text {mid }}$ to the best-fit values listed in Table 1 . Thus, for the spectroscopic transit signals, the free parameters were the radius ratio $\left(R_{\mathrm{p}} / R_{\star}\right)$ and quadratic limb-darkening coefficients $\left(u_{1}, u_{2}\right)$. For the G430L analysis, we fit both visits jointly with shared values for $R_{\mathrm{p}} / R_{\star}, u_{1}$, and $u_{2}$, as was done for the white light curve analysis. In all fits, we again accounted for systematics by fitting for a linear trend in $t$ and a GP with $\{\phi, x, y\}$ as inputs to a squared-exponential covariance kernel. White-noise levels were allowed to vary for each individual light curve via $\beta$ rescaling parameters. Marginalization of the posterior distributions was performed in the manner described above, using affine-invariant MCMC.

The best-fit transit signals and model residuals are shown in Figure 7 for G430L and Figure 8 for G750L. Figure 9 shows the systematics and GP fits for each spectroscopic light curve. Histograms of residuals are shown in Figures 21-23. For the G141 spectroscopic light curve fits, the results were essentially identical to those presented in Evans et al. (2016), so we do not duplicate them here. The only difference for the latter is a wavelength-uniform shift of $R_{\mathrm{p}} / R_{\star}$ by 0.0007 , in line with the revised white light curve analysis, which gives $R_{\mathrm{p}} / R_{\star}=$ $0.1218 \pm 0.0004$ (Table 1), compared with the previous estimate of $R_{\mathrm{p}} / R_{\star}=0.1211 \pm 0.0003$ (Evans et al. 2016). ${ }^{20}$

As shown in Figure 10, we obtain means and standard deviations for the inferred $\beta$ values across spectroscopic channels of $1.05 \pm 0.07$ for the G430lv1 data set, $1.06 \pm 0.09$ for the G430Lv2 data set, $1.05 \pm 0.05$ for the G750L data set, and $1.02 \pm 0.06$ for the G141 data set. The consistency of these results with $\beta=1$ indicate that the GP models are broadly successful at marginalizing over the correlations in the light curves, implying in turn that degeneracies between the systematics and planet signal are properly accounted for in our estimates of parameters such as $R_{\mathrm{p}} / R_{\star}$, which we are primarily interested in.

To investigate the sensitivity of our results to the choice of covariance kernel, we repeated the spectroscopic light curve fitting using the Matérn $\nu=3 / 2$ kernel, which can be more suitable for modeling high-frequency signals than the squaredexponential kernel (e.g., see Gibson et al. 2012). For all channels, we found the inferred $R_{\mathrm{p}} / R_{\star}$ values remained unchanged to well within $1 \sigma$, regardless of which covariance kernel was used. However, the $\beta$ values inferred using the

\footnotetext{
20 The revised value for $R_{\mathrm{p}} / R_{\star}$ within the G141 bandpass can be attributed to the updated values for $a / R_{\star}$ and $b$ adopted in the present study (Section 3.1).
} 

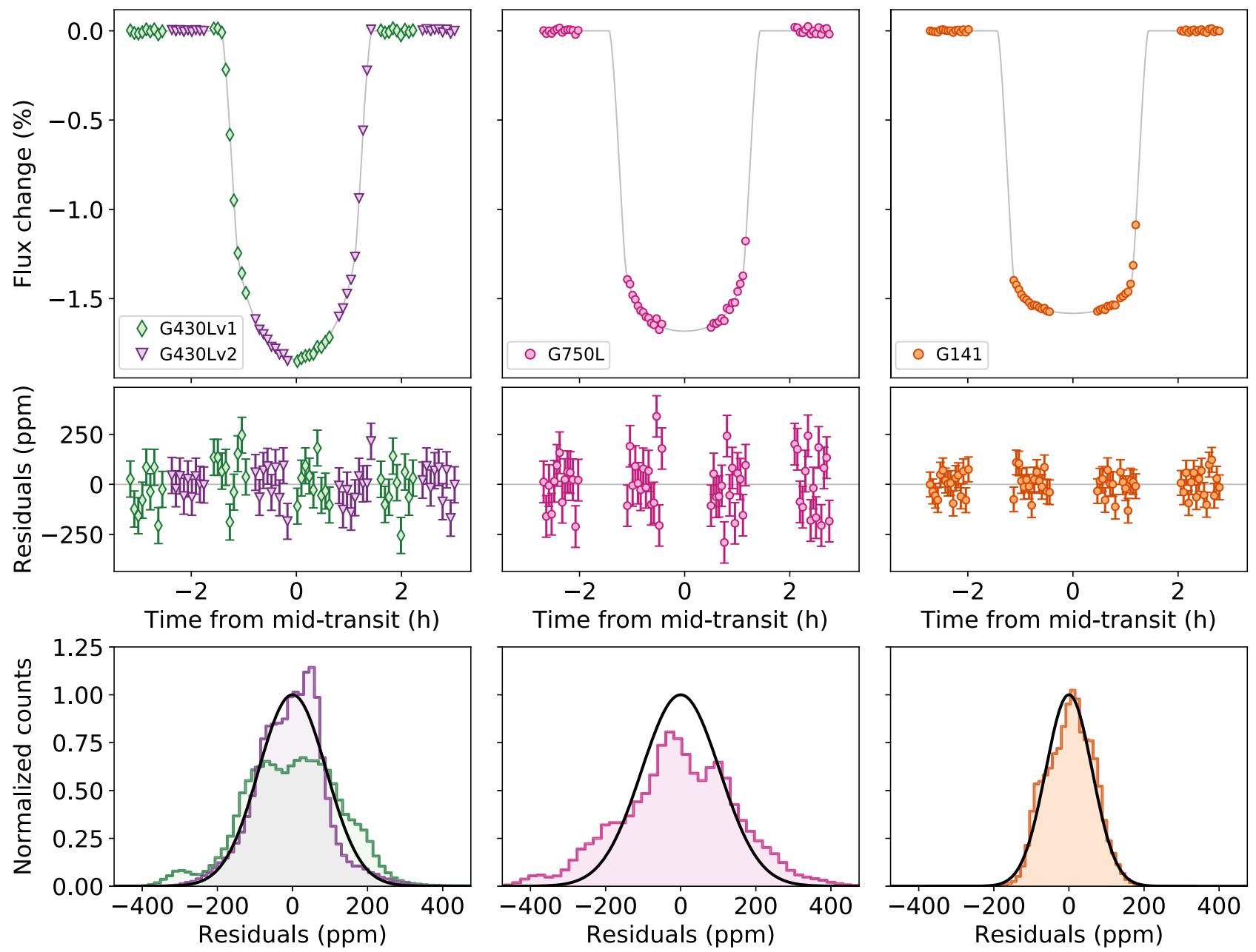

Time from mid-transit (h)

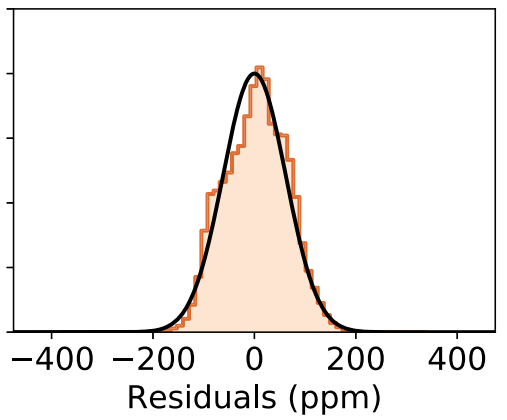

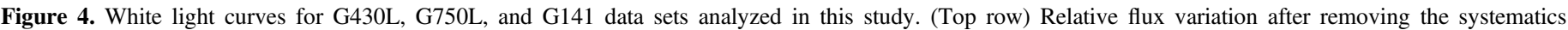

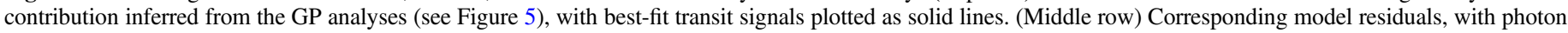

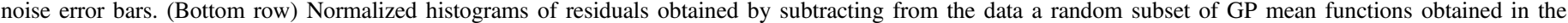

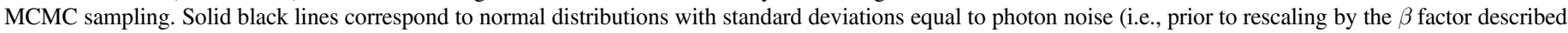
in the main text).

Matérn $\nu=3 / 2$ kernel were on average slightly closer to unity, as illustrated in Figure 10. This suggests that some of the channels may contain high-frequency noise that can be suitably accounted for either by inflating the white-noise level above the photon noise floor via $\beta>1$ or by employing a covariance kernel with enough flexibility to marginalize over signals of this nature, such as the Matérn $\nu=3 / 2$. Given that the results for $R_{\mathrm{p}} / R_{\star}$ are found to be insensitive to the choice of covariance kernel, we adopt those obtained using the squared exponential for the remainder of this paper.

The corresponding posterior distributions for $R_{\mathrm{p}} / R_{\star}, u_{1}$, and $u_{2}$ are summarized for each STIS data set in Tables 2 and 3. The median uncertainties on $R_{\mathrm{p}} / R_{\star}$ are $800 \mathrm{ppm}$ for G430L, $900 \mathrm{ppm}$ for G750L, and $500 \mathrm{ppm}$ for G141, which translate to uncertainties on the transit depth $\left(R_{\mathrm{p}} / R_{\star}\right)^{2}$ of approximately $200 \mathrm{ppm}, 220 \mathrm{ppm}$, and $125 \mathrm{ppm}$, respectively. For comparison, a change in the effective planetary radius of one atmospheric pressure scale height $H$ corresponds to a transit depth variation of $\sim 150-200 \mathrm{ppm}$ for WASP-121b, assuming average limb temperatures in the range of $1500-2000 \mathrm{~K}$, a planetary surface gravity of $940 \mathrm{~cm} \mathrm{~s}^{-2}$, and an atmospheric mean molecular weight of $\mu=2.22$ atomic mass units (i.e., equal to that of Jupiter).

\section{Discussion}

The measured transmission spectrum is shown in Figure 11 and has a number of notable features. In particular, the G430L data exhibit a steep rise toward shorter wavelengths from $\sim 0.47 \mu \mathrm{m}$, where $R_{\mathrm{p}} / R_{\star} \sim 0.121$, to $\sim 0.28 \mu \mathrm{m}$, where $R_{\mathrm{p}} / R_{\star} \sim 0.125$. This corresponds to a change in effective planetary radius of approximately five pressure scale heights. At the longer optical wavelengths covered by the G430L and G750L gratings $(\sim 0.47-1 \mu \mathrm{m}), R_{\mathrm{p}} / R_{\star}$ is measured to vary across spectroscopic channels, implying a wavelength-dependent atmospheric opacity. Within some of these optical channels, the atmospheric opacity is found to be even higher than that measured within the $\mathrm{H}_{2} \mathrm{O}$ band at $1.4 \mu \mathrm{m}$, which is detected in the G141 bandpass (Evans et al. 2016).

Figure 11 also shows the $R_{\mathrm{p}} / R_{\star}$ values measured using ground-based photometry in the $B, r^{\prime}$, and $z^{\prime}$ bandpasses. The latter were originally reported by Delrez et al. (2016), and an independent analysis of the same light curves was presented by Evans et al. (2016). Both studies obtained similar estimates for $R_{\mathrm{p}} / R_{\star}$ in each bandpass that are larger than those obtained from the HST data. It is unclear what is responsible for this tension. As noted above, updated values for $a / R_{\star}$ and $b$ were used in the light curve fits of the present study. However, for 

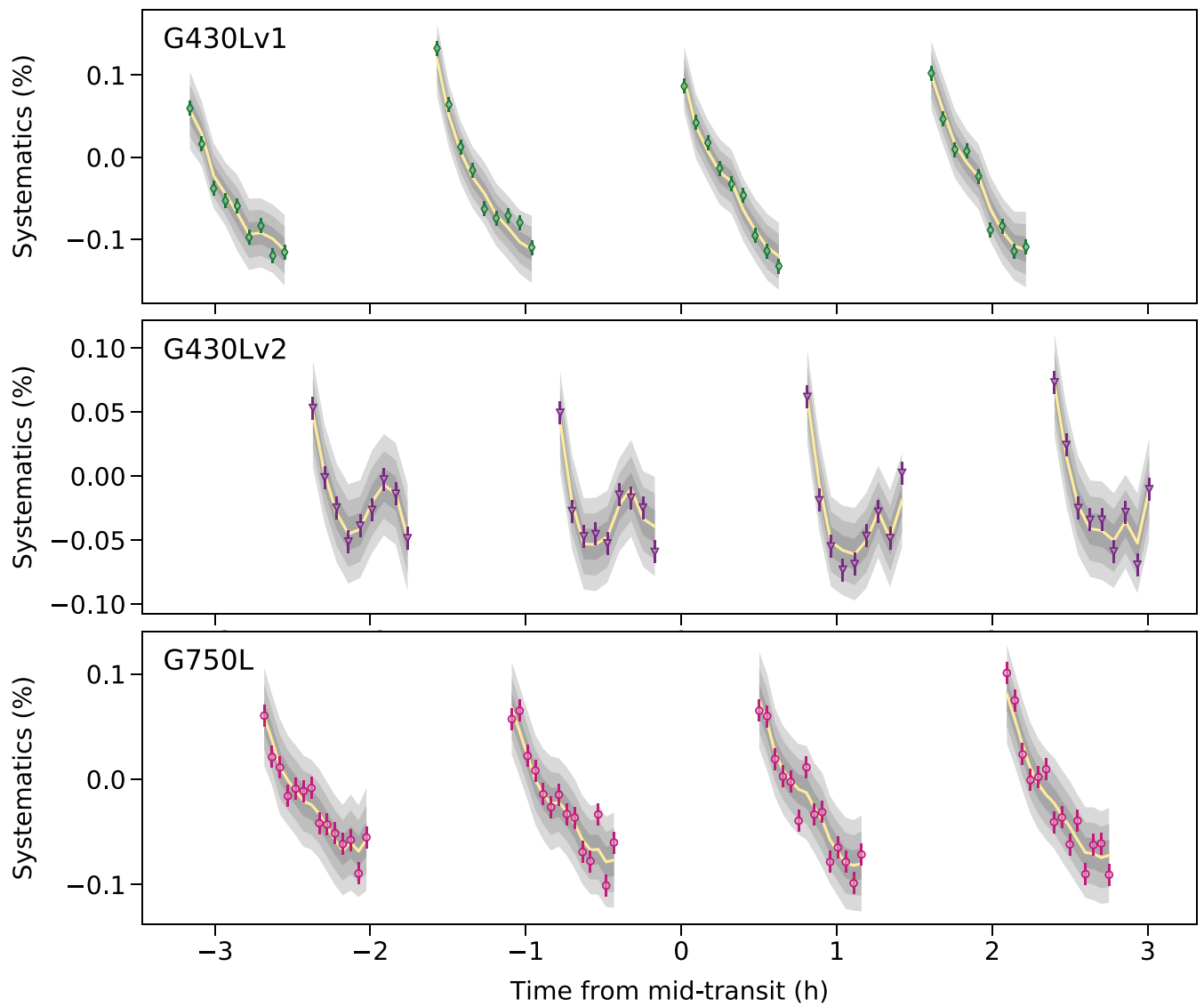

Figure 5. Systematics in the white light curves for the G430L and G750L data sets. Effectively, these are the residuals after dividing the raw flux time series by the transit signals with linear baseline trends shown in Figure 2. Yellow lines and gray shaded regions, respectively, show the means and $1 \sigma, 2 \sigma$, and $3 \sigma$ ranges of the bestfit GP distributions. Note that in practice the transit signal, linear baseline trend, and GP are fit simultaneously. The purpose of this figure is only to highlight the structure of the systematics.

the G141 data set, this had the effect of shifting the mean $R_{\mathrm{p}} / R_{\star}$ value to a higher value, from $0.1211 \pm 0.0003$ (Evans et al. 2016) to $0.1218 \pm 0.0004$ (Table 1). A similar upward shift for the ground-based photometry would make those data more discrepant relative to the $H S T$ data. Alternatively, during the photometry data reduction, effects such as aperture light losses or an overestimated background may have artificially deepened the transit signals, resulting in $R_{\mathrm{p}} / R_{\star}$ estimates above the true values. Another more speculative possibility is intrinsic variability of the atmosphere from epoch to epoch. For example, Parmentier et al. (2013) report a 3D GCM study showing that significant variations in passive tracer abundances over $\sim 100$ day timescales are possible at the planetary limb of hot Jupiters. As those authors note, if this occurs for strongly absorbing species such as $\mathrm{TiO}$ and $\mathrm{VO}$, it could have significant implications for transmission spectra measured at different epochs. Indeed, the ground-based photometry and HST STIS observations were separated by over 100 days. However, the current data are insufficient to test this theory, and we consider it more likely that the difference is due to some unaccounted-for systematic in the ground-based photometry.

To evaluate the robustness of the HST transmission spectrum, we performed a number of tests, full details of which are reported in Appendix B. First, we find that the measured transmission spectrum is insensitive to our treatment of limb darkening. Second, we investigated the inclusion of time $t$ as an additional GP input variable in the light curve fits and obtain very similar results to those reported here. Third, for the G430L data, we find that the measured transmission spectrum is repeatable when each of the two visits are analyzed separately. Fourth, we conclude that stellar activity is unlikely to have significantly affected the measured transmission spectrum, based on (1) the lack of photometric variability and modest X-ray flux of the WASP-121 host star, (2) the epoch-to-epoch repeatability of the G430L data sets, (3) the good level of agreement obtained across the overlapping wavelength range of the G430L and G750L data sets, and (4) the inability of unocculted spots to explain the shape of the measured spectrum under reasonable assumptions. In the following sections, we therefore seek to interpret the measurements shown in Figure 11 as the signal of the planetary atmosphere.

\subsection{Rayleigh Scattering and a Gray Cloud Deck}

The signature of aerosol scattering is ubiquitous in observations of exoplanet atmospheres (e.g., Pont et al. 2008; Kreidberg et al. 2014; Nikolov et al. 2014, 2015; Sing et al. 2015). For hot Jupiter transmission spectra, this is unsurprising given the large number of refractory species expected to condense at the temperatures and pressures characteristic of these atmospheres (e.g., Woitke et al. 2018), as well as the highly sensitive nature of the grazing geometry to even trace opacity sources (Fortney 2005). Indeed, the rise in opacity toward shorter wavelengths that we measure for WASP-121b is somewhat reminiscent of transmission spectra previously 


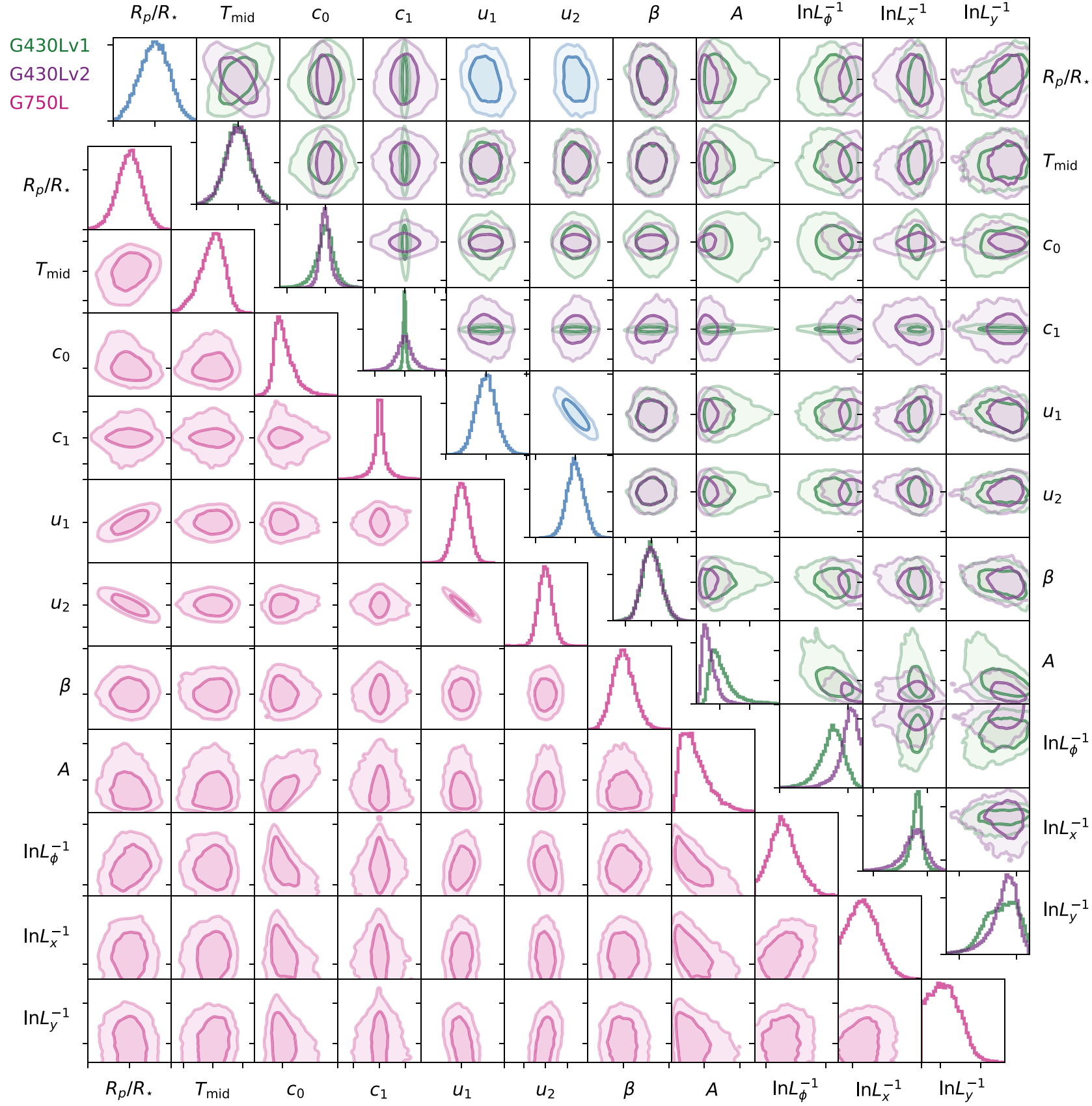

Figure 6. Posterior distributions obtained from the white light curve analyses described in Section 3.2. The top-right panels show results for the joint analysis of both G430L visits, and the bottom-left panels show results for the G750L analysis. Plotted contours contain $68 \%$ and $95 \%$ of the MCMC samples. Panels along the diagonal show the marginalized posterior distributions. Note that $T_{\mathrm{mid}}, c_{0}, c_{1}$, and $\beta$ have been median-subtracted to allow both $\mathrm{G} 430 \mathrm{~L}$ visits to be plotted on the same axes. The purpose of this figure is to visually illustrate correlations between model parameters. Numerical values for all parameter distributions are summarized in Table 1.

obtained for other hot Jupiters, which can be explained by Rayleigh scattering due to high-altitude layers of submicron aerosols (Sing et al. 2016). In addition, an optically thick cloud deck could act as a gray opacity source, if present at low pressures.

We investigated how well the WASP-121b transmission spectrum can be explained by aerosols by first fitting simple Rayleigh scattering and cloud deck models to the STIS data spanning the G430L and G750L gratings. For the Rayleigh component, we followed the methodology outlined by Lecavelier Des Etangs et al. (2008, hereafter L08), who provide relations between the slope of the transmission spectrum and the atmospheric temperature, under the assumption of scattering particles distributed uniformly with pressure. For the cloud deck component, we assumed a wavelengthindependent opacity, implemented as a horizontal flat line in $R_{\mathrm{p}} / R_{\star}$ that was allowed to float vertically relative to other spectral features in the transmission spectrum. For this initial analysis, we excluded the G141 data set, as it exhibits a clear spectral feature due to $\mathrm{H}_{2} \mathrm{O}$, which would add additional complexity to the model. This is addressed in Section 5.4, where we perform a free-chemistry fit to the combined STIS +WFC3 data set that includes opacity due to both gas-phase species and aerosols. 

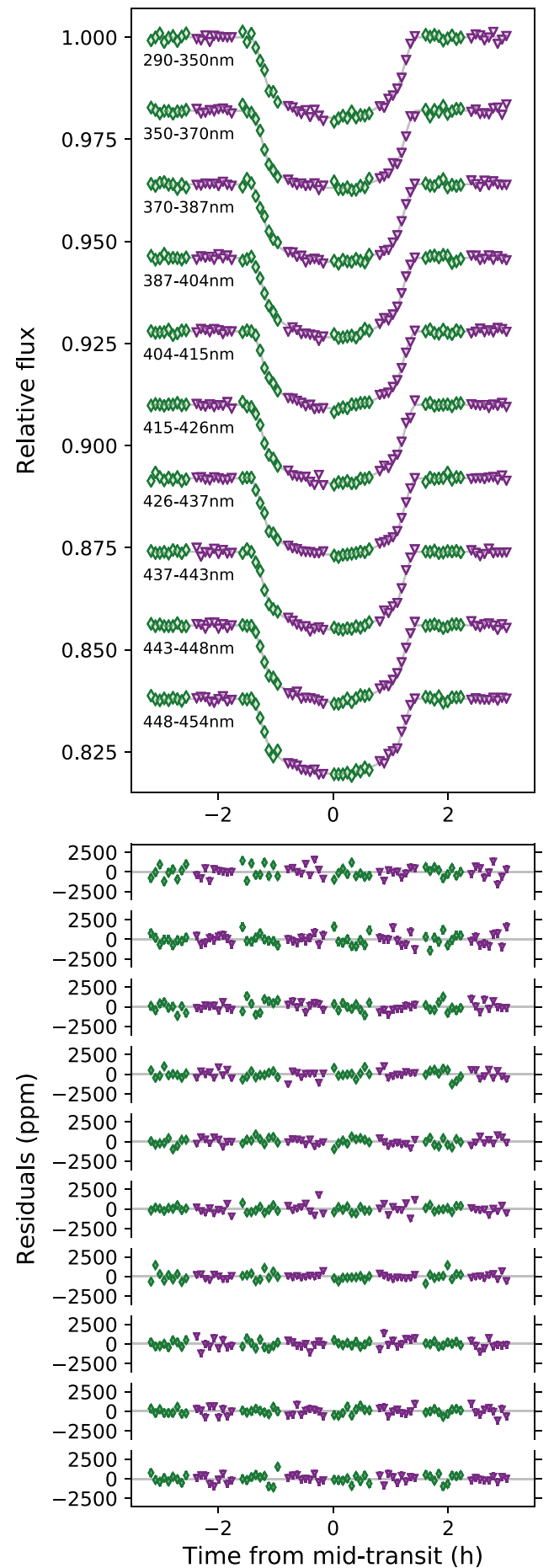

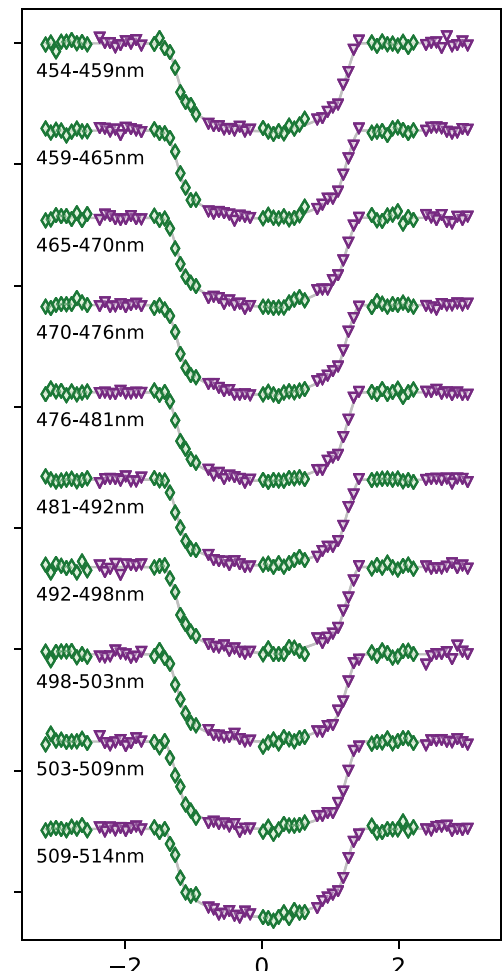

$-2$
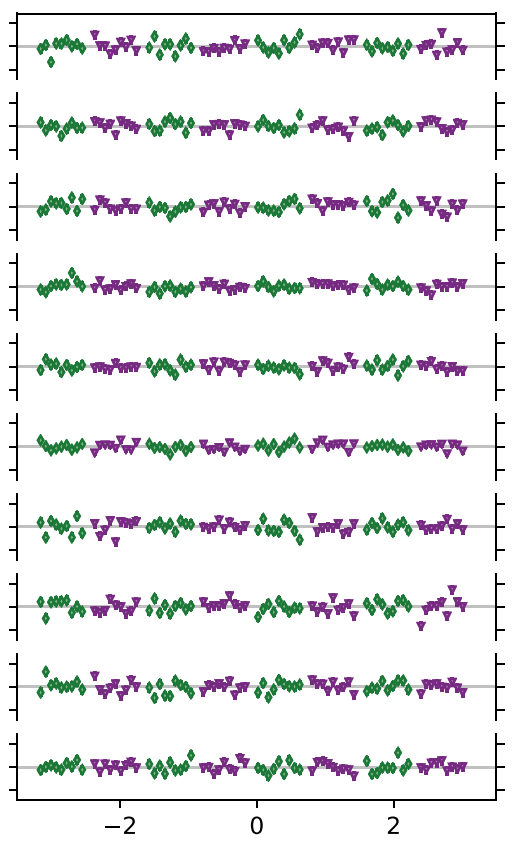

Time from mid-transit (h)
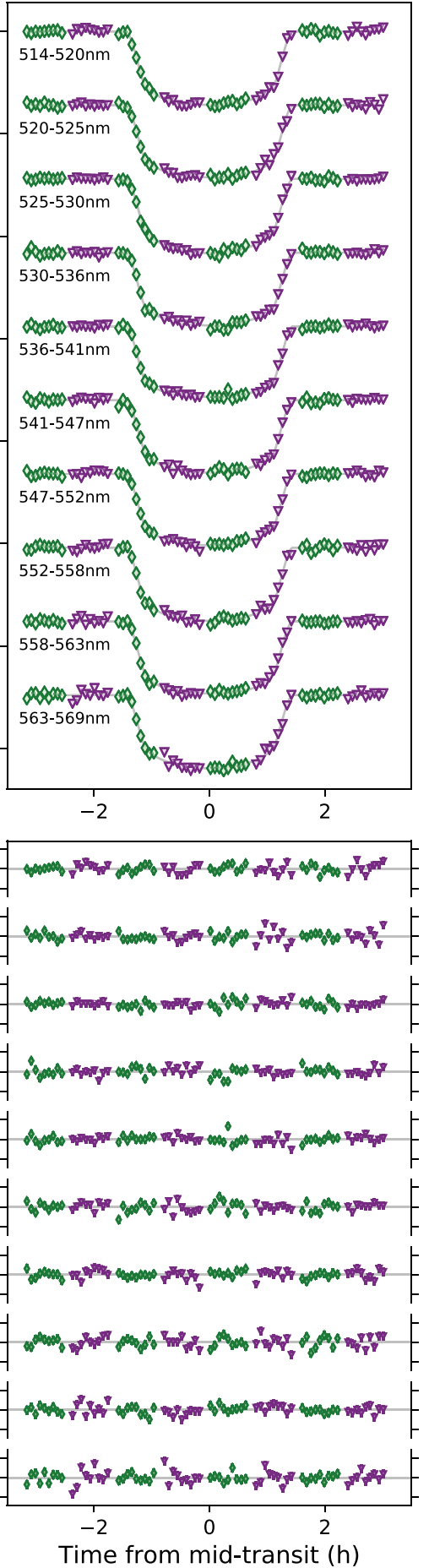

Figure 7. Spectroscopic light curves for the G430Lv1 and G430Lv2 data sets after removing the systematics contributions inferred from the GP analyses, with best-fit transit signals plotted as solid lines. The green triangles and purple diamonds correspond to the G430Lv1 and G430Lv2 data sets, respectively.

Our best-fit model combining a Rayleigh slope with a cloud deck is shown in Figure 12. It provides a poor fit to the data, with a reduced $\chi^{2}$ of 1.8 for 57 degrees of freedom, allowing us to exclude it at $3.7 \sigma$ confidence. This is due to the inability of a featureless cloud deck to explain the optical data across the $0.47-1 \mu \mathrm{m}$ wavelength range. Furthermore, the temperature inferred from the Rayleigh slope is $6980 \pm 3660 \mathrm{~K}$, which is improbably high for the atmospheric pressures probed in transmission. For instance, if WASP-121b absorbs all incident radiation on its dayside hemisphere (i.e., the Bond albedo is zero), then the substellar point would have a temperature of $T_{\star} / \sqrt{a / R_{\star}} \sim 3280 \mathrm{~K}$, and the day-night boundary probed by the transmission spectrum should be considerably cooler. Furthermore, at such high temperatures, no condensates are expected to exist, and molecules should be thermally dissociated, including $\mathrm{H}_{2}$.

To be conservative, we also tried dividing the NUV-optical data into different wavelength sections and fitting them one at a time. For convenience, we will refer to these subsets as the blue data $(0.3-0.47 \mu \mathrm{m})$ and the red data $(0.47-1 \mu \mathrm{m})$. In principle, 

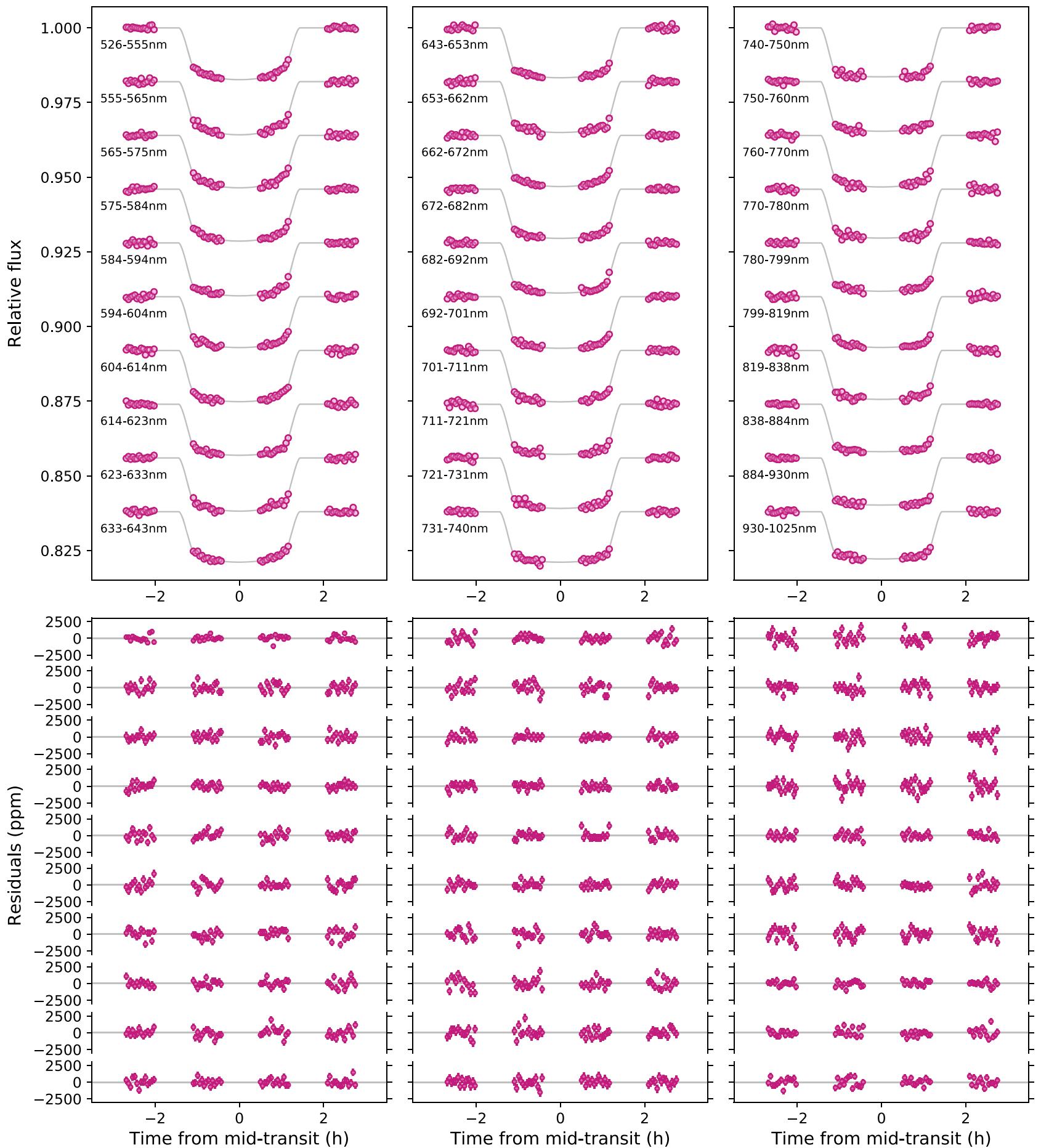

Figure 8. Similar to Figure 7, but for the G750L spectroscopic light curves.

a good fit to one or both of these data sets separately should be easier to achieve than a good joint fit, as the models need not be self-consistent.

First, we fit a Rayleigh profile to the blue data, as this is where the transmission spectrum exhibits a strong slope. Although we obtain a better statistical fit with a reduced $\chi^{2}$ of 1.6 for 10 degrees of freedom (Figure 12), the inferred temperature remains implausibly high at $6810 \pm 1530 \mathrm{~K}$. Given this, we conclude that the rise in the measured transmission spectrum toward NUV wavelengths is too steep to be explained by scattering out of the transmission beam. Instead, it would suggest the presence of one or more significant NUV absorbers in the upper atmosphere of WASP-121b, assuming the slope is indeed a feature of the planetary spectrum and not caused by an uncorrected systematic effect in the data.

Second, we tried fitting a gray cloud deck to the red data. For this scenario (not shown in Figure 12), we obtain a reduced $\chi^{2}$ of 1.9 for 51 degrees of freedom, which formally rules it out at $3.8 \sigma$ confidence. Alternatively, if the $R_{\mathrm{p}} / R_{\star}$ uncertainties for 


\begin{tabular}{|c|c|c|c|c|c|c|c|c|c|c|c|c|}
\hline & & & & & & G4 & & & & & & \\
\hline $\begin{array}{r}0.25 \\
0.00 \\
-0.25\end{array}$ & $\dot{\Delta} \vec{\Delta}$ & 然o & Aa & ày & 5 & 离 & $\vec{\nabla}$ & 者 & $\infty$ & 8 & 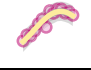 & 8 \\
\hline $\begin{array}{r}0.25 \\
0.00 \\
-0.25\end{array}$ & $\frac{a-m}{\mathrm{mw}}$ & $\frac{\Delta-1}{m-\infty}$ & 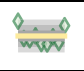 & 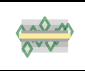 & 语 & ज्ञ & 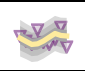 & 徣 & $\cos$ & 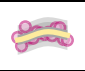 & $\infty$ & ळबके \\
\hline $\begin{array}{r}0.25 \\
0.00 \\
-0.25\end{array}$ & 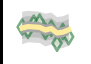 & and & 슴 & 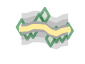 & 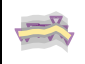 & 整育 & 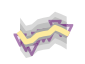 & $\sqrt{5}$ & $\approx$ & $\approx$ & \&s & $\approx$ \\
\hline & $\frac{a \Delta n}{w m}$ & 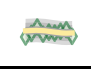 & $\vec{m} \Rightarrow$ & ma & $\frac{-m b}{m-n}$ & $\stackrel{\nabla}{\nabla}$ & $\stackrel{x \rightarrow}{m}$ & mom & 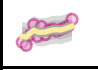 & cos & $\approx$ & \&s \\
\hline $\begin{array}{r}0.25 \\
0.00 \\
-0.25\end{array}$ & 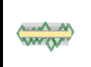 & $\min$ & arm & Anm & 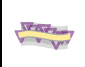 & $\overrightarrow{\mathrm{m}}$ & aㅔ & $\stackrel{x \rightarrow m}{\Rightarrow}$ & అు⿻ & $\infty$ & $\operatorname{lom}_{0}$ & $\varpi$ \\
\hline $\begin{array}{r}0.25 \\
0.00 \\
-0.25\end{array}$ & 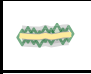 & ind & $\stackrel{n m}{n m}$ & 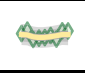 & 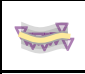 & $\frac{R=0}{N}$ & $\stackrel{x \rightarrow 0}{0}$ & 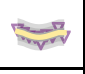 & tand & $\Leftrightarrow$ & 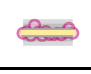 & बिज्ञ \\
\hline $\begin{array}{r}0.25 \\
0.00 \\
-0.25\end{array}$ & 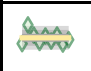 & $\stackrel{m \Delta x}{m}$ & $\Longrightarrow$ & 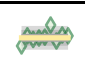 & thesy & 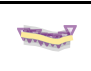 & 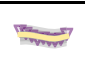 & ${ }^{\infty}$ & ख్య० & nas & $\infty$ & कून \\
\hline $\begin{array}{r}0.25 \\
0.00 \\
-0.25\end{array}$ & $\widehat{D A \Delta}$ & $\stackrel{\sim}{\infty}$ & $\forall$ & $\Leftrightarrow$ & 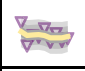 & 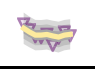 & Zntw & 称 & $\stackrel{\infty}{\infty}$ & $\Leftrightarrow$ & 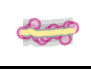 & cos \\
\hline & 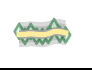 & $\Rightarrow$ & $\stackrel{\Delta a d y}{w a v e}$ & ma & 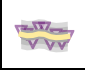 & $\stackrel{\square}{\infty}$ & ins & $\frac{\mathrm{Ve}}{\mathrm{w}^{\prime}}$ & 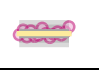 & बूले & $\frac{2 \pi}{20}$ & 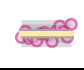 \\
\hline $\begin{array}{r}0.25 \\
0.00 \\
-0.25\end{array}$ & ind & $-\infty$ & $\sin _{\text {now }}$ & $\frac{\mathrm{m} M \mathrm{~m}}{\mathrm{vov}}$ & 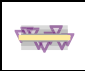 & 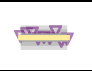 & 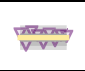 & $\stackrel{m}{m}$ & 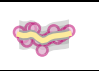 & ins & సీతి & $\approx$ \\
\hline $\begin{array}{r}0.25 \\
0.00 \\
-0.25\end{array}$ & $\Rightarrow$ & 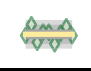 & 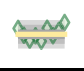 & 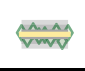 & $\mathrm{Z}_{\mathrm{m}}$ & $\sqrt{1 . m}$ & mas & $\frac{\nabla}{\nabla-m}$ & 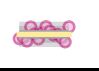 & 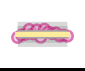 & 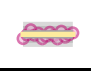 & $\infty$ \\
\hline $\begin{array}{r}0.25 \\
0.00 \\
-0.25\end{array}$ & 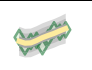 & 然 & $\infty$ & $\infty$ & $\stackrel{m}{=1}$ & $\sqrt{w \mathrm{v}}$ & $\stackrel{B \rightarrow}{m}$ & 些- & ind & aㅏ & हित् & Doss \\
\hline $\begin{array}{r}0.25 \\
0.00 \\
-0.25\end{array}$ & $\operatorname{mas}_{m \infty}$ & Dr. & was & $\frac{\sin 2}{w-\pi}$ & 要票 & $\stackrel{n}{n}$ & Tse & 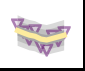 & జ & $\approx$ & $\Leftrightarrow$ & होक्षे \\
\hline $\begin{array}{r}0.25 \\
0.00 \\
-0.25\end{array}$ & $\stackrel{g}{\Longleftrightarrow}$ & क्राप & mam & $\stackrel{m a}{m}$ & 믈 & 맥 & $\stackrel{m}{\Rightarrow}$ & $m$ & sos & $\infty$ & $\approx 3$ & cos \\
\hline $\begin{array}{r}0.25 \\
0.00 \\
-0.25\end{array}$ & $m$ & $m_{\infty}$ & $n=$ & $\sin _{0}$ & 들 & $\sum^{2}=$ & the & $8=$ & \&s & $\infty$ & 20 & $\approx$ \\
\hline & ther & $\operatorname{mim}_{\operatorname{man}}$ & $\operatorname{man}$ & 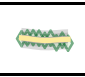 & $\stackrel{m}{m}$ & 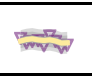 & $\stackrel{m}{\square}$ & $\overline{\nabla v}$ & कीज़ & 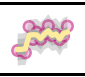 & 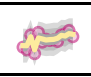 & mom \\
\hline & sind & $\stackrel{n a}{n}$ & 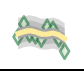 & $\stackrel{m}{m}$ & 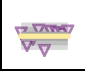 & $\stackrel{-m}{m}$ & $\stackrel{\mathrm{r}}{-m}$ & 点 & $\lim _{\infty}$ & $\cong$ & $\stackrel{\infty}{\infty}$ & $\stackrel{m}{m}$ \\
\hline $\begin{array}{r}0.25 \\
0.00 \\
-0.25\end{array}$ & 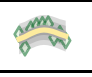 & 票 & 整 & 亚 & $\frac{\vec{m}}{w-w}$ & $\stackrel{\Delta}{\Longrightarrow}$ & $\frac{\nabla-}{w m}$ & $\frac{\vec{\nabla}}{\vec{v} v}$ & $\frac{\infty}{\infty+\infty}$ & Dam & क्जि & sing \\
\hline $\begin{array}{r}0.25 \\
0.00 \\
-0.25\end{array}$ & $\stackrel{\Delta m}{\Rightarrow}$ & $0 \times$ & 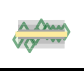 & $\stackrel{\mathrm{m}}{\mathrm{m}}$ & $\frac{T-2}{w v E}$ & $\overrightarrow{v \vec{v}}$ & $\sin$ & 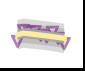 & $\stackrel{-0}{=0}$ & $\stackrel{\circ}{\approx}$ & $\cos _{0}$ & $\approx$ \\
\hline $\begin{array}{r}0.25 \\
0.00 \\
-0.25\end{array}$ & $\Leftrightarrow$ & $\overrightarrow{\mathrm{W} w \mathrm{C}}$ & बraw & $\stackrel{\Delta}{\vec{w}} \overrightarrow{\mathrm{m}}$ & 霄 & tor & 贾言 & $\stackrel{n}{n}$ & cimet & हिकी & coss & $\mathbb{1}_{0}$ \\
\hline $\begin{array}{r}0.25 \\
0.00 \\
-0.25\end{array}$ & 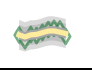 & 祭 & $\min _{w \infty}$ & mand & 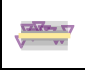 & 要要 & $\frac{\nabla D}{n=0}$ & $\frac{\vec{v}}{\overrightarrow{v v}}$ & $\overbrace{\infty \infty}^{\infty}$ & कीजी & है-men & ज्ञज्ञ \\
\hline $\begin{array}{r}0.25 \\
0.00 \\
-0.25\end{array}$ & and & 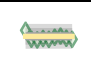 & 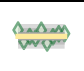 & $\stackrel{m a n}{m}$ & $\underline{E-m}$ & $\stackrel{a-b}{m=3}$ & $\frac{\nabla \nabla_{n}}{\nabla \cdots \nabla}$ & $\frac{D-D V}{\nabla V D}$ & fos & का & $\sum_{0}^{\infty}$ & लs: \\
\hline $\begin{array}{r}0.25 \\
0.00 \\
-0.25\end{array}$ & $\stackrel{m}{m}$ & 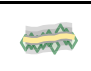 & 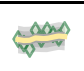 & $\frac{m}{m-x}$ & $\Longrightarrow$ & 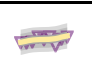 & 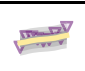 & 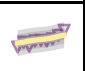 & ${ }_{2}$ & $S_{\infty}$ & 23 & $C_{0.0}^{\infty}$ \\
\hline $\begin{array}{r}0.25 \\
0.00 \\
-0.25\end{array}$ & \& & $\frac{\operatorname{man}}{c}$ & $=$ & $\stackrel{s=n}{n}$ & $\approx$ & 마메 & Drm & - & 피 & ges & $\infty_{0}$ & $\frac{a n}{2 n d}$ \\
\hline $\begin{array}{r}0.25 \\
0.00 \\
-0.25\end{array}$ & $\hat{\sim}$ & and & $e_{0}$ & 슬 & 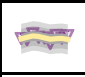 & 量 & $\overrightarrow{w_{b}}$ & 글 & $\approx$ & Ci:o & $\hat{s}$ & 32 \\
\hline $\begin{array}{r}0.25 \\
0.00 \\
-0.25\end{array}$ & $\frac{\Delta \max }{w}$ & 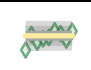 & $\Delta$ & $\stackrel{\Delta M}{\Delta M}$ & vis & 点 & 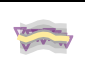 & $\approx$ & Ser & Sos & $\approx$ & $\mathrm{aes}_{3}$ \\
\hline $\begin{array}{r}0.25 \\
0.00 \\
-0.25\end{array}$ & $\stackrel{s m}{w \infty}$ & 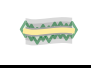 & maxe & 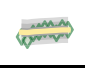 & mam & $\sqrt{=7}$ & $\overrightarrow{v m}$ & 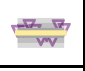 & कam & 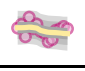 & $\Leftrightarrow$ & mas \\
\hline $\begin{array}{r}0.25 \\
0.00 \\
-0.25\end{array}$ & $\underset{m \infty}{m}$ & $\min _{\rightarrow \infty}$ & $\frac{m=}{a m}$ & $\Delta \Delta$ & $\stackrel{D-m}{w-1}$ & ㅁon & $\frac{\nabla=0}{m \square}$ & $\frac{\mathrm{max}}{\mathrm{NWW}}$ & $\Leftrightarrow$ & क्s & $\rightarrow$ & sos \\
\hline $\begin{array}{r}0.25 \\
0.00 \\
-0.25\end{array}$ & Ama & $\overrightarrow{n ल}$ & 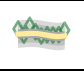 & $\Rightarrow$ & 登 & 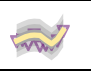 & 照 & $\mathbb{v}$ & $\approx \approx$ & bages & 10 & nas \\
\hline $\begin{array}{r}0.25 \\
0.00 \\
-0.25\end{array}$ & and & $\approx$ & $\Rightarrow$ & 매 & $\frac{\nabla \nabla}{\nabla}$ & $\frac{\nabla_{\mathrm{g}}}{\mathrm{vav}}$ & $\frac{k}{v \nabla}$ & $\frac{\nabla m}{b \rightarrow}$ & gess & atos & 8 & \\
\hline & & from & $\begin{array}{l}0 \\
\text { d-tran }\end{array}$ & 2 & & $\begin{array}{r}0 \\
\text { from } n\end{array}$ & d-trans & & $\begin{array}{l}-2 \\
\text { Tim }\end{array}$ & from & d-trans & \\
\hline
\end{tabular}

Figure 9. Similar to Figure 5, but showing the systematics and GP fits for the spectroscopic light curves. In all columns, wavelength increases from top to bottom. 
Table 2

Results of the G430L Spectroscopic Light Curve Fits for Selected Parameters

\begin{tabular}{|c|c|c|c|c|c|c|c|}
\hline$\lambda(\AA)$ & $R_{\mathrm{p}} / R_{\star}$ & $u_{1}$ & $u_{2}$ & $\beta_{v 1}$ & $\sigma_{v 1}(\mathrm{ppm})$ & $\beta_{v 2}$ & $\overline{\sigma_{v 2}(\mathrm{ppm})}$ \\
\hline $2898-3499$ & $0.1246_{-0.0011}^{+0.0010}$ & $0.55_{-0.08}^{+0.08}$ & $0.21_{-0.13}^{+0.13}$ & $1.16_{-0.10}^{+0.10}$ & $565_{-49}^{+47}$ & $1.15_{-0.11}^{+0.10}$ & $557_{-52}^{+50}$ \\
\hline $3499-3700$ & $0.1238_{-0.0010}^{+0.0009}$ & $0.41_{-0.09}^{+0.09}$ & $0.30_{-0.14}^{+0.14}$ & $1.03_{-0.09}^{+0.11}$ & $593_{-54}^{+61}$ & $1.10_{-0.09}^{+0.10}$ & $635_{-51}^{+55}$ \\
\hline $3700-3868$ & $0.1235_{-0.0011}^{+0.0012}$ & $0.41_{-0.10}^{+0.10}$ & $0.39_{-0.15}^{+0.14}$ & $1.11_{-0.11}^{+0.11}$ & $550_{-54}^{+56}$ & $1.13_{-0.11}^{+0.11}$ & $559_{-53}^{+52}$ \\
\hline $3868-4041$ & $0.1223_{-0.0008}^{+0.0008}$ & $0.58_{-0.08}^{+0.08}$ & $0.23_{-0.12}^{+0.12}$ & $1.13_{-0.09}^{+0.09}$ & $439_{-36}^{+36}$ & $1.13_{-0.10}^{+0.10}$ & $440_{-37}^{+39}$ \\
\hline $4041-4151$ & $0.1211_{-0.0007}^{+0.0006}$ & $0.60_{-0.07}^{+0.07}$ & $0.15_{-0.11}^{+0.12}$ & $1.06_{-0.08}^{+0.09}$ & $438_{-34}^{+38}$ & $1.02_{-0.09}^{+0.10}$ & $422_{-36}^{+41}$ \\
\hline $4151-4261$ & $0.1227_{-0.0007}^{+0.0007}$ & $0.62_{-0.07}^{+0.07}$ & $0.09_{-0.12}^{+0.12}$ & $1.02_{-0.09}^{+0.10}$ & $399_{-36}^{+37}$ & $1.06_{-0.12}^{+0.12}$ & $413_{-47}^{+46}$ \\
\hline $4261-4371$ & $0.1230_{-0.0006}^{+0.0006}$ & $0.50_{-0.08}^{+0.08}$ & $0.14_{-0.12}^{+0.12}$ & $1.15_{-0.09}^{+0.10}$ & $452_{-35}^{+38}$ & $0.93_{-0.10}^{+0.11}$ & $365_{-40}^{+42}$ \\
\hline $4371-4426$ & $0.1225_{-0.0009}^{+0.0009}$ & $0.54_{-0.09}^{+0.08}$ & $0.21_{-0.13}^{+0.13}$ & $0.98_{-0.10}^{+0.11}$ & $504_{-52}^{+56}$ & $1.09_{-0.09}^{+0.09}$ & $560_{-46}^{+48}$ \\
\hline $4426-4481$ & $0.1209_{-0.0007}^{+0.0008}$ & $0.62_{-0.08}^{+0.08}$ & $0.08_{-0.13}^{+0.12}$ & $0.92_{-0.10}^{+0.10}$ & $463_{-51}^{+50}$ & $1.09_{-0.09}^{+0.09}$ & $551_{-47}^{+46}$ \\
\hline $4481-4536$ & $0.1196_{-0.0007}^{+0.0007}$ & $0.58_{-0.08}^{+0.07}$ & $0.18_{-0.12}^{+0.12}$ & $1.09_{-0.08}^{+0.09}$ & $539_{-41}^{+45}$ & $0.96_{-0.09}^{+0.10}$ & $473_{-45}^{+47}$ \\
\hline $4536-4591$ & $0.1208_{-0.0007}^{+0.0008}$ & $0.48_{-0.08}^{+0.08}$ & $0.21_{-0.13}^{+0.13}$ & $1.11_{-0.09}^{+0.09}$ & $554_{-44}^{+45}$ & $1.07_{-0.09}^{+0.09}$ & $531_{-44}^{+45}$ \\
\hline $4591-4646$ & $0.1211_{-0.0008}^{+0.0007}$ & $0.43_{-0.08}^{+0.07}$ & $0.29_{-0.12}^{+0.12}$ & $1.03_{-0.10}^{+0.10}$ & $507_{-50}^{+50}$ & $1.00_{-0.09}^{+0.10}$ & $493_{-44}^{+48}$ \\
\hline $4646-4701$ & $0.1205_{-0.0009}^{+0.0010}$ & $\begin{array}{r}0.55_{-0.09}^{+0.09} \\
0\end{array}$ & $0.14_{-0.14}^{+0.14}$ & $1.09_{-0.10}^{+0.10}$ & $546_{-48}^{+52}$ & $1.03_{-0.11}^{+0.10}$ & $517_{-53}^{+52}$ \\
\hline $4701-4756$ & $0.1224_{-0.0006}^{+0.0007}$ & $0.52_{-0.08}^{+0.08}$ & $0.11_{-0.12}^{+0.12}$ & $0.98_{-0.11}^{+0.10}$ & $492_{-54}^{+50}$ & $0.86_{-0.10}^{+0.10}$ & $434_{-50}^{+53}$ \\
\hline $4756-4811$ & $0.1216_{-0.0007}^{+0.0007}$ & $0.45_{-0.08}^{+0.08}$ & $0.24_{-0.12}^{+0.12}$ & $0.99_{-0.09}^{+0.09}$ & $498_{-46}^{+44}$ & $0.94_{-0.09}^{+0.09}$ & $471_{-45}^{+47}$ \\
\hline $4811-4921$ & $0.1214_{-0.0006}^{+0.0006}$ & $0.45_{-0.08}^{+0.08}$ & $0.10_{-0.12}^{+0.12}$ & $1.00_{-0.10}^{+0.10}$ & $373_{-36}^{+36}$ & $1.06_{-0.10}^{+0.10}$ & $395_{-38}^{+38}$ \\
\hline $4921-4976$ & $0.1208_{-0.0008}^{+0.0008}$ & $0.43_{-0.08}^{+0.08}$ & $0.21_{-0.13}^{+0.12}$ & $1.10_{-0.10}^{+0.10}$ & $557_{-48}^{+48}$ & $1.04_{-0.09}^{+0.10}$ & $522_{-46}^{+50}$ \\
\hline $4976-5030$ & $0.1216_{-0.0009}^{+0.0008}$ & $0.45_{-0.09}^{+0.09}$ & $0.20_{-0.14}^{+0.14}$ & $1.06_{-0.09}^{+0.09}$ & $539_{-46}^{+48}$ & $1.11_{-0.09}^{+0.09}$ & $562_{-46}^{+46}$ \\
\hline $5030-5085$ & $0.1230_{-0.0008}^{+0.0008}$ & $0.40_{-0.08}^{+0.08}$ & $0.16_{-0.13}^{+0.13}$ & $1.08_{-0.08}^{+0.09}$ & $542_{-41}^{+48}$ & $1.03_{-0.09}^{+0.10}$ & $516_{-47}^{+50}$ \\
\hline $5085-5140$ & $0.1222_{-0.0008}^{+0.0007}$ & $0.50_{-0.09}^{+0.08}$ & $0.07_{-0.13}^{+0.14}$ & $1.06_{-0.09}^{+0.09}$ & $531_{-45}^{+47}$ & $1.01_{-0.10}^{+0.10}$ & $507_{-52}^{+51}$ \\
\hline $5140-5195$ & $0.1234_{-0.0008}^{+0.0008}$ & $0.36_{-0.09}^{+0.09}$ & $0.20_{-0.13}^{+0.13}$ & $0.99_{-0.10}^{+0.10}$ & $511_{-51}^{+54}$ & $\begin{array}{l}1.09_{-0.08}^{+0.09} \\
-.09\end{array}$ & $561_{-43}^{+49}$ \\
\hline $5195-5250$ & $0.1224_{-0.0008}^{+0.0008}$ & $0.36_{-0.08}^{+0.08}$ & $0.18_{-0.13}^{+0.13}$ & $0.95_{-0.10}^{+0.10}$ & $486_{-49}^{+54}$ & $1.15_{-0.09}^{+0.10}$ & $592_{-46}^{+49}$ \\
\hline $5250-5305$ & $0.1215_{-0.0007}^{+0.0007}$ & $0.48_{-0.09}^{+0.08}$ & $0.17_{-0.13}^{+0.13}$ & $1.02_{-0.09}^{+0.09}$ & $529_{-46}^{+45}$ & $0.89_{-0.10}^{+0.10}$ & $462_{-52}^{+51}$ \\
\hline $5305-5360$ & $0.1223_{-0.0009}^{+0.0009}$ & $0.38_{-0.09}^{+0.09}$ & $0.16_{-0.14}^{+0.13}$ & $1.10_{-0.09}^{+0.10}$ & $572_{-46}^{+54}$ & $1.05_{-0.09}^{+0.10}$ & $545_{-48}^{+52}$ \\
\hline $5360-5415$ & $0.1221_{-0.0010}^{+0.0009}$ & $0.27_{-0.09}^{+0.09}$ & $0.27_{-0.13}^{+0.14}$ & $1.06_{-0.10}^{+0.11}$ & $559_{-53}^{+59}$ & $0.99_{-0.10}^{+0.10}$ & $523_{-52}^{+53}$ \\
\hline $5415-5469$ & $0.1218_{-0.0008}^{+0.0009}$ & $0.34_{-0.09}^{+0.08}$ & $0.30_{-0.13}^{+0.13}$ & $1.07_{-0.09}^{+0.10}$ & $573_{-48}^{+52}$ & $1.04_{-0.10}^{+0.10}$ & $553_{-52}^{+52}$ \\
\hline $5469-5524$ & $0.1210_{-0.0008}^{+0.0007}$ & $0.38_{-0.08}^{+0.08}$ & $0.21_{-0.13}^{+0.13}$ & $0.93_{-0.09}^{+0.11}$ & $502_{-51}^{+57}$ & $1.06_{-0.09}^{+0.10}$ & $571_{-48}^{+54}$ \\
\hline $5524-5579$ & $0.1229_{-0.0008}^{+0.0008}$ & $0.40_{-0.09}^{+0.08}$ & $0.14_{-0.13}^{+0.14}$ & $1.06_{-0.09}^{+0.09}$ & $573_{-47}^{+48}$ & $1.13_{-0.09}^{+0.09}$ & $613_{-48}^{+50}$ \\
\hline $5579-5634$ & $0.1219_{-0.0009}^{+0.0008}$ & $0.28_{-0.09}^{+0.09}$ & $0.36_{-0.14}^{+0.13}$ & $0.93_{-0.10}^{+0.10}$ & $514_{-53}^{+54}$ & $1.06_{-0.10}^{+0.10}$ & $582_{-54}^{+53}$ \\
\hline $5634-5688$ & $0.1218_{-0.0010}^{+0.0010}$ & $0.43_{-0.08}^{+0.09}$ & $0.16_{-0.13}^{+0.13}$ & $1.02_{-0.10}^{+0.10}$ & $565_{-53}^{+57}$ & $1.23_{-0.08}^{+0.08}$ & $682_{-43}^{+47}$ \\
\hline
\end{tabular}

these optical data have been uniformly underestimated by $\sim 30 \%$, this gray cloud scenario would only be excluded at $\sim 1 \sigma$ confidence. However, lacking any reason to doubt our inferred $R_{\mathrm{p}} / R_{\star}$ uncertainties, we propose instead that the red data exhibit significant spectral variations that cannot be explained by a gray cloud deck.

\subsection{Forward Model Comparison with Optical-NIR Data}

The results of the previous section imply that the transmission spectrum of WASP-121b exhibits significant wavelengthdependent opacity variations across the $\sim 0.47-1 \mu \mathrm{m}$ wavelength range. To explore this further, we used the ATMO code (Amundsen et al. 2014; Tremblin et al. 2015, 2016; Drummond et al. 2016; Goyal et al. 2018) to generate a small grid of aerosolfree atmosphere models spanning temperature and metallicity, assuming isothermal pressure-temperature $(P T)$ profiles and chemical equilibrium abundances. Specifically, our grid consisted of temperatures ranging from 1000 to $2700 \mathrm{~K}$ in $100 \mathrm{~K}$ increments, each evaluated for metallicities of $0.1 \times, 1 \times, 10 \times$, $20 \times, 30 \times, 40 \times$, and $50 \times$ solar. ATMO solves for the gas-phase and condensed-phase chemical equilibrium mole fractions for a given pressure, temperature, and set of elemental abundances (Drummond et al. 2016). For the results presented here, we consider local condensation, such that the chemistry calculation in each model pressure level is entirely independent of all other pressure levels. We do not account for rainout chemistry, under which condensation deeper within the atmosphere depletes elemental abundances at lower pressure levels (Burrows \& Sharp 1999; Madhusudhan et al. 2011; Mbarek \& Kempton 2016). Rainout could be important in the atmosphere of WASP-121b, but we defer investigation of this effect to future work that includes a more realistic treatment of the $P T$ profile than the isothermal assumption made here. Finally, we applied uniform vertical offsets to $R_{\mathrm{p}} / R_{\star}$ for each model in order to optimize the match to the data. No further tuning of the models was performed.

None of these equilibrium models are able to explain the absorption at wavelengths shortward of $0.47 \mu \mathrm{m}$, nor the G141 bump between wavelengths of $1.15-1.3 \mu \mathrm{m}$. We discuss these latter two components of the transmission spectrum further in Sections 5.3 and 5.4, respectively. For the remaining datanamely, the STIS data spanning the $0.47-1 \mu \mathrm{m}$ wavelength range and the WFC3 data covering the $\mathrm{H}_{2} \mathrm{O}$ band centered at $1.4 \mu \mathrm{m}$ - we find a good match is obtained for the model with a temperature of $1500 \mathrm{~K}$ and metallicity of $20 \times$ solar (Figure 11), which has a reduced $\chi^{2}$ of 1.0 for 69 degrees of freedom. Similarly good matches to the data are obtained for the $1500 \mathrm{~K}$ models with metallicities of $10 \times$ and $30 \times$ solar. These metallicities are broadly consistent with predictions for a $1.18 M_{\mathrm{J}}$ planet such as WASP-121b (Thorngren et al. 2016). 
Table 3

Similar to Table 2, but for the G750L Spectroscopic Light Curve Fits

\begin{tabular}{|c|c|c|c|c|c|}
\hline$\lambda(\AA)$ & $R_{\mathrm{p}} / R_{\star}$ & $u_{1}$ & $u_{2}$ & $\beta$ & $\sigma(\mathrm{ppm})$ \\
\hline $5263-5550$ & $0.1205_{-0.0007}^{+0.0008}$ & $0.36_{-0.08}^{+0.07}$ & $0.30_{-0.12}^{+0.12}$ & $1.02_{-0.07}^{+0.08}$ & $403_{-29}^{+30}$ \\
\hline $5550-5648$ & $0.1220_{-0.0010}^{+0.0010}$ & $0.39_{-0.08}^{+0.08}$ & $0.22_{-0.13}^{+0.14}$ & $1.04_{-0.07}^{+0.08}$ & $609_{-43}^{+45}$ \\
\hline $5648-5745$ & $0.1209_{-0.0011}^{+0.0010}$ & $0.36_{-0.09}^{+0.08}$ & $0.30_{-0.13}^{+0.13}$ & $1.05_{-0.08}^{+0.08}$ & $597_{-45}^{+47}$ \\
\hline $5745-5843$ & $0.1211_{-0.0012}^{+0.0012}$ & $0.31_{-0.09}^{+0.09}$ & $0.31_{-0.13}^{+0.13}$ & $1.03_{-0.10}^{+0.09}$ & $569_{-52}^{+50}$ \\
\hline $5843-5940$ & $0.1243_{-0.0007}^{+0.0008}$ & $0.25_{-0.08}^{+0.08}$ & $0.28_{-0.13}^{+0.12}$ & $1.03_{-0.07}^{+0.08}$ & $563_{-40}^{+43}$ \\
\hline $5940-6038$ & $0.1218_{-0.0008}^{+0.0008}$ & $0.26_{-0.08}^{+0.08}$ & $0.26_{-0.13}^{+0.13}$ & $1.04_{-0.07}^{+0.07}$ & $576_{-39}^{+41}$ \\
\hline $6233-6330$ & $0.1245_{-0.0008}^{+0.0009}$ & $0.30_{-0.08}^{+0.09}$ & $0.20_{-0.13}^{+0.13}$ & $1.05_{-0.08}^{+0.08}$ & $575_{-42}^{+45}$ \\
\hline $6330-6428$ & $0.1202_{-0.0011}^{+0.0011}$ & $0.30_{-0.09}^{+0.09}$ & $0.23_{-0.13}^{+0.14}$ & $1.08_{-0.09}^{+0.08}$ & $583_{-48}^{+45}$ \\
\hline $6428-6526$ & $0.1219_{-0.0008}^{+0.0008}$ & $0.22_{-0.09}^{+0.09}$ & $0.22_{-0.13}^{+0.13}$ & $1.01_{-0.07}^{+0.08}$ & $547_{-40}^{+44}$ \\
\hline $6526-6623$ & $0.1238_{-0.0009}^{+0.0008}$ & $0.21_{-0.09}^{+0.09}$ & $0.18_{-0.13}^{+0.13}$ & $1.13_{-0.07}^{+0.08}$ & $634_{-39}^{+44}$ \\
\hline $6623-6721$ & $0.1225_{-0.0009}^{+0.0008}$ & $0.28_{-0.08}^{+0.08}$ & $0.21_{-0.13}^{+0.13}$ & $0.96_{-0.08}^{+0.09}$ & $532_{-45}^{+48}$ \\
\hline $6721-6818$ & $0.1212_{-0.0012}^{+0.0010}$ & $0.19_{-0.09}^{+0.09}$ & $0.25_{-0.13}^{+0.13}$ & $1.03_{-0.10}^{+0.10}$ & $572_{-57}^{+54}$ \\
\hline 7209-7307 & $0.1225_{-0.0009}^{+0.0008}$ & $0.22_{-0.09}^{+0.09}$ & $0.23_{-0.13}^{+0.13}$ & $1.04_{-0.07}^{+0.08}$ & $627_{-44}^{+47}$ \\
\hline 7307-7404 & $0.1238_{-0.0011}^{+0.0011}$ & $0.18_{-0.09}^{+0.09}$ & $0.22_{-0.13}^{+0.13}$ & $1.05_{-0.09}^{+0.09}$ & $655_{-54}^{+55}$ \\
\hline 7404-7502 & $0.1233_{-0.0008}^{+0.0009}$ & $0.07_{-0.09}^{+0.09}$ & $0.29_{-0.14}^{+0.13}$ & $1.06_{-0.07}^{+0.07}$ & $684_{-46}^{+46}$ \\
\hline $7502-7600$ & $0.1235_{-0.0009}^{+0.0009}$ & $0.16_{-0.09}^{+0.09}$ & $0.16_{-0.13}^{+0.13}$ & $0.96_{-0.08}^{+0.08}$ & $631_{-50}^{+51}$ \\
\hline $7600-7698$ & $0.1240_{-0.0014}^{+0.0013}$ & $0.21_{-0.09}^{+0.09}$ & $0.30_{-0.14}^{+0.14}$ & $1.07_{-0.08}^{+0.08}$ & $722_{-55}^{+55}$ \\
\hline $7698-7795$ & $0.1220_{-0.0012}^{+0.0012}$ & $0.12_{-0.09}^{+0.09}$ & $0.32_{-0.13}^{+0.14}$ & $1.14_{-0.08}^{+0.08}$ & $803_{-54}^{+57}$ \\
\hline 7795-7991 & $0.1203_{-0.0013}^{+0.0014}$ & $0.16_{-0.09}^{+0.10}$ & $0.26_{-0.13}^{+0.13}$ & $1.10_{-0.10}^{+0.09}$ & $595_{-52}^{+50}$ \\
\hline 7991-8186 & $0.1239_{-0.0010}^{+0.0009}$ & $0.16_{-0.09}^{+0.09}$ & $0.27_{-0.13}^{+0.13}$ & $1.01_{-0.07}^{+0.08}$ & $609_{-44}^{+47}$ \\
\hline $8186-8381$ & $0.1219_{-0.0011}^{+0.0011}$ & $0.16_{-0.09}^{+0.09}$ & $0.25_{-0.14}^{+0.13}$ & $1.06_{-0.08}^{+0.08}$ & $699_{-50}^{+51}$ \\
\hline $8381-8840$ & $0.1208_{-0.0007}^{+0.0007}$ & $0.08_{-0.08}^{+0.08}$ & $0.32_{-0.13}^{+0.12}$ & $0.93_{-0.08}^{+0.08}$ & $432_{-35}^{+37}$ \\
\hline 8840-9299 & $0.1216_{-0.0008}^{+0.0008}$ & $0.10_{-0.09}^{+0.08}$ & $0.20_{-0.13}^{+0.13}$ & $1.11_{-0.07}^{+0.08}$ & $548_{-36}^{+40}$ \\
\hline
\end{tabular}

Table 4

Results of Free-chemistry Retrieval Analysis

\begin{tabular}{lc}
\hline \hline Parameter & Value \\
\hline$R_{\text {mbar }}\left(R_{\mathrm{J}}\right)$ & $1.747_{-0.006}^{+0.008}$ \\
$T_{\operatorname{limb}}(\mathrm{K})$ & $1554_{-271}^{+241}$ \\
$\log _{10}\left[\mathrm{H}_{2} \mathrm{O}\right]$ & $-2.2_{-0.3}^{+0.3}$ \\
$\log _{10}[\mathrm{VO}]$ & $-6.6_{-0.3}^{+0.2}$ \\
$\log _{10}[\mathrm{TiO}]^{\mathrm{a}}$ & $<-7.9$ \\
$\log _{10}[\mathrm{Na}]$ & $-2.4_{-0.7}^{+0.4}$ \\
$\log _{10}[\mathrm{FeH}]$ & $-3.7_{-0.4}^{+0.4}$ \\
$\left.\ln _{[} \sigma_{\mathrm{cloud}} / \sigma_{0}\right]$ & $-6.3_{-2.1}^{+2.5}$ \\
\hline
\end{tabular}

Note.

a $3 \sigma$ upper limit.

Aside from collision-induced absorption and gas-phase Rayleigh scattering, the primary opacity sources of these models are $\mathrm{Na}$ and $\mathrm{VO}$ at optical wavelengths and $\mathrm{H}_{2} \mathrm{O}$ at NIR wavelengths. This is illustrated in Figure 13, which shows a breakdown of the opacity sources in the best-matching chemical equilibrium model. Interestingly, opacity due to $\mathrm{TiO}$ is not as significant as that due to $\mathrm{VO}$ in the optical, despite $\mathrm{Ti}$ being approximately an order of magnitude more abundant than V for solar elemental composition (Asplund et al. 2009).
This occurs because for a given pressure, the condensation of $\mathrm{Ti}$ species commences at higher temperatures than for $\mathrm{V}$ species (e.g., Burrows \& Sharp 1999; Woitke et al. 2018). The isothermal temperature of the best-match model (i.e., $1500 \mathrm{~K}$ ) is less than the condensation temperature of both $\mathrm{Ti}_{3} \mathrm{O}_{5}(\mathrm{~s})$ and $\mathrm{V}_{2} \mathrm{O}_{3}(\mathrm{~s})$, meaning that these are the dominant forms of $\mathrm{Ti}$ and $\mathrm{V}$ in the model, respectively. However, since the isothermal temperature is closer to the $\mathrm{VO}(\mathrm{g}) / \mathrm{V}_{2} \mathrm{O}_{3}(\mathrm{~s})$ condensation temperature than the $\mathrm{TiO}(\mathrm{g}) / \mathrm{Ti}_{3} \mathrm{O}_{5}(\mathrm{~s})$ condensation temperature, the abundance of $\mathrm{VO}(\mathrm{g})$ is larger than for $\mathrm{TiO}(\mathrm{g})$.

In contrast, Lodders (2002) found that calcium titanates (e.g., $\mathrm{CaTiO}_{5}$ ) — which are not currently included in ATMOare likely to be the first Ti-bearing condensates to form. Furthermore, arguing from trends in solar system meteorite data and $M / L$ dwarf spectra, Lodders notes that $\mathrm{V}$ will likely condense in solid solution with the calcium titanates, resulting in VO gas-phase depletion commencing at the same temperature as $\mathrm{TiO}$ gas-phase depletion. However, for hot Jupiters, Ti and V condensation may depend on condensation and mixing timescales, both vertical and horizontal, that are very different from those in the protostellar nebula and $M / L$ dwarfs. Such details are complex and beyond the scope of the present study. At this stage, we simply note that VO absorption is favored by these HST data for WASP-121b, with no evidence for significant $\mathrm{TiO}$ absorption, an interpretation 

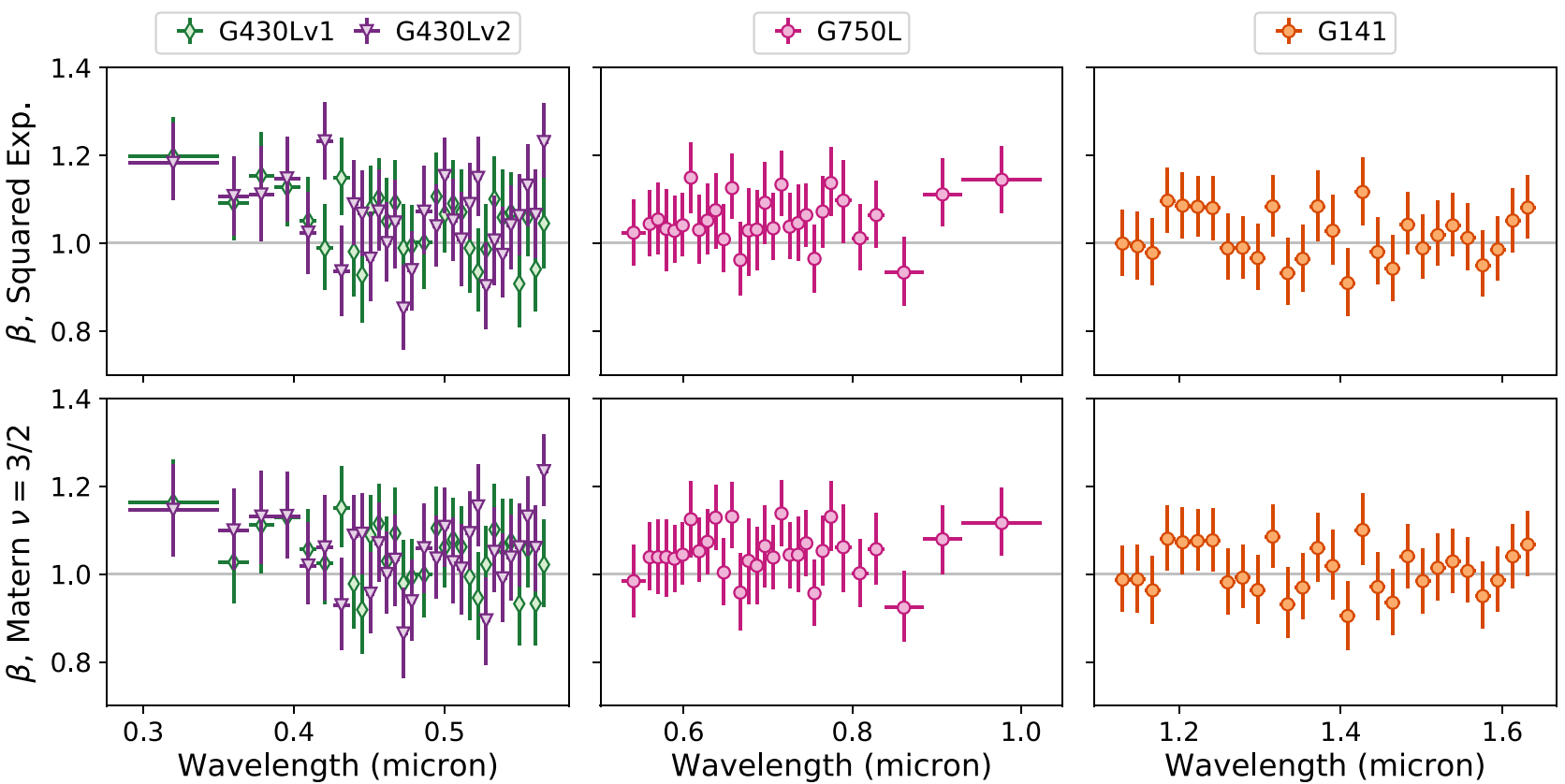

Figure 10. (Top row) Inferred white-noise rescaling parameters $\beta$ for the GP analyses adopting a squared-exponential covariance kernel. (Bottom row) The same, but for the GP analyses adopting a Matérn $\nu=3 / 2$ covariance kernel.

that is corroborated by the free-chemistry retrieval presented in Section 5.4 below.

We also note that the best-matching forward model temperature of $1500 \mathrm{~K}$ is substantially cooler than that of the dayside photosphere, which is inferred to be $\sim 2700 \mathrm{~K}$ from secondary eclipse measurements (Evans et al. 2017). Such a large temperature difference between the dayside photosphere probed during secondary eclipse and the upper atmosphere of the day-night limb probed during primary transit is in fact broadly in line with predictions of 3D general circulation models of ultra-hot Jupiters (e.g., Kataria et al. 2016). Furthermore, the best-match temperature of $1500 \mathrm{~K}$ is likely to be at the lower end of the plausible range, because, as noted above, the forward models we consider here do not include rainout chemistry. Rainout chemistry will likely result in VO condensing at higher temperatures, as the abundance of $\mathrm{VO}$ in the upper atmosphere would be determined by the atmospheric temperature profile at higher pressures where the condensation temperature is also higher. Since the appearance or disappearance of VO spectral bands is primarily what determines the ability of our forward models to match the data (Figure 11), forward models with rainout chemistry would consequently tend to favor higher temperatures. As noted above, we do not consider models with rainout here, as the details will be highly sensitive to the atmospheric $P T$ profile at pressures $>0.1$ bar, which is unconstrained by the current data.

\subsection{Absorption at NUV Wavelengths}

We now consider the steep rise in the transmission spectrum at wavelengths shortward of $\sim 0.47 \mu \mathrm{m}$. As explained in Section 5.1, we consider it unlikely that this feature can be explained by Rayleigh scattering due to gas-phase species such as $\mathrm{H}_{2}$ or high-altitude aerosols. In addition, our chemical equilibrium models presented in Section 5.2 do not predict significant absorption above the $\mathrm{H}_{2}$ continuum at these wavelengths. Nonetheless, we find that the rise of the transmission spectrum at NUV wavelengths is empirically repeatable. It is recovered by our analysis when the spectroscopic light curves for the two G430L visits are fit jointly and also when they are each fit individually (see Appendix B.4).

One candidate absorber is the mercapto radical, SH, comprising a sulfur atom and a hydrogen atom. Indeed, $\mathrm{SH}$ was predicted by Zahnle et al. (2009, hereafter Z09) to be a strong NUV absorber in hot Jupiter atmospheres. Using a 1D photochemical kinetics code, Z09 found that the abundance of SH may peak at pressures around $\sim 1-100$ mbar in typical hot Jupiter atmospheres, with a mixing ratio of $\sim 10 \mathrm{ppm}$ (see Figure 2 of Z09). At these pressures, $\mathrm{H}_{2} \mathrm{~S}$ is the most abundant sulfur-bearing phase under chemical equilibrium (Visscher et al. 2006), while atomic $\mathrm{H}$ and $\mathrm{S}$ are also available due to photodissociation of molecules such as $\mathrm{H}_{2}$ and $\mathrm{H}_{2} \mathrm{O}$. The production of $\mathrm{SH}$ then proceeds through numerous chemical pathways involving $\mathrm{H}_{2} \mathrm{~S}, \mathrm{H}$, and $\mathrm{S}$ (Z09; see also Zahnle et al. 2016).

To explore whether or not SH can explain the observed NUV absorption, we performed a simple fit to the 13 shortestwavelength data points of the transmission spectrum, spanning the $0.3-0.47 \mu \mathrm{m}$ wavelength range (i.e., the blue G430L data subset indicated by the light blue halos in Figures 11 and 12). As in Section 5.1, we followed the methodology outlined in L08. We computed the change in relative planetary radius due to $\mathrm{SH}$ absorption, adopting a planetary surface gravity $g=940 \mathrm{~cm} \mathrm{~s}^{-2}$ and stellar radius $R_{\star}=1.458 R_{\odot}$ (Delrez et al. 2016). We also assumed $\mu=2.22$ atomic mass units (see Section 4) and set $R_{\mathrm{p}} / R_{\star}=0.120$ as the altitude where $\mathrm{H}_{2}$ becomes optically thick at grazing geometry for a wavelength of $\lambda_{0}=350 \mathrm{~nm}$ (Figure 11), corresponding to a planetary radius of $R_{p}=1.702 R_{\mathrm{J}}$. This in turn translates to an atmospheric pressure of $\sim 20 \mathrm{mbar}$, assuming a temperature of $\sim 1500-2000 \mathrm{~K}$ and an $\mathrm{H}_{2}$ scattering cross-section of $\sigma_{0}=$ $3.51 \times 10^{-27} \mathrm{~cm}^{2}$ molecule ${ }^{-1}$ for $\lambda_{0}=350 \mathrm{~nm}$ (see Section 4.1 of L08; also, Sing et al. 2016). Having thus established the pressure scale, we took the temperature-dependent absorption cross-sections for $\mathrm{SH}$ and varied the mixing ratio to optimize 


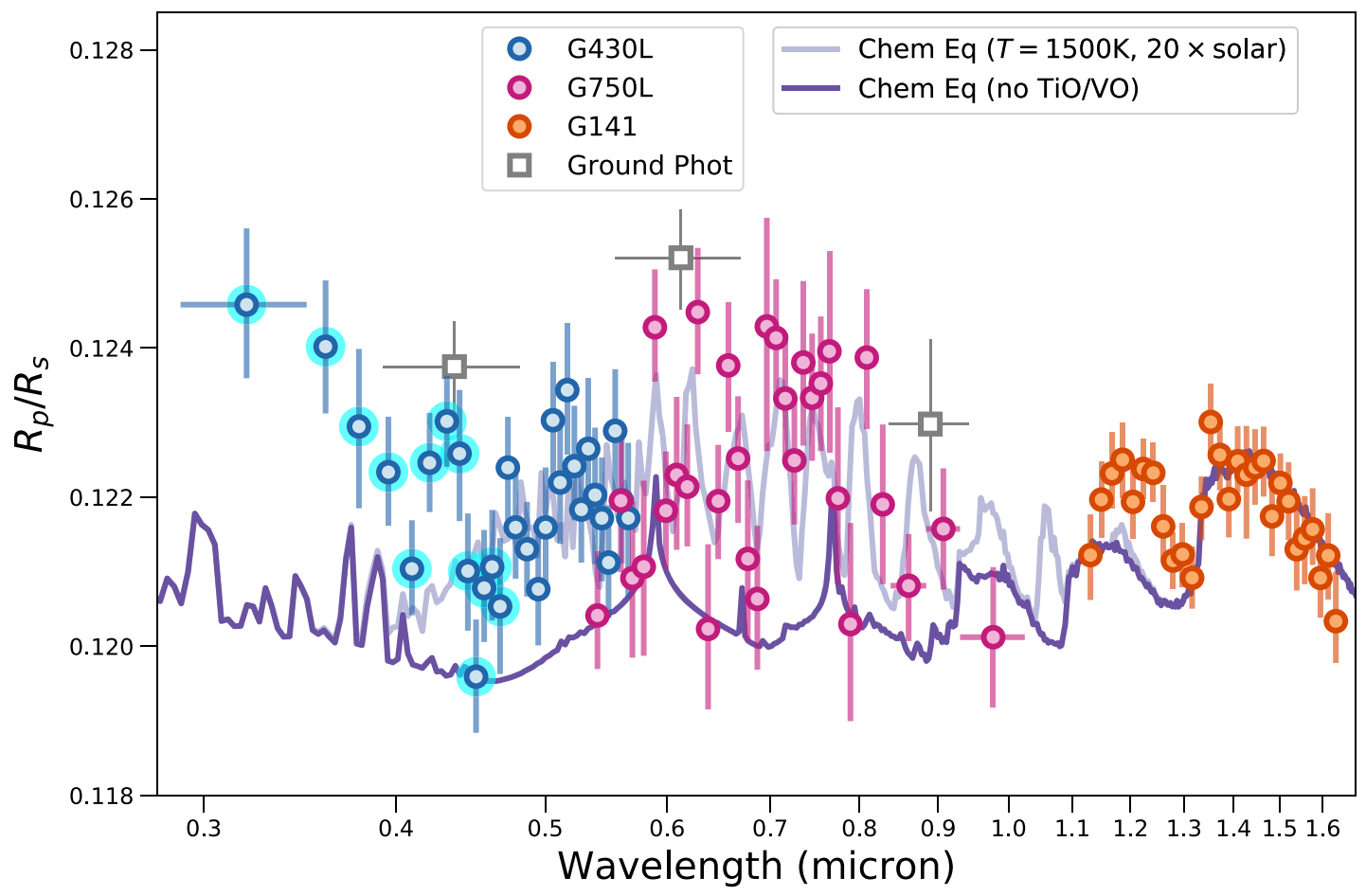

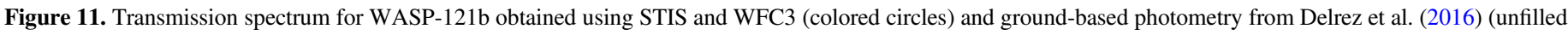

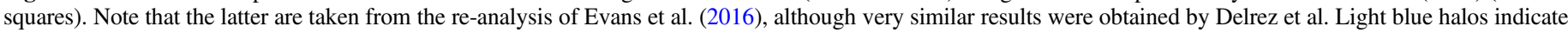

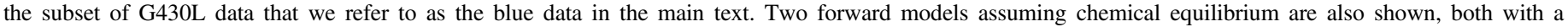

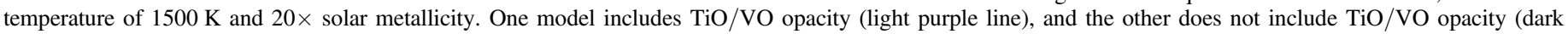
purple line).

the match to the NUV transmission spectrum using Equation (1) of L08. For the SH cross-sections, we combined those derived by Z09 with those recently published by the ExoMol project (Yurchenko et al. 2018). Specifically, the Z09 crosssections were generated from transitions of the lowest five vibrational levels of the ground electronic state $X^{2} \Pi$ to the lowest three vibrational levels of the upper electronic state $\mathrm{A}^{2} \Sigma^{+}$(without predissociation), and exhibit a strong NUV signature. These transitions are not considered in the ExoMol cross-sections, which only account for rotational-vibrational transitions. Both the Z09 and ExoMol cross-sections are shown in Figure 14.

The results of this process are shown in Figure 12. We obtain respectable matches to the data with mixing ratios of $\sim 100 \mathrm{ppm}$ and $\sim 20 \mathrm{ppm}$, respectively, for the $T=1500 \mathrm{~K}$ and $T=2000 \mathrm{~K}$ absorption cross-sections of Z09. For comparison, Figure 15 shows predicted abundances from the photochemical kinetics code of Z09 for a planet similar to WASP-121b with $20 \times$ solar metallicity and vertical mixing parameter $K_{z z}=10^{9} \mathrm{~cm}^{2} \mathrm{~s}^{-1}$. We find that abundances of $\sim 20-100 \mathrm{ppm}$ are plausible for SH across the bar to mbar pressure range probed by the transmission spectrum, lending some credibility to the hypothesis that it could be the mystery NUV absorber. However, as stressed by Z09, the SH crosssections remain subject to considerable uncertainty, due to the paucity of available experimental data. This, combined with the low spectral resolution of the G430L data, prevents us from conclusively confirming or ruling out $\mathrm{SH}$ at the present time. Other sulfur-bearing compounds that are likely to be abundant, such as SiS, have strong features at NUV wavelengths but remain poorly modeled. Lothringer et al. (2018) have also flagged gas-phase Fe as an important NUV absorber in ultrahot Jupiter atmospheres, although we find it is unable to account for the measured signal in the present data set - at least under assumptions of equilibrium chemistry for pressures $>10^{-5}$ bar-as it was included in the ATMO forward models described in Section 5.2 (see Figure 13).

Regardless of the identity of the putative NUV absorber, it likely provides significant heating of the upper atmosphere. For instance, across the $0.3-0.47 \mu \mathrm{m}$ wavelength range, the mean SH absorption cross-section varies from $\sim 10^{-16}$ to $10^{-22} \mathrm{~cm}^{2}$ molecule ${ }^{-1}$ (Figure 14). Assuming a mixing ratio of $\sim 10$ ppm, in line with our above estimates, this implies a mean atmospheric opacity (i.e., absorption cross-section $\times$ mixing ratio) of $\sim 10^{-24} \mathrm{~cm}^{2}$ molecule ${ }^{-1}$ at the pressures probed in transmission. We find incorporating such an absorber into the 1D radiative-convective atmosphere model of Marley and collaborators (e.g., Marley \& McKay 1999; Marley et al. 2002, 2012; Fortney et al. 2008; Saumon \& Marley 2008) would likely heat the atmosphere of WASP-121b by $\sim 500 \mathrm{~K}$ at mbar pressures. Such heating could, for example, help maintain optical absorbers such as $\mathrm{VO}$ and $\mathrm{TiO}$ in the gas phase, which in turn would provide further heating of the upper atmosphere. Properly accounting for effects such as these may be important for accurate modeling of planetary circulation and energy budgets.

Absorption of incident UV flux exceeding that predicted by models has also been observed in solar system atmospheres. Two well-known examples are Venus and Jupiter. On Jupiter, a broad reflectivity dip near $0.3 \mu \mathrm{m}$ has been attributed to a highaltitude dust or haze (Axel 1972; Owen \& Sagan 1972). The composition of this chromophore is still not known and is 

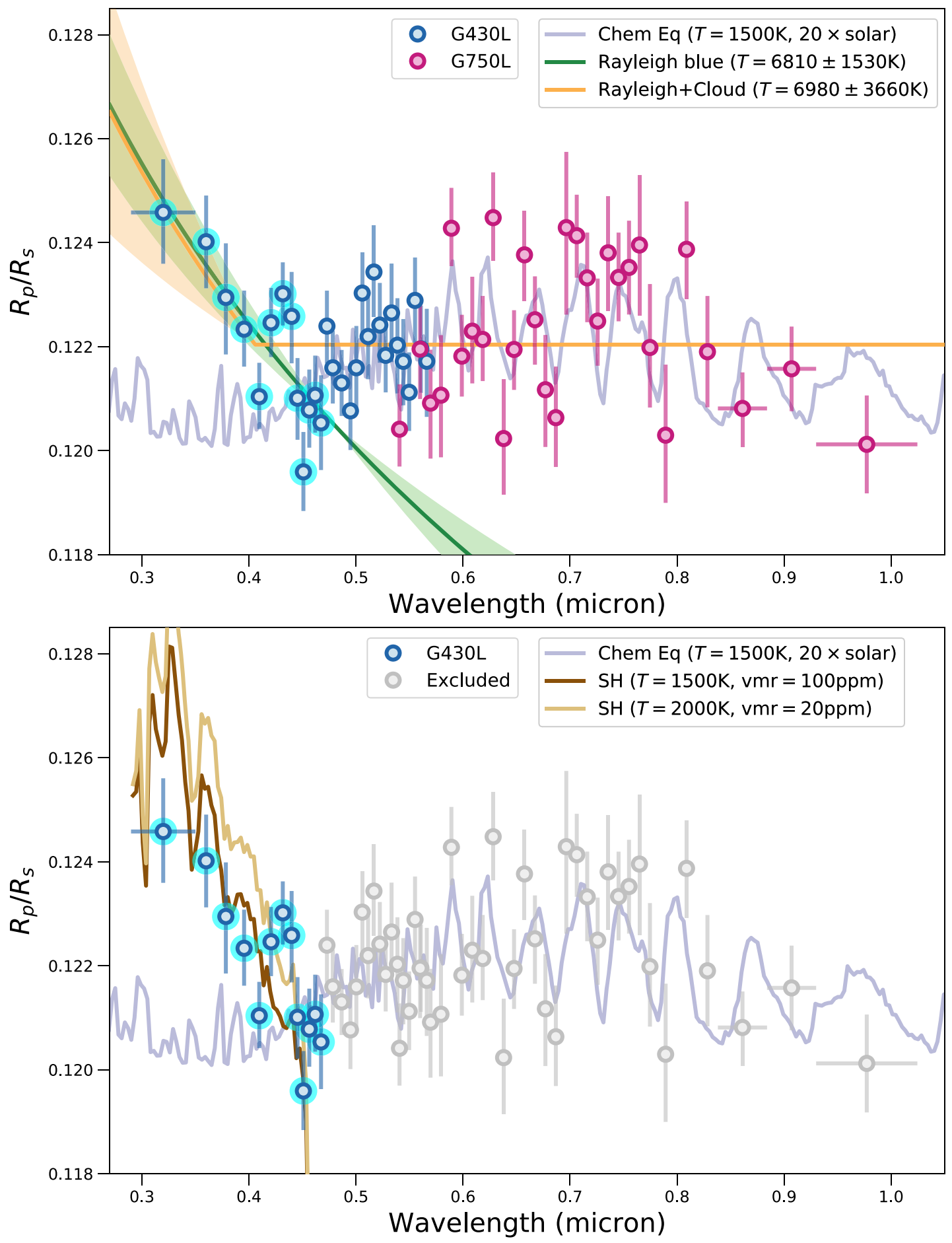

Figure 12. Similar to Figure 11, but showing only the STIS data. (Top panel) Rayleigh scattering fit to the NUV data only (green line) and a hybrid Rayleigh+cloud model fit to the complete STIS data set (yellow line). Although Rayleigh scattering gives a good fit to the NUV data, it requires invoking an unphysically high temperature. The Rayleigh+cloud model is ruled out at $3.7 \sigma$ confidence, due to the opacity variations measured across optical wavelengths. (Bottom panel) Models illustrating the expected opacity contribution due to SH for temperatures of $1500 \mathrm{~K}$ and $2000 \mathrm{~K}$ with volume mixing ratios $100 \mathrm{ppm}$ and $20 \mathrm{ppm}$, respectively (brown lines).

generally attributed to some disequilibrium combination of $\mathrm{S}, \mathrm{N}, \mathrm{C}$, and $\mathrm{P}$ species (for a fuller discussion, see West et al. 2004). Likewise on Venus, dark markings in the atmosphere at UV wavelengths remain poorly understood, well over four decades after their discovery (e.g., Esposito et al. 1997). These features have also been attributed to some disequilibriumperhaps S-bearing-absorber (but see Pollack et al. 1980).

\subsection{Retrieval Analysis of Optical-NIR Data}

In addition to comparing the data with predictions of forward models that assume chemical equilibrium (Section 5.2), we performed a free-chemistry retrieval analysis. For these calculations, we treat the abundances of the radiatively active chemical species as free parameters in the model, rather than 

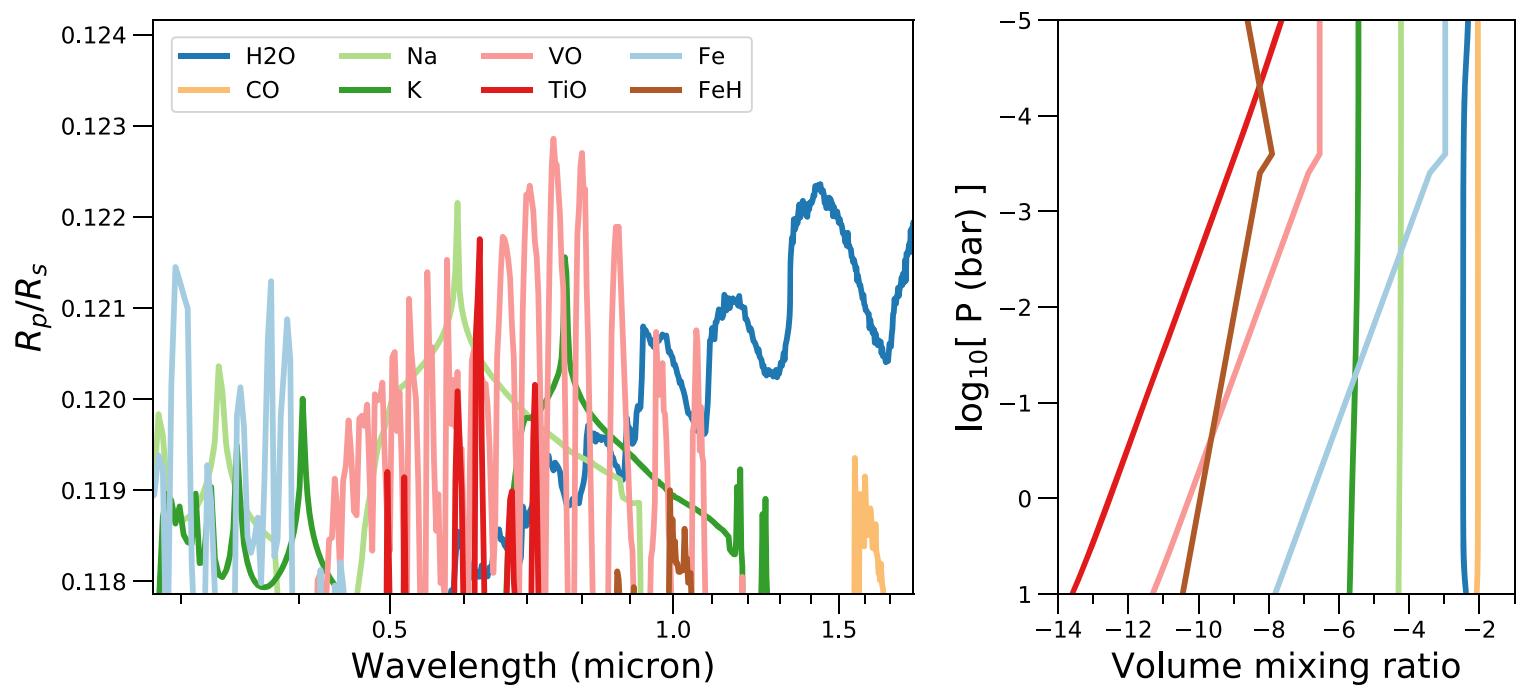

Figure 13. (Left panel) Individual contributions to the transmission spectrum due to the major radiatively active species in the best-match forward model shown in Figure 11, i.e., chemical equilibrium for $T=1500 \mathrm{~K}$ and $20 \times$ solar metallicity. Note that continuum opacity due to gas-phase species such as $\mathrm{H}_{2}$ and $\mathrm{He}$ is not shown. (Right panel) Corresponding pressure-dependent abundances.

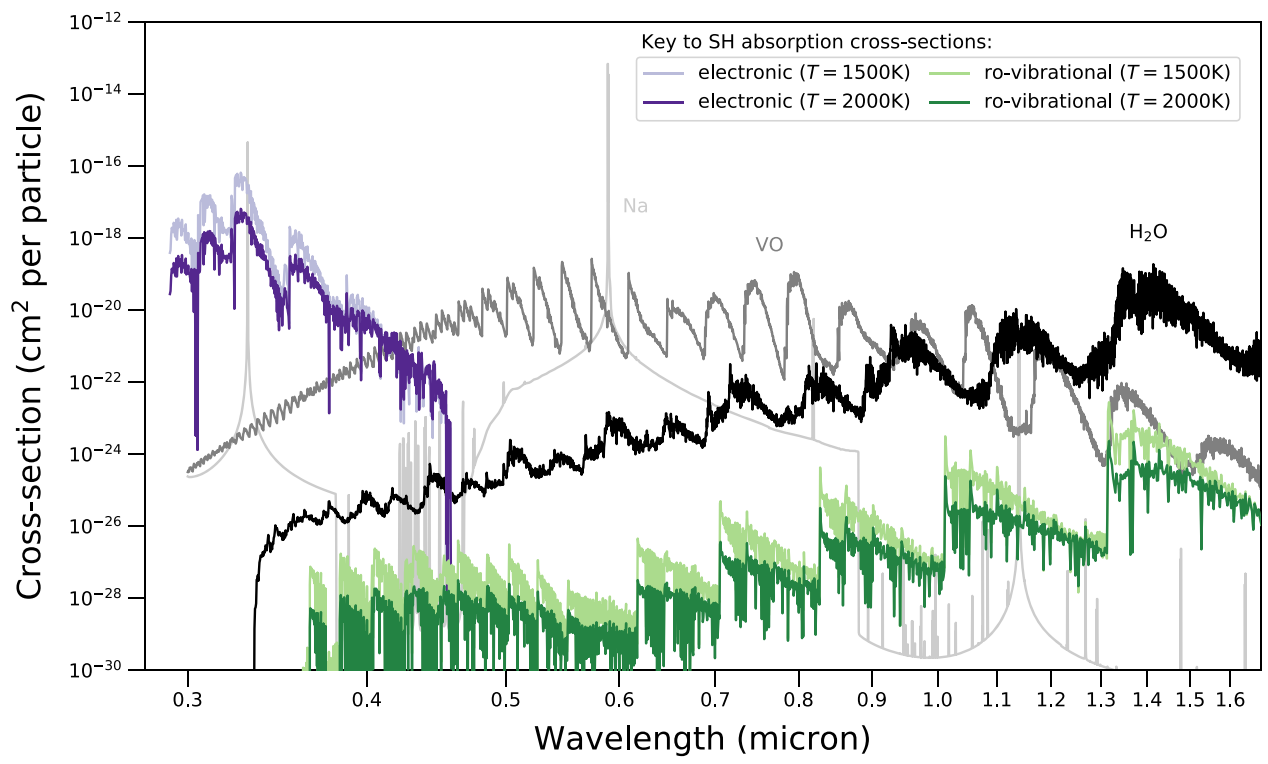

Figure 14. Absorption cross-sections for SH. Electronic transitions are from Zahnle et al. (2009) and rotational-vibrational transitions are from ExoMol (Yurchenko et al. 2018). Cross-sections for $\mathrm{H}_{2} \mathrm{O}$, VO, and $\mathrm{Na}$ are also shown, weighted by the relative abundances implied by the model shown in Figure 13 .

solving for the chemical equilibrium abundances at a given temperature. As for the forward models, this was done using ATMO, which can compute transmission spectra for any given atmospheric composition and $P T$ profile. ATMO has previously been used for retrieval analyses of transmission spectra (Wakeford et al. 2017, 2018) and thermal emission spectra (Evans et al. 2017).

Since ATMO does not currently include any opacity sources that can explain the steep rise observed at NUV wavelengths (Figure 11), we restricted the retrieval to optical-NIR wavelengths longward of $0.47 \mu \mathrm{m}$. We assumed an isothermal $P T$ profile and allowed the limb-averaged temperature $\left(T_{\text {limb }}\right)$ to vary as a free parameter, as well as the reference planet radius corresponding to the 1 mbar pressure level $\left(R_{\text {mbar }}\right)$, effectively providing a floating offset between the model and data. The abundances of $\mathrm{H}_{2} \mathrm{O}$, TiO, $\mathrm{VO}, \mathrm{Na}$, and $\mathrm{FeH}$ were allowed to vary relative to a background atmosphere composition dominated by $\mathrm{H}_{2}$ and $\mathrm{He}$, assuming uniform mixing ratios with pressure. Other gas-phase absorbers such as $\mathrm{K}$ and $\mathrm{CO}$ were fixed to equilibrium abundances for the final analysis, as these were found to be unconstrained by the current data. Opacity due to aerosol Rayleigh scattering and optically thick gray cloud was treated using the approach of Sing et al. (2016). Fitting was performed using differential evolution MCMC (Eastman et al. 2013), as described in our previous works (Evans et al. 2017; Wakeford et al. 2017, 2018).

The inferred distributions for each model parameter are summarized in Table 4 and shown in Figure 16. We find no evidence for opacity contribution due to aerosols, irrespective of whether they are treated as an enhanced Rayleigh scattering component or an optically thick gray cloud. For this reason, we only present the results for the case 


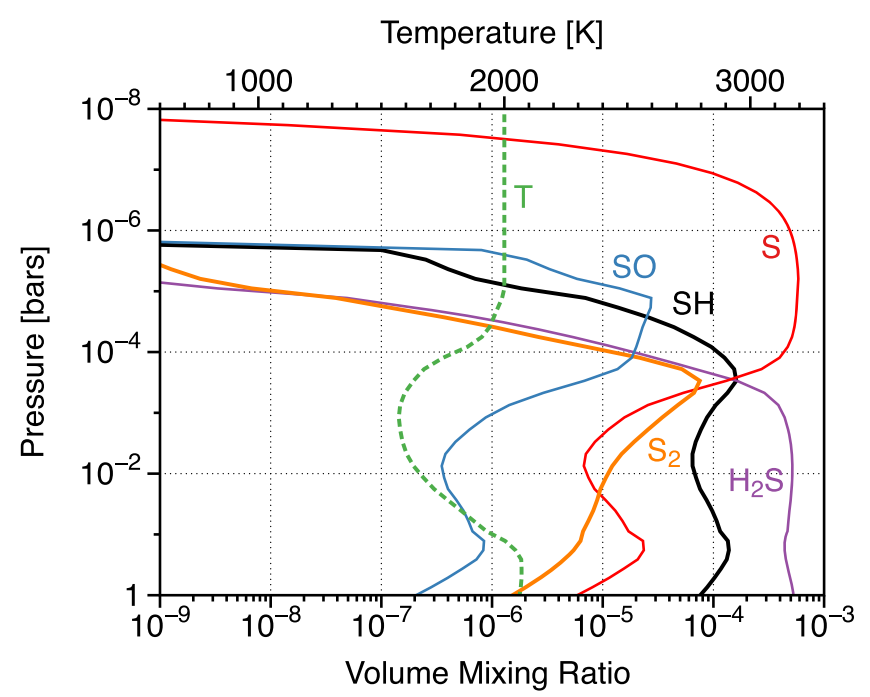

Figure 15. Abundance predictions for important sulfur species assuming $20 \times$ solar metallicity and $K_{z z}=10^{9} \mathrm{~cm}^{2} \mathrm{~s}^{-1}$. The dashed green line indicates the adopted $P T$ profile, based on the limb average of a 3D GCM for WASP-121b (T. Kataria et al. 2018, in preparation). Calculations were performed using the photochemical kinetics code of Zahnle et al. (2009), assuming a planet with a hydrogen-dominated atmosphere orbiting an F6V host star at the same distance as WASP-121b.

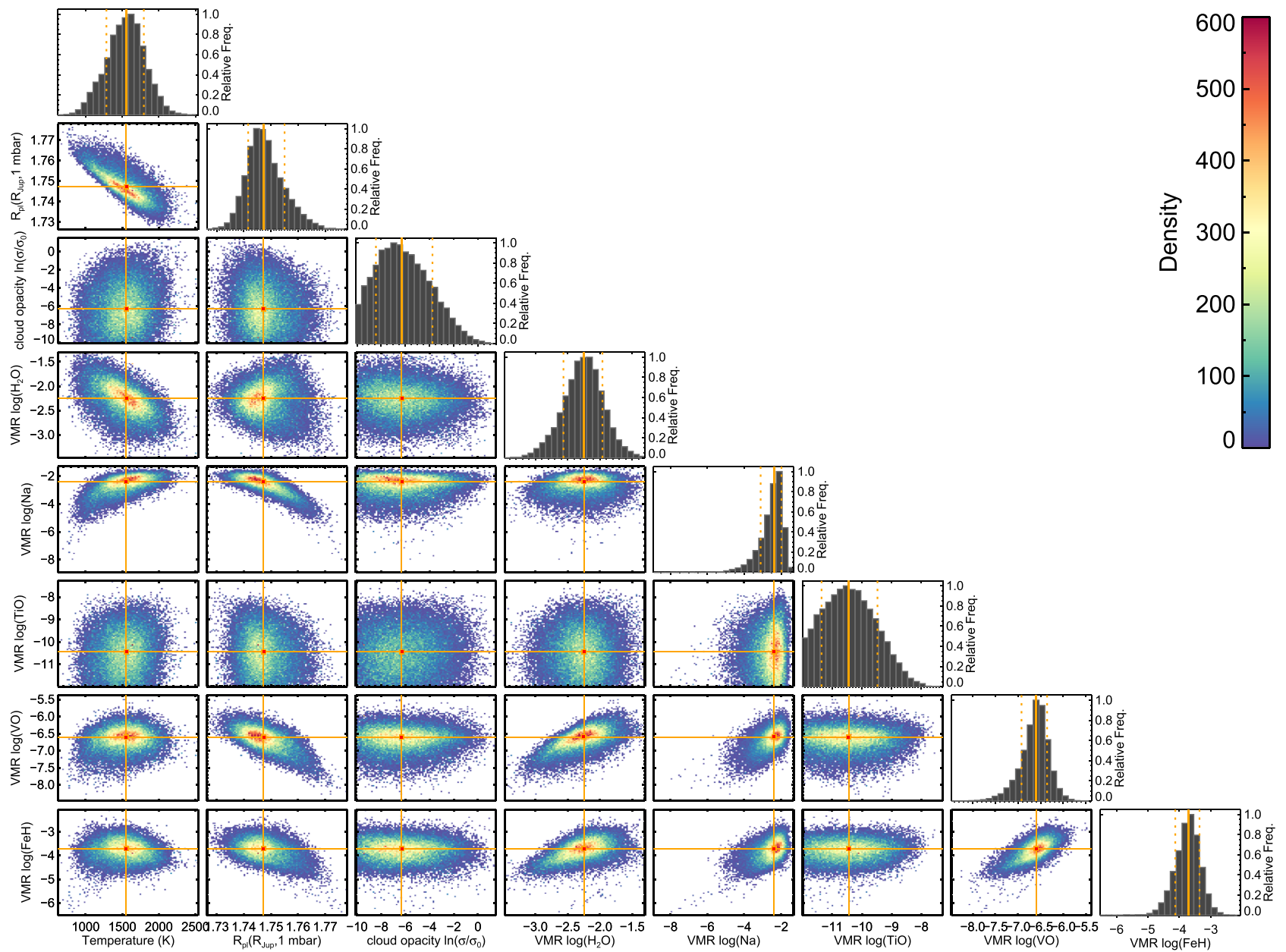

Figure 16. Results of the free-chemistry retrieval analysis. (Off-diagonal panels) Heat maps showing the density of samples drawn from the MCMC analysis for different pairs of parameters. (Diagonal panels) Marginalized density distributions for individual parameters. The solid orange lines indicate parameter median values, and the dashed orange lines indicate ranges spanning $68 \%$ of the samples.

including a gray cloud layer, as the specific treatment of aerosols has negligible impact on the values inferred for the other model parameters.
We obtain a limb-averaged temperature of $T_{\text {limb }}=$ $1554_{-271}^{+241} \mathrm{~K}$, in agreement with the best-matching chemical equilibrium model presented in Section 5.2. The inferred 


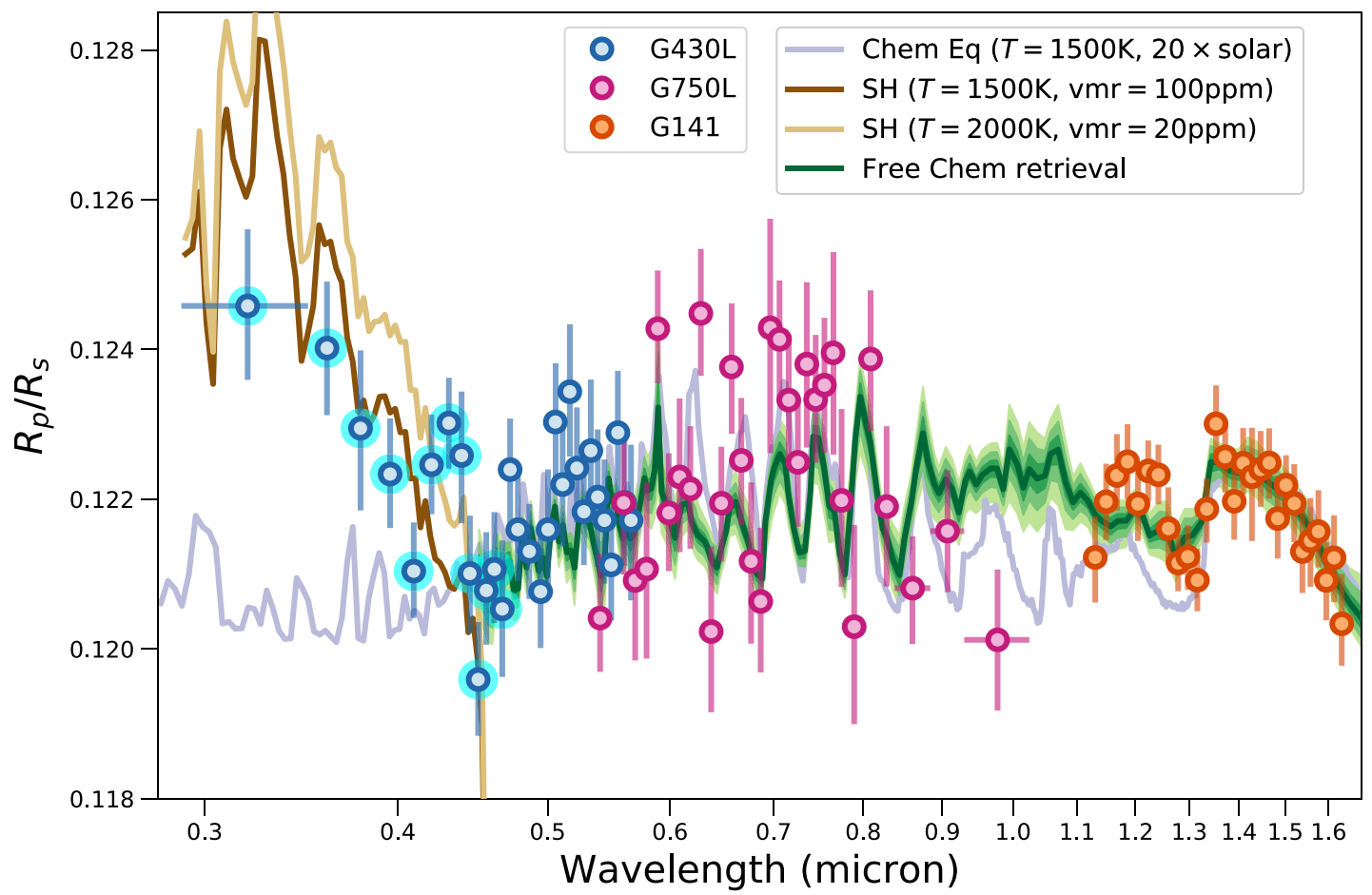

Figure 17. Similar to Figure 11, but showing the distribution of model spectra inferred by the retrieval analysis as well as a hypothetical signal due to SH. The dark green line shows the sample mean at each wavelength, and the shaded green areas progressively encompass $68 \%, 95.5 \%$, and $99.7 \%$ of samples about the mean. A significant departure from the chemical equilibrium model occurs between wavelengths of $\sim 0.9-1.3 \mu \mathrm{m}$. This is due to the retrieval inferring a high FeH abundance to explain the bump in the transmission spectrum measured over the short-wavelength half of the G141 bandpass.

abundances for $\mathrm{H}_{2} \mathrm{O}\left(-2.2_{-0.3}^{+0.3} \mathrm{dex}\right)$, $\mathrm{VO}\left(-6.6_{-0.3}^{+0.2} \mathrm{dex}\right)$, and $\mathrm{TiO}\left(-10.4_{-0.9}^{+1.0} \mathrm{dex}\right)$ are also in good agreement with those predicted by the best-matching equilibrium model (Figure 13). The inferred abundance for $\mathrm{Na}\left(-2.4_{-0.7}^{+0.4}\right.$ dex; $2 \sigma$ lower limit of $-4.22 \mathrm{dex})$ is somewhat higher than the $20 \times$ solar value of -4.24 dex (Figure 13). One possibility is that the core of the $\mathrm{Na}$ line is probing the planetary thermosphere, where temperatures are higher and the pressure scale height is larger. This would produce a strong Na feature that the retrieval may misinterpret as indicating a high abundance. For instance, Huitson et al. (2012) detected a strong Na line in the STIS transmission spectrum for HD 189733b, which high-resolution spectroscopy showed is caused by a thermosphere (Wyttenbach et al. 2015).

In addition, the inferred abundance for $\mathrm{FeH}\left(-3.7_{-0.4}^{+0.4}\right.$ dex) is $\sim 5$ orders of magnitude greater than expected for $20 \times$ solar metallicity (Figure 13). Such a high FeH abundance-which we consider implausible-is driven by the bump in the measured transmission spectrum across the $1.15-1.3 \mu \mathrm{m}$ wavelength range, where $\mathrm{FeH}$ has a significant absorption signature (e.g., see Figure 7 of Sharp \& Burrows 2007). This can be seen clearly in Figure 17, which shows the distribution of spectra implied by the retrieval analysis, compared with the best-matching chemical equilibrium model. The inability of our model to simultaneously explain the $1.15-1.3 \mu \mathrm{m}$ bump and the rest of the data results in a moderately poor overall fit, with a reduced $\chi^{2}$ of 1.5 for 67 degrees of freedom.

The $1.15-1.3 \mu \mathrm{m}$ bump in the transmission spectrum remains puzzling. It has been recovered by multiple independent analyses of the data performed within our own group, as well as those published by others (e.g., Tsiaras et al. 2018). We note that it coincides with a possible spectral feature identified in the dayside thermal spectrum, which was tentatively attributed to VO emission (Evans et al. 2017). However, it is difficult to reconcile VO with the feature seen in the transmission spectrum, as it would require increasing the abundance to a level that would be incompatible with the data at optical wavelengths, where VO has a higher opacity. On the other hand, although the host star is photometrically quiet and care has been taken to precisely measure the absolute transit depths for each bandpass (G430L, G750L, G141), it is conceivable that small offsets in $R_{\mathrm{p}} / R_{\star}$ remain, which, if accounted for, could allow VO to simultaneously explain the transmission spectrum at optical wavelengths along with the $1.15-1.3 \mu \mathrm{m}$ feature. For multi-epoch observations that do not overlap in wavelength such as those considered here, it is impossible to rule out such a scenario with absolute confidence. Upcoming G141 observations should allow a determination of whether or not the $1.15-1.3 \mu \mathrm{m}$ bump is repeatable. It is also worth noting that a strong thermal gradient over the pressures probed in transmission-which has not been considered in the present study-could potentially affect the shape of the transmission spectrum by altering the pressure-dependent scale height $H$ and chemical abundances.

In summary, the retrieval analysis reveals no evidence for aerosols in the optical-NIR transmission spectrum of WASP121b. The inferred limb-averaged temperature and gas-phase abundances are overall in good agreement with the bestmatching forward models of Section 5.2, which assume chemical equilibrium and $10-30 \times$ solar metallicity. The primary exception is the inferred $\mathrm{FeH}$ abundance, which as described above is far higher than expected for chemical equilibrium and $10-30 \times$ solar metallicity. We thus conclude that it is unlikely FeH opacity is the true cause of the spectral bump at wavelengths $1.15-1.3 \mu \mathrm{m}$, the provenance of which remains uncertain. 


\section{Conclusion}

We have presented an STIS transmission spectrum for WASP-121b, spanning the $0.3-1 \mu \mathrm{m}$ wavelength range, adding to the $1.15-1.65 \mu \mathrm{m}$ wavelength coverage of published WFC3 data. The new optical data show an increase in atmospheric opacity for wavelengths shortward of $\sim 0.47 \mu \mathrm{m}$, with a slope that is too steep to be explained by Rayleigh scattering. Instead, assuming the NUV rise is a bona fide signature of the planetary atmosphere, it must be caused by one or more absorbers. We propose $\mathrm{SH}$ as a possible candidate, with a mixing ratio of approximately $\sim 20-100 \mathrm{ppm}$. Although the identity of the NUV absorber remains uncertain, it should cause substantial heating of the upper atmosphere and therefore could be an important component missing from existing models of highly irradiated atmospheres. At longer wavelengths between 0.47 and $1 \mu \mathrm{m}$, we measure significant opacity variations that can be well explained by VO absorption. Analyzing the STIS and WFC3 data with both free-chemistry retrievals and comparisons to chemical equilibrium forward models, we estimate abundances of $\mathrm{H}_{2} \mathrm{O}$ and $\mathrm{VO}$ to be approximately $\sim 10-30 \times$ solar. We find no significant evidence for $\mathrm{TiO}$, suggesting it may have condensed from the gas phase. Our chemical equilibrium forward models are unable to simultaneously reproduce the optical data and the WFC3 bump spanning the $1.15-1.35 \mu \mathrm{m}$ wavelength range. Free-chemistry retrievals are able to do so, but only by invoking an unrealistically high $\mathrm{FeH}$ abundance.

Overall, the evidence uncovered here for significant NUV and optical absorption implies a substantial fraction of incident stellar radiation is likely deposited at low pressures in the atmosphere of WASP-121b. Heating via this mechanism could be responsible for the thermal inversion detected on the dayside hemisphere. The broad coherence of this picture is tantalizing, but many unknowns remain. Although we consider the evidence for $\mathrm{VO}$ in the existing transmission spectrum to be reasonably strong, further observations are required to confirm or rule it out at high confidence. Similarly, additional observations, along with a more extensive exploration of candidates other than $\mathrm{SH}$, are required to identify the NUV absorber. The possible explanation provided by $\mathrm{SH}$, however, flags the potential importance of non-equilibrium sulfur chemistry in highly irradiated atmospheres, which until now has received relatively little attention.

The authors are grateful to the anonymous referee for constructive feedback that improved the quality of this manuscript. We thank Richard Freedman for providing the SH absorption cross-sections. Support for program GO-14767 was provided by NASA through a grant from the Space Telescope Science Institute, which is operated by the Association of Universities for Research in Astronomy, Inc., under
NASA contract NAS 5-26555. T.M.E., D.K.S., N.N., and B.D. acknowledge funding from the European Research Council under the European Union's Seventh Framework Programme (FP7/2007-2013)/ERC grant agreement No. 336792. J.M.G. acknowledges funding from Leverhulme Trust Research Project Grant and University of Exeter PhD Studentship. G.W.H. and M.H.W. acknowledge support from Tennessee State University and the State of Tennessee through its Centers of Excellence program. J.S.F. acknowledges funding by the Spanish MINECO grant AYA2016-79425-C3-2-P. J.K.B. is supported by a Royal Astronomical Society Research Fellowship. L.B.-J. and P.L. acknowledge support from CNES (France) under project PACES. This work has been carried out in the frame of the National Centre for Competence in Research PlanetS supported by the Swiss National Science Foundation (SNSF). V.B. and D.E. have received funding from the European Research Council (ERC) under the European Union's Horizon 2020 research and innovation programme (project FOUR ACES; grant agreement No. 724427).

Facility: HST (STIS and WFC3).

\section{Appendix A Raw Spectroscopic Light Curves}

HST light curves are strongly affected by instrumental systematics that must be accounted for as part of the light curve fitting process. For this reason, we present the raw spectroscopic light curves for the G430Lv1, G430Lv2, and G750L data sets in Figures 18-20, respectively. These figures also show the residuals after dividing the raw spectroscopic light curves by the corresponding white light curve best-fit transit signal, making it easier to inspect the systematics. Red lines indicate the best-fit systematics of the corresponding white light curve in order to highlight the wavelength-dependent nature of the systematics, which must be modeled individually for each spectroscopic channel.

As described in Section 4, however, we do apply a commonmode correction before fitting the spectroscopic light curves. The common-mode corrections are constructed for each data set from the residuals of the corresponding white light curve with the best-fit transit signal removed. Figures 21-23 show the spectroscopic light curves after applying common-mode corrections for the G430Lv1, G430Lv2, and G750L data sets, respectively. Red lines indicate the best-fit GP model for each spectroscopic channel, which includes both the systematics and transit signals. Histograms of residuals are also shown for each spectroscopic channel. These were generated by taking 1000 random draws from the best-fit GP model, subtracting each of these from the data, then binning the resulting residuals. 

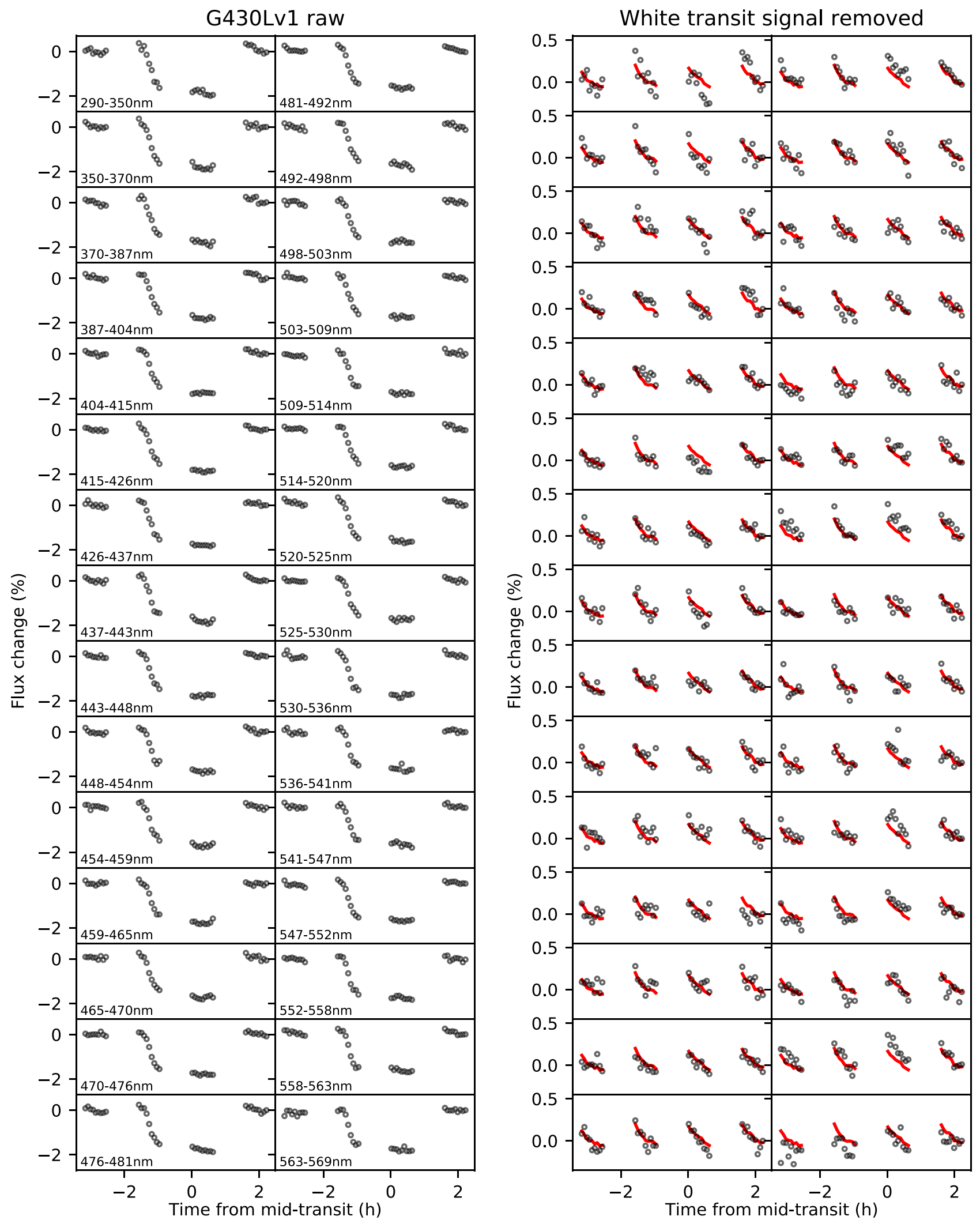

Figure 18. (Left two columns) Raw light curves for each spectroscopic channel of the G430Lv1 data set. (Right two columns) Black circles show residuals after subtracting the best-fit white light curve transit signal from each of the raw spectroscopic light curves, to highlight the systematics component. Red lines show the bestfit white light curve systematics model, to emphasize variation in systematics across spectroscopic channels. 

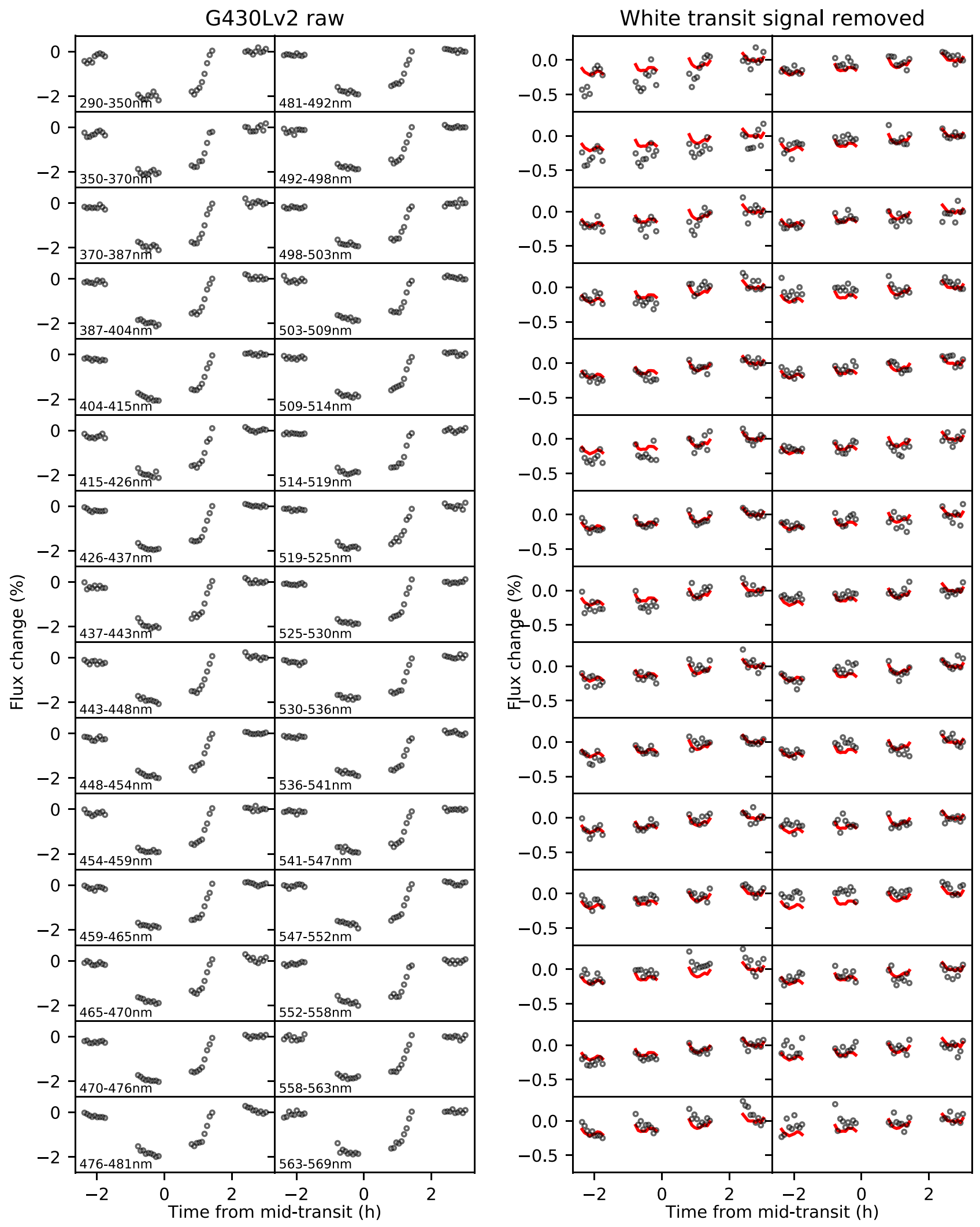

Figure 19. The same as Figure 18, but for the G430Lv2 data set. 

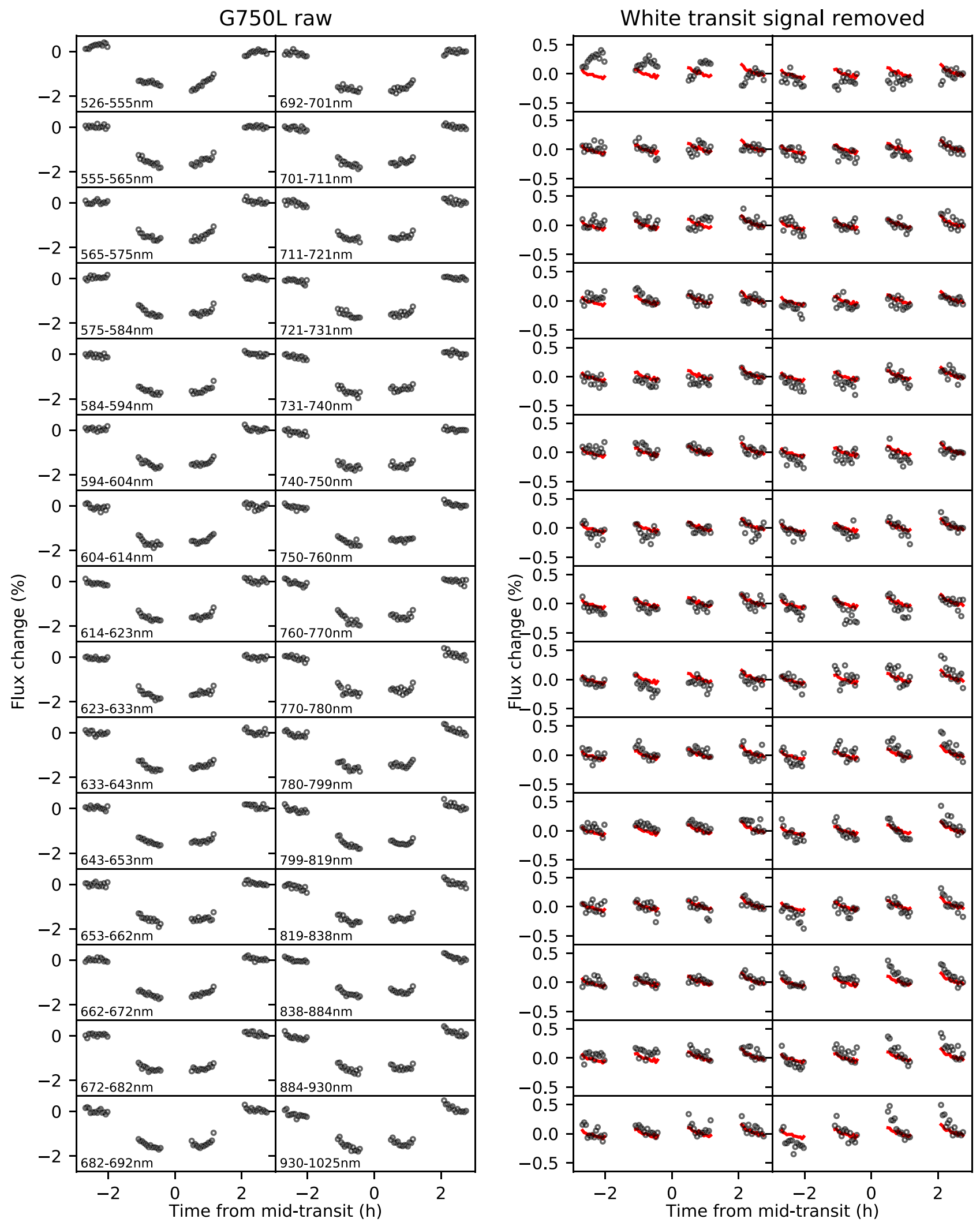

Figure 20. The same as Figure 18, but for the G750L data set. 
G430Lv1 common-mode-corrected

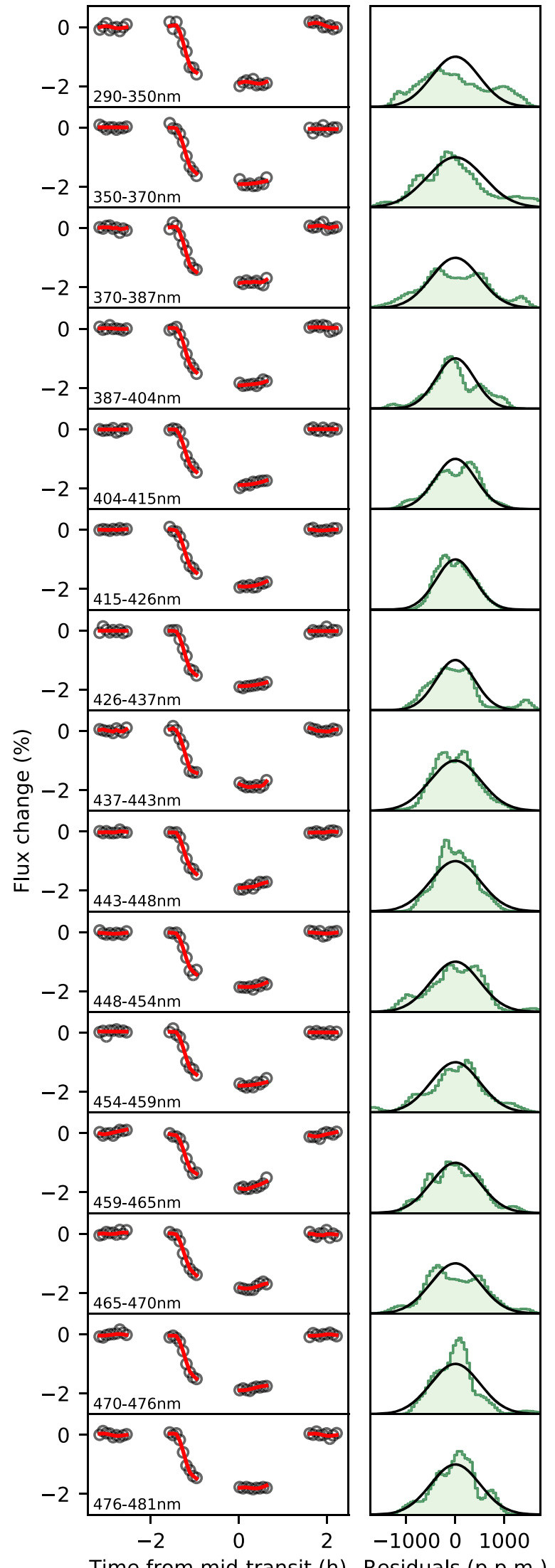

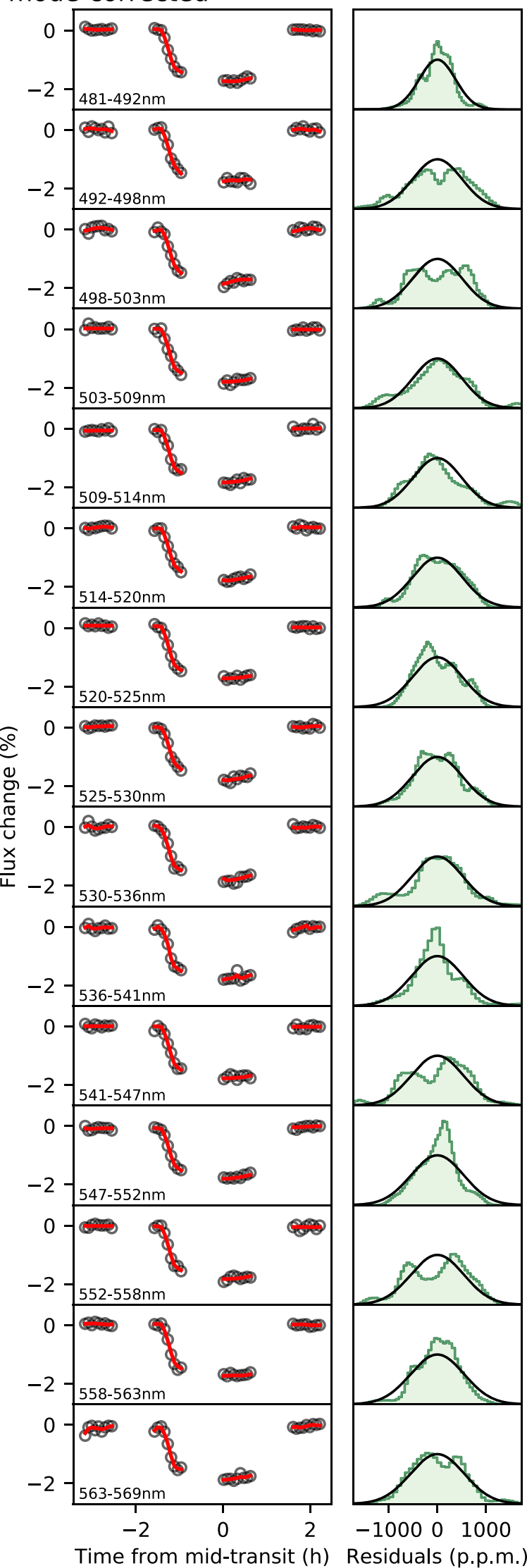

Figure 21. (First and third columns) Black circles show spectroscopic light curves for the G430Lv1 data set after applying a common-mode correction. Red lines show best-fit GP models that simultaneously account for the transit signal and systematics. (Second and fourth columns) Histograms of residuals between the data and bestfit GP model for each spectroscopic light curve, generated the same way as those shown in Figure 4. Black lines show normalized normal distributions with standard deviation equal to photon noise. 
G430Lv2 common-mode-corrected

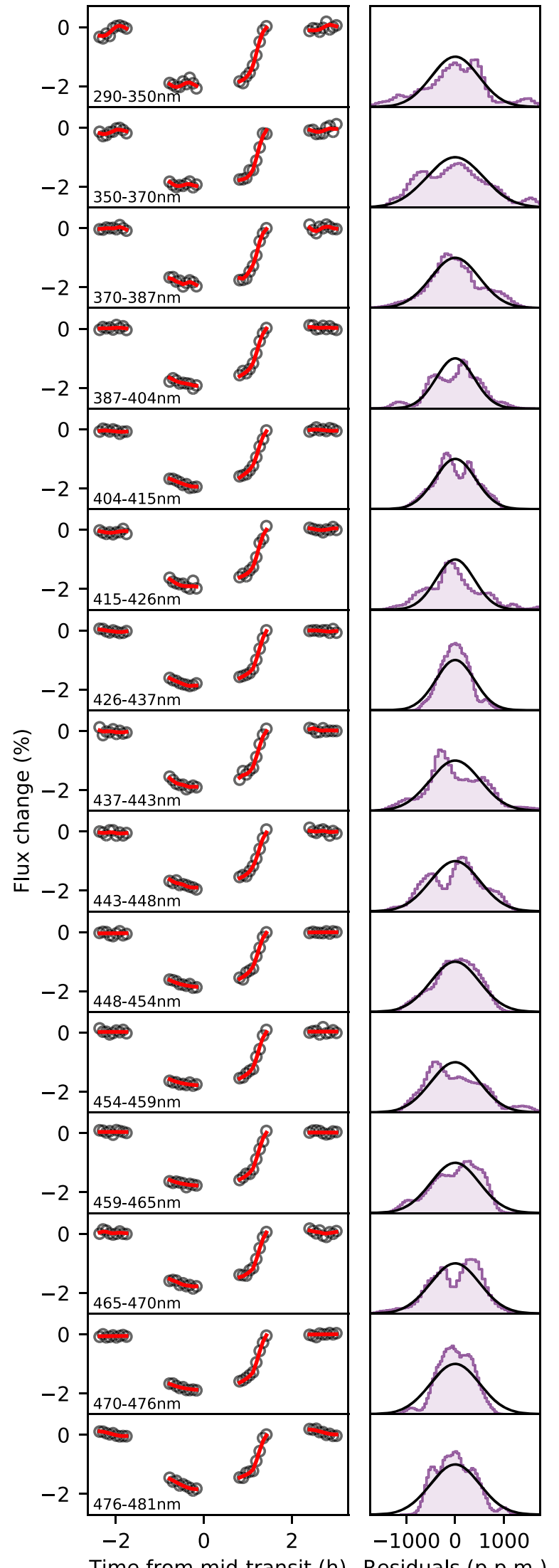

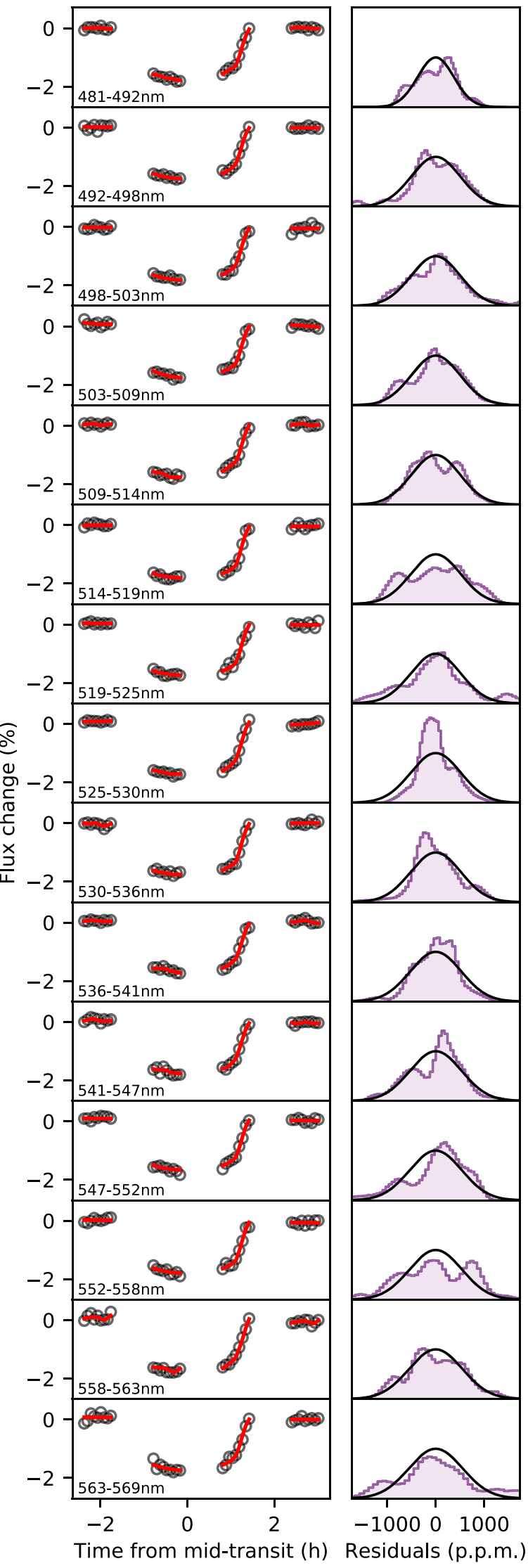

Figure 22. The same as Figure 21, but for the G430Lv2 data set. 
G750L common-mode-corrected
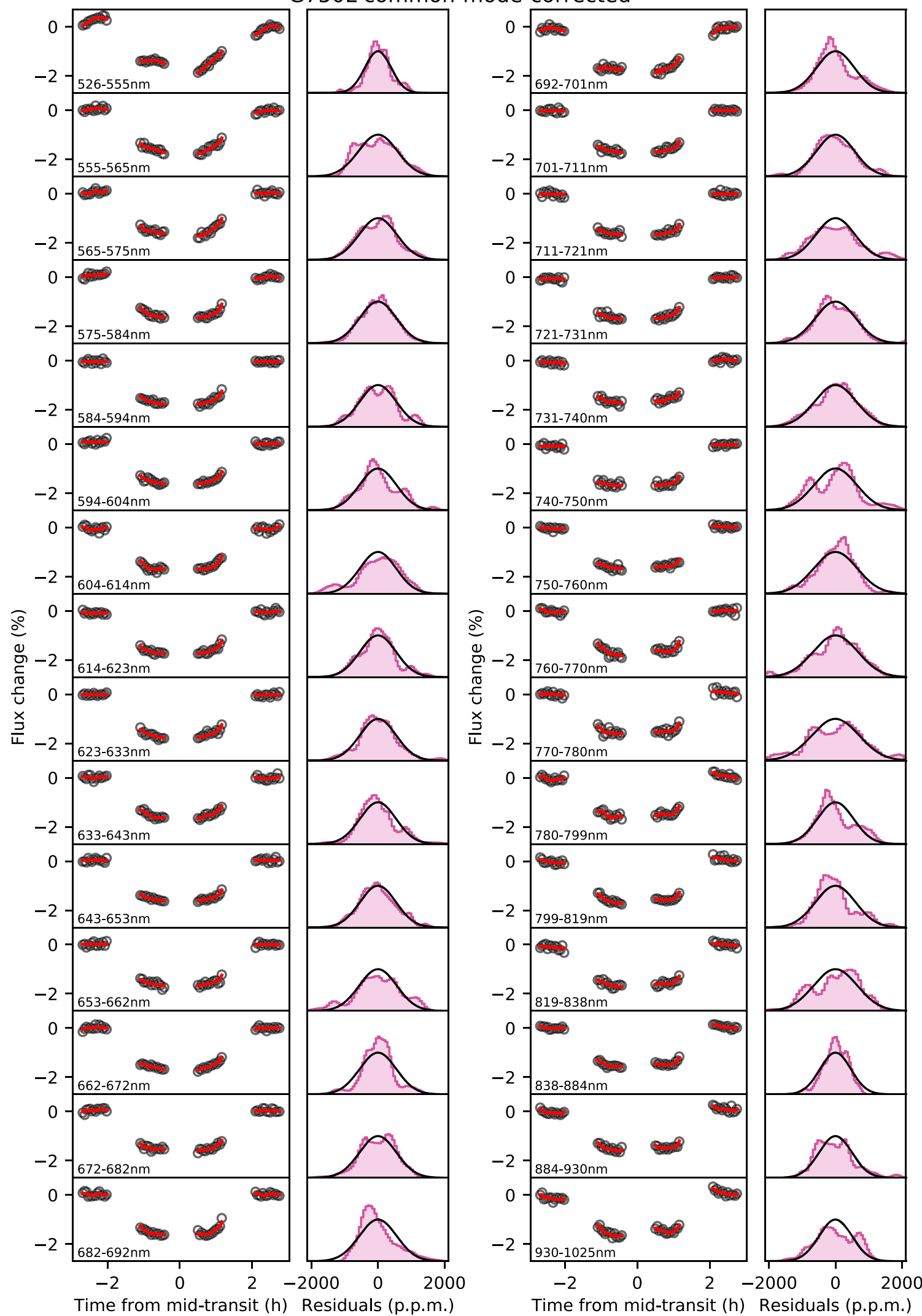

Figure 23. The same as Figure 21, but for the G750L data set. 


\section{Appendix B \\ Investigating the Robustness of the Measured Transmission Spectrum}

In this section, we consider a number of effects unrelated to the planet itself that could potentially introduce biases to the inferred transmission spectrum.

\section{B.1. Sensitivity to Limb-darkening Treatment}

As described in Sections 3 and 4, for our main light curve analyses, we adopted quadratic limb-darkening profiles and allowed both coefficients $\left(u_{1}, u_{2}\right)$ to vary as free parameters in the fitting. However, we also repeated the analyses using the four-parameter nonlinear law of Claret (2000), with coefficients fixed to values obtained by fitting to the limb-darkened profile of the STAGGER 3D stellar model described in Section 3. For the white light curve analyses, we found that the planet parameters inferred using the two limb-darkening treatments (i.e., "free quadratic" and "fixed nonlinear") were consistent to within $1 \sigma$, with only a single exception. Namely, for the fixed nonlinear analysis of the G750L light curve, we obtain $a / R_{\star}=3.59_{-0.13}^{+0.12}$ and $b=0.36_{-0.08}^{+0.07}$, which are both somewhat discrepant relative to the values inferred for the other light curves (Table 1).

For the spectroscopic light curve fits, the effect of the two limb-darkening treatments on the recovered transmission spectrum is illustrated in Figure 24. The differences are negligible for the G750L and G141 data sets. For the G430L data set, which at bluer wavelengths is more strongly affected by limb darkening, the transmission spectrum is systematically shifted to lower values for the fixed nonlinear analysis. Even so, the offset is less than $1 \sigma$ for almost all of the spectroscopic channels and does not affect the interpretation of the transmission spectrum. We therefore conclude that our results are insensitive to the choice of limb-darkening treatment.

\section{B.2. Including Time $t$ as a GP Input Variable}

We repeated the GP fits to the white light curves (as described in Section 3) and spectroscopic light curves (as described in Section 4) with time $t$ provided as a fourth input variable in addition to $\{\phi, x, y\}$. This was done to allow for possible departures from the linear function of $t$ that we assumed for the baseline trend. For instance, Demory et al. (2015) reported a ramp-like baseline trend for observations of Alpha Cen A spanning 16 and 9 consecutive HST orbits. We note, however, that Alpha Cen A has a brightness of $V=0 \mathrm{mag}$, compared to $V=10.5 \mathrm{mag}$ for WASP-121, which may result in especially pronounced systematics. We also note that analyses of STIS light curves often assume linear time baselines, and in a number of instances have been verified by independent observations using different instruments (e.g., Huitson et al. 2013; Fischer et al. 2016; Nikolov et al. 2016; Espinoza et al. 2018). Furthermore, in our experience of STIS light curves, baseline trend departures from a linear function of $t$ often correlate with $x$ and $y$, and would therefore be accounted for by the GP fits adopting only $\{\phi, x, y\}$ as input variables.

Given this, it is unsurprising that for the white light curve fits with $t$ included as an additional GP input, we obtain results consistent at the $1 \sigma$ level with those reported in Table 1 for all but one free parameter. The single exception is the $R_{\mathrm{p}} / R_{\star}$ value inferred from the joint analysis of the two G430L data sets, which was $0.1233_{-0.0006}^{+0.0006}$, compared with the value of

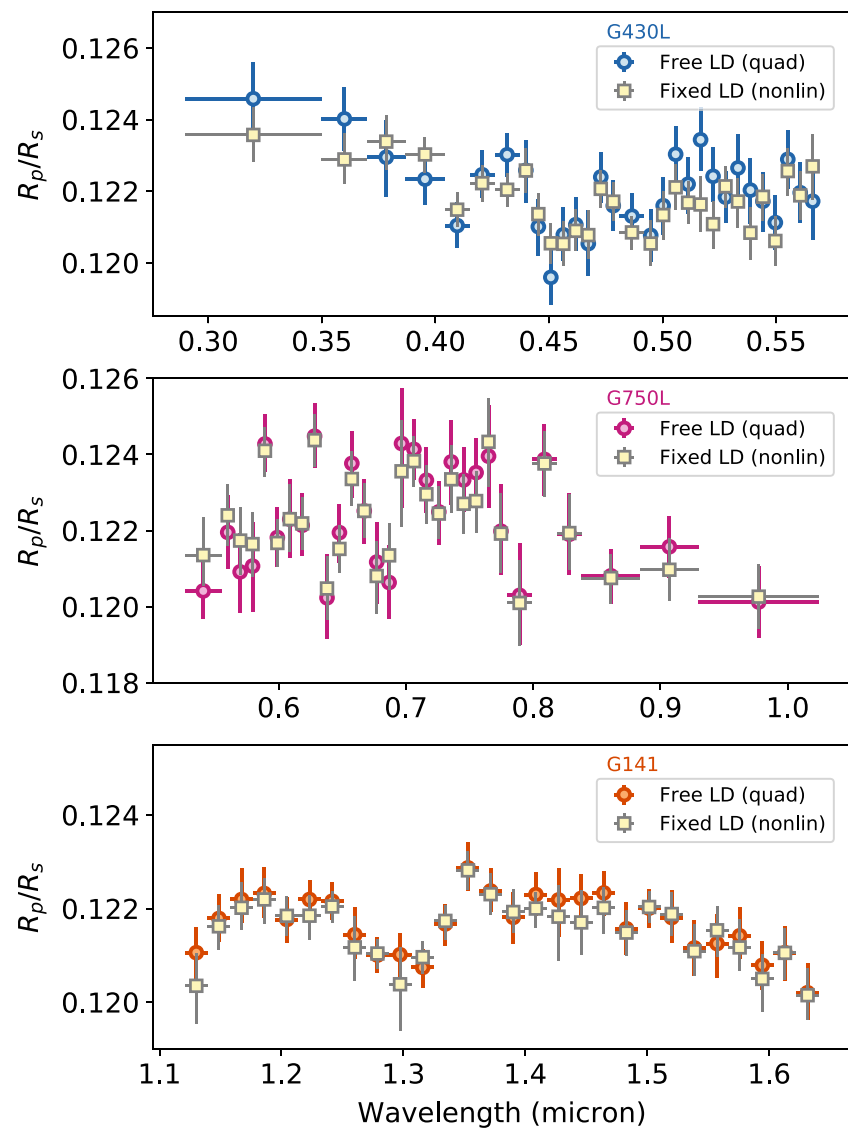

Figure 24. Sensitivity of inferred transmission spectrum to limb-darkening treatment for the G430L (top row), G750L (middle row), and G141 (bottom row) data sets. Colored circles show results obtained assuming a quadratic law with coefficients allowed to vary in the light curve fits and pale yellow squares show results obtained assuming a four-parameter nonlinear law with coefficients fixed to values estimated from the stellar model described in the text.

$0.1223_{-0.0006}^{+0.0006}$ obtained without $t$ as a GP input. However, this is not a statistically significant difference.

Similarly, for the spectroscopic light curve GP analyses including $t$ as an input, we obtain estimates for $R_{\mathrm{p}} / R_{\star}$ that are within $0.1 \sigma$ of those listed in Tables 2 and 3 for the majority of channels. Specifically, this was the case for 14 of the G430L channels and 26 of the G750L channels. For all remaining channels, the $R_{\mathrm{p}} / R_{\star}$ estimates were within $1 \sigma$ of those obtained without $t$ as a GP input. However, the uncertainties for $R_{\mathrm{p}} / R_{\star}$ increased by a median of $\sim 10 \%$ for both the G430L and G750L data sets when $t$ was included as a GP input. We plot the resulting transmission spectrum in Figure 25 and report the results in Table 5.

We suspect the inclusion of $t$ as an additional GP input results in overestimated uncertainties for $R_{\mathrm{p}} / R_{\star}$. For most channels, we found the inferred correlation length scale $L_{t}$ is large compared to $L_{\phi}, L_{x}$, and $L_{y}$, implying $t$ is a relatively unimportant input variable. In practice, $t$ likely plays a very similar role to $x$ and $y$ as a GP input (consider the second and third rows of Figure 2, after accounting for the repeatable orbitto-orbit variations in $x$ and $y$ ). Thus, by including $t$ as an input variable, we may be introducing an extra source of degeneracy to the systematics model, which is not justified by the data. This in turn could artificially broaden the posterior distribution for parameters such as $R_{\mathrm{p}} / R_{\star}$. For these reasons, we present the 
Table 5

Results of Spectroscopic Light Curve Fits with $t$ as an Additional GP Input for Selected Parameters

\begin{tabular}{|c|c|c|c|c|c|c|c|}
\hline \multicolumn{4}{|c|}{ G430L } & \multicolumn{4}{|c|}{ G750L } \\
\hline$\lambda(\AA)$ & $R_{\mathrm{p}} / R_{\star}$ & $u_{1}$ & $u_{2}$ & $\lambda(\AA)$ & $R_{\mathrm{p}} / R_{\star}$ & $u_{1}$ & $u_{2}$ \\
\hline 2898-3499 & $0.1246_{-0.0011}^{+0.0011}$ & $0.55_{-0.09}^{+0.10}$ & $0.21_{-0.15}^{+0.14}$ & $5263-5550$ & $0.1206_{-0.0009}^{+0.0011}$ & $0.37_{-0.08}^{+0.08}$ & $0.29_{-0.13}^{+0.13}$ \\
\hline 3700-3868 & $0.1242_{-0.0013}^{+0.0010}$ & $0.40_{-0.10}^{+0.10}$ & $0.39_{-0.16}^{+0.14}$ & $5648-5745$ & $0.1209_{-0.0012}^{+0.0011}$ & $0.36_{-0.09}^{+0.09}$ & $0.30_{-0.13}^{+0.14}$ \\
\hline $3868-4041$ & $0.1227_{-0.0009}^{+0.0009}$ & $0.56_{-0.09}^{+0.08}$ & $0.27_{-0.13}^{+0.14}$ & $5745-5843$ & $0.1212_{-0.0015}^{+0.0014}$ & $0.30_{-0.09}^{+0.09}$ & $0.32_{-0.14}^{+0.14}$ \\
\hline $4041-4151$ & $0.1215_{-0.0010}^{+0.0010}$ & $0.59_{-0.08}^{+0.09}$ & $0.16_{-0.14}^{+0.14}$ & 5843-5940 & $0.1243_{-0.0008}^{+0.0007}$ & $0.25_{-0.09}^{+0.08}$ & $0.28_{-0.13}^{+0.13}$ \\
\hline $4371-4426$ & $0.1224_{-0.0009}^{+0.00010}$ & $0.53_{-0.09}^{+0.09}$ & $0.23_{-0.13}^{+0.15}$ & $6135-6233$ & $0.1222_{-0.0009}^{+0.0009}$ & $0.20_{-0.08}^{+0.09}$ & $0.34_{-0.14}^{+0.13}$ \\
\hline $4426-4481$ & $0.1206_{-0.0007}^{+0.0008}$ & $0.64_{-0.09}^{+0.08}$ & $0.07_{-0.12}^{+0.13}$ & $6233-6330$ & $0.1245_{-0.0010}^{+0.0010}$ & $0.29_{-0.09}^{+0.08}$ & $0.20_{-0.13}^{+0.13}$ \\
\hline $4481-4536$ & $0.1196_{-0.0008}^{+0.0007}$ & $0.59_{-0.08}^{+0.07}$ & $0.18_{-0.12}^{+0.13}$ & $6330-6428$ & $0.1203_{-0.0012}^{+0.0012}$ & $0.31_{-0.09}^{+0.09}$ & $0.23_{-0.13}^{+0.13}$ \\
\hline $4536-4591$ & $0.1208_{-0.0008}^{+0.0008}$ & $0.49_{-0.08}^{+0.08}$ & $0.22_{-0.13}^{+0.13}$ & $6428-6526$ & $0.1220_{-0.0008}^{+0.0008}$ & $0.21_{-0.08}^{+0.09}$ & $0.22_{-0.13}^{+0.13}$ \\
\hline $4591-4646$ & $0.1211_{-0.0008}^{+0.0008}$ & $0.43_{-0.08}^{+0.08}$ & $0.30_{-0.13}^{+0.12}$ & $6526-6623$ & $0.1238_{-0.0009}^{+0.0009}$ & $0.21_{-0.09}^{+0.09}$ & $0.18_{-0.14}^{+0.13}$ \\
\hline $4921-4976$ & $0.1208_{-0.0009}^{+0.0009}$ & $0.43_{-0.09}^{+0.09}$ & $0.22_{-0.14}^{+0.13}$ & $7014-7111$ & $0.1241_{-0.0008}^{+0.0008}$ & $0.22_{-0.08}^{+0.08}$ & $0.20_{-0.13}^{+0.13}$ \\
\hline 4976-5030 & $0.1214_{-0.0010}^{+0.0009}$ & $0.45_{-0.09}^{+0.10}$ & $0.21_{-0.14}^{+0.15}$ & 7111-7209 & $0.1233_{-0.0009}^{+0.0009}$ & $0.15_{-0.09}^{+0.08}$ & $0.26_{-0.13}^{+0.13}$ \\
\hline $5030-5085$ & $0.1230_{-0.0008}^{+0.0008}$ & $0.40_{-0.08}^{+0.09}$ & $0.18_{-0.13}^{+0.14}$ & 7209-7307 & $0.1224_{-0.0009}^{+0.0008}$ & $0.22_{-0.08}^{+0.08}$ & $0.24_{-0.13}^{+0.13}$ \\
\hline $5085-5140$ & $0.1221_{-0.0008}^{+0.0009}$ & $0.49_{-0.09}^{+0.09}$ & $0.10_{-0.14}^{+0.14}$ & 7307-7404 & $0.1236_{-0.0011}^{+0.0012}$ & $0.19_{-0.10}^{+0.09}$ & $0.22_{-0.14}^{+0.14}$ \\
\hline $5140-5195$ & $0.1226_{-0.0013}^{+0.0012}$ & $0.38_{-0.09}^{+0.10}$ & $0.20_{-0.14}^{+0.14}$ & 7404-7502 & $0.1233_{-0.0009}^{+0.0009}$ & $0.07_{-0.09}^{+0.09}$ & $0.29_{-0.13}^{+0.13}$ \\
\hline $5195-5250$ & $0.1223_{-0.0007}^{+0.0008}$ & $0.35_{-0.08}^{+0.08}$ & $0.21_{-0.13}^{+0.13}$ & $7502-7600$ & $0.1236_{-0.0010}^{+0.0009}$ & $0.16_{-0.09}^{+0.09}$ & $0.16_{-0.13}^{+0.13}$ \\
\hline $5250-5305$ & $0.1217_{-0.0008}^{+0.0007}$ & $0.45_{-0.09}^{+0.09}$ & $0.21_{-0.14}^{+0.14}$ & $7600-7698$ & $0.1239_{-0.0020}^{+0.0019}$ & $0.21_{-0.10}^{+0.09}$ & $0.28_{-0.13}^{+0.14}$ \\
\hline $5305-5360$ & $0.1223_{-0.0010}^{+0.0010}$ & $0.39_{-0.10}^{+0.10}$ & $0.16_{-0.15}^{+0.14}$ & $7698-7795$ & $0.1220_{-0.0013}^{+0.0013}$ & $0.13_{-0.09}^{+0.09}$ & $0.31_{-0.13}^{+0.13}$ \\
\hline $5360-5415$ & $0.1221_{-0.0010}^{+0.0011}$ & $0.27_{-0.10}^{+0.10}$ & $0.27_{-0.15}^{+0.14}$ & 7795-7991 & $0.1204_{-0.0015}^{+0.0015}$ & $0.16_{-0.10}^{+0.10}$ & $0.26_{-0.15}^{+0.14}$ \\
\hline $5415-5469$ & $0.1214_{-0.0010}^{+0.0010}$ & $0.33_{-0.09}^{+0.09}$ & $0.32_{-0.14}^{+0.15}$ & $7991-8186$ & $0.1238_{-0.0012}^{+0.0010}$ & $0.16_{-0.09}^{+0.09}$ & $0.28_{-0.14}^{+0.13}$ \\
\hline $5469-5524$ & $0.1206_{-0.0009}^{+0.0009}$ & $0.36_{-0.09}^{+0.09}$ & $0.26_{-0.14}^{+0.14}$ & $8186-8381$ & $0.1220_{-0.0011}^{+0.0012}$ & $0.16_{-0.09}^{+0.09}$ & $0.24_{-0.13}^{+0.13}$ \\
\hline $5524-5579$ & $0.1228_{-0.0008}^{+0.0009}$ & $0.40_{-0.09}^{+0.09}$ & $0.16_{-0.13}^{+0.13}$ & $8381-8840$ & $0.1207_{-0.0009}^{+0.0008}$ & $0.09_{-0.09}^{+0.08}$ & $0.32_{-0.13}^{+0.13}$ \\
\hline
\end{tabular}

transmission spectrum reported in Section 5 as our nominal measurement and include this slightly more conservative analysis here for completeness. Under both analyses, our basic interpretation of the spectrum remains the same.

\section{B.3. Host Star Activity}

Host star activity in the form of dark and bright spots has the potential to introduce transit depth offsets between data sets, as well as chromatic biases within individual data sets. We have been monitoring WASP-121 with the Celestron 14 inch (C14) Automated Imaging Telescope (AIT) at Fairborn Observatory in southern Arizona (Henry 1999; Eaton et al. 2003). Observations were conducted over two campaigns using the Cousins $R$ photometric bandpass. The first campaign spanned 2017 January 27 to April 23, and the second campaign spanned 2018 February 22 to April 8. The CCD images were used to compute differential magnitudes with respect to the mean brightness of 10 of the most constant comparison stars in the same field. Further details of our data acquisition, reduction procedures, and analysis of the data can be found in Sing et al. (2015), which describes a similar monitoring program for the planetary host star WASP-31. Although our observations for WASP-121 were made after the $H S T$ transit observations, they allow us to constrain the photometric variability of the F6V

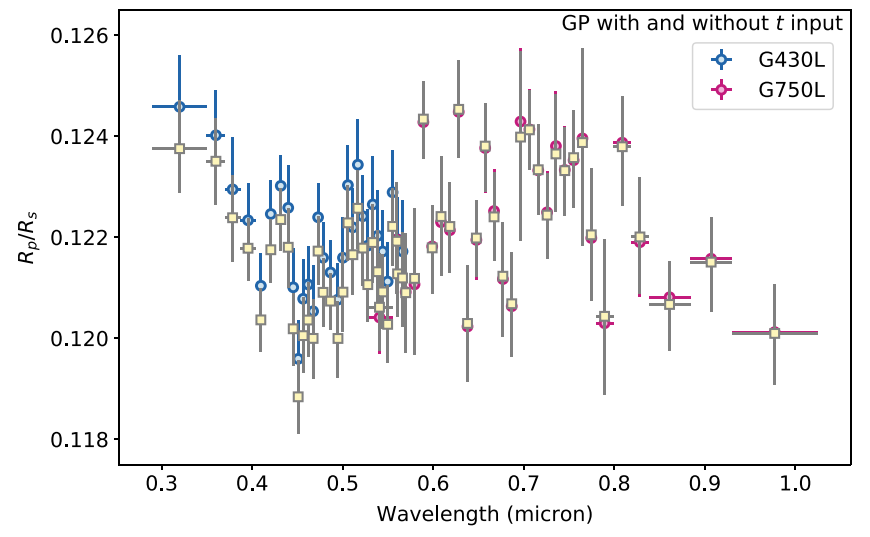

Figure 25. Comparison of the STIS transmission spectrum obtained with (pale yellow squares) and without (colored circles) time $t$ as a GP input variable.

host star over timescales spanning multiple stellar rotation periods.

Due to the southern declination of WASP-121 $\left(-39^{\circ} 05^{\prime} 51^{\prime \prime}\right)$ and the northern latitude of Fairborn Observatory $\left(+31^{\circ} 41^{\prime}\right.$ $18^{\prime \prime}$ ), the C14-AIT observations were made at large zenith angles between $70^{\circ}$ and $80^{\circ}$, corresponding to airmass values of 3-5. The panels in the first row of Figure 26 show the resulting photometry, after removing a small number of points 

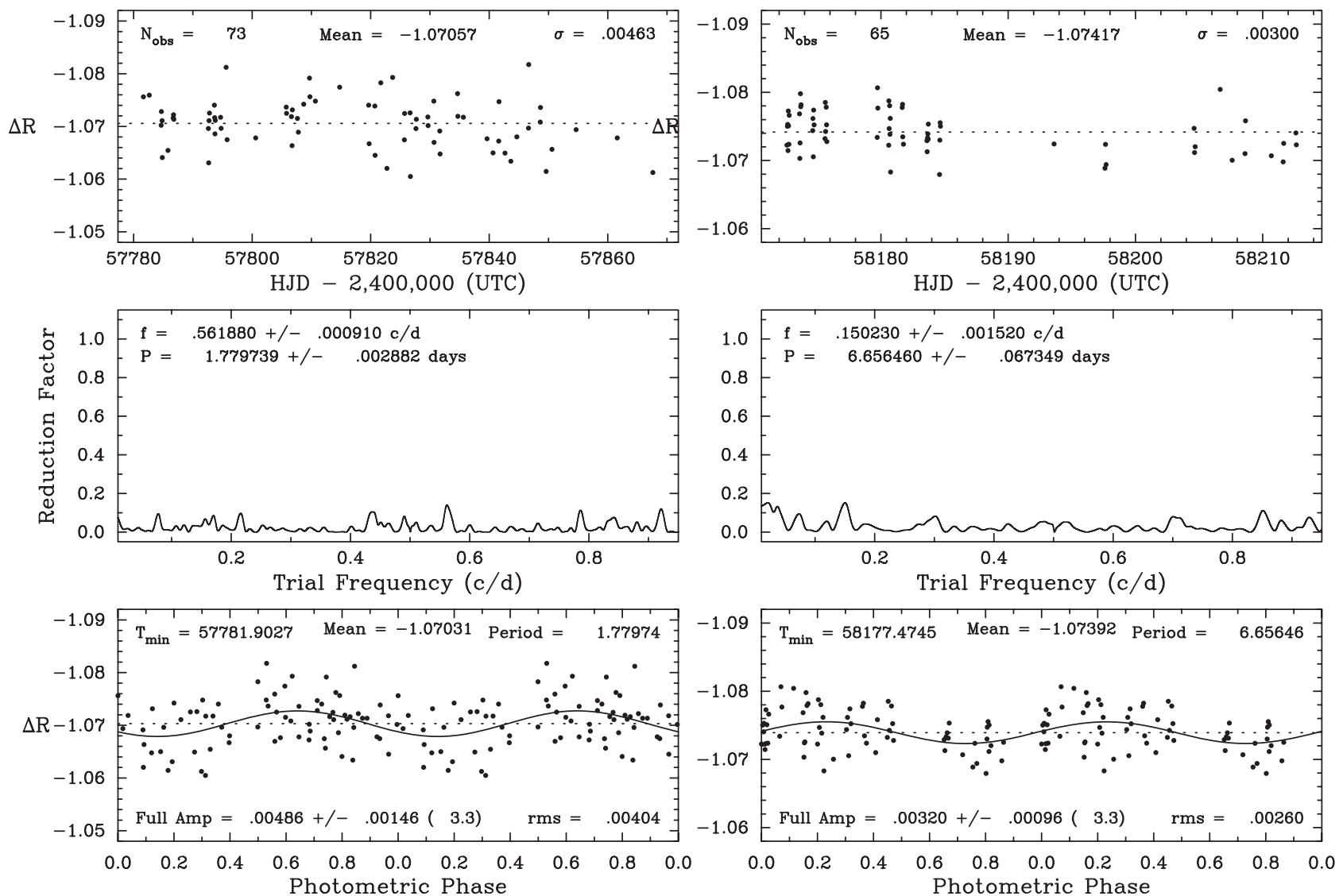

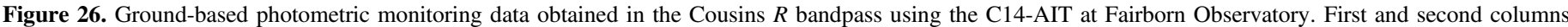

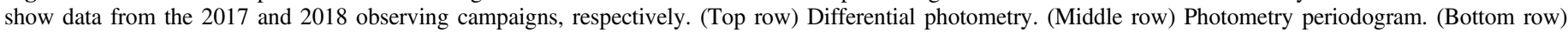

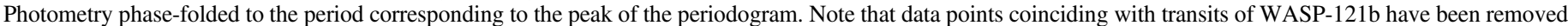
from these plots.

that coincided with transits of WASP-121b. We measure a mean differential brightness for WASP-121 relative to the comparison stars of -1.07057 mag for the 2017 campaign and $-1.07417 \mathrm{mag}$ for the 2018 campaign. The standard deviation about the yearly mean was found to be $4.6 \mathrm{mmag}$ for the 2017 campaign and $3.0 \mathrm{mmag}$ for the 2018 campaign. The telescope CCD was replaced between the 2017 and 2018 campaigns, which may explain the lower scatter in the 2018 campaign, as well as the $3.6 \mathrm{mmag}$ change in differential brightness. For comparison, Delrez et al. (2016) monitored WASP-121 over approximately six weeks using the TRAPPIST $60 \mathrm{~cm}$ telescope and reported standard deviations in the night-to-night photometry of $1.6 \mathrm{mmag}$ in the $B$ band, $1.3 \mathrm{mmag}$ in the $V$ band, and $1.1 \mathrm{mmag}$ in the $z^{\prime}$ band.

The second row of Figure 26 shows the frequency spectra for each C14-AIT campaign. The horizontal axis covers frequencies between 0.005 and $0.95 \mathrm{day}^{-1}$, corresponding to a period range of 1.05-200 days. No significant periodicity is detected for either campaign. In the third row of Figure 26, we plot the photometry phase-folded at a period of 1.78 days for the 2017 campaign and 6.66 days for the 2018 campaign, corresponding to the (insignificant) peaks of the respective periodograms. We obtain peak-to-peak amplitudes of $0.00486 \pm 0.00146$ mag for the 2017 campaign and $0.00320 \pm 0.00096 \mathrm{mag}$ for the 2018 campaign. In both cases, these amplitudes are comparable to the scatter in the residuals. A similar search for periodic signals in the WASP and TRAPPIST photometry performed by
Delrez et al. (2016) also failed to uncover any evidence for periodic signals above the $\sim 1 \mathrm{mmag}$ level. We therefore conclude that WASP-121 is photometrically stable over multiweek periods to at least the $5 \mathrm{mmag}$ level and likely to the $1 \mathrm{mmag}$ level or better. No significant periodicity has yet been detected and our ability to constrain the variability is currently limited by the available photometric precision.

The lack of detected photometric variability for WASP-121 implies transit depth measurements should not vary significantly from epoch-to-epoch due to intrinsic stellar activity. This is consistent with the good agreement we obtain for the two G430L visits (Appendix B.4) and also across the overlapping wavelength range covered by the G430L and G750L bandpasses (Appendix B.5). In addition, we observe no strong evidence for spot-crossing events in the transit light curves. However, a persistent, unocculted spot coverage could conceivably introduce chromatic biases to the measured transit depth while remaining undetected in the photometric monitoring data, due to the lack of time-varying signal. Given the apparently near-polar orientation of the planetary orbit (Delrez et al. 2016), the persistent spot coverage would not necessarily need to be uniform in longitude.

To quantify possible chromatic effects due to persistent unocculted spots, we follow a similar approach to that of Berta et al. (2011). First, for a star without spots, the out-of-transit flux $F_{\text {o.o.t. }}$ will be

$$
F_{\text {o.o.t. }}=A_{\star} f_{\star},
$$


where $A_{\star}$ is the area of the stellar disk and $f_{\star}$ is the stellar flux per unit area. Assuming a non-luminous nightside hemisphere for the planet and ignoring limb darkening, the measured intransit flux $F_{\text {i.t. }}$ will be

$$
F_{\text {i.t. }}=\left(A_{\star}-A_{\mathrm{p}}\right) f_{\star},
$$

where $A_{\mathrm{p}}$ is the area of the planet disk. This gives a relative transit depth $D=1-F_{\text {i.t. }} / F_{\text {o.o.t. }}$ of

$$
D=A_{\mathrm{p}} / A_{\star} \text {. }
$$

For a star with unocculted spots, the measured out-of-transit flux $\hat{F}_{\text {o.o.t. }}$ will be

$$
\hat{F}_{\text {o.o.t. }}=\left(A_{\star}-A_{\bullet}\right) f_{\star}+A \cdot f_{\bullet},
$$

where $A_{0}$ is the area covered by spots and $f_{\bullet}$ is the spot flux per unit area. The corresponding in-transit flux $\hat{F}_{\text {i.t. }}$ will be measured as

$$
\hat{F}_{\text {i.t. }}=\left(A_{\star}-A_{\bullet}-A_{\mathrm{p}}\right) f_{\star}+A_{\bullet} f_{\bullet} .
$$

It follows that the measured relative transit depth $\hat{D}=1-\hat{F}_{\text {i.t. }} / \hat{F}_{\text {o.o.t. }}$ will be

$$
\hat{D}=\frac{D}{1-\alpha[1-\beta(\lambda)]},
$$

where $\alpha \equiv A_{\bullet} / A_{\star}$ is the fractional area of the stellar disk covered by unocculted spots and $\beta(\lambda) \equiv f_{\bullet}(\lambda) / f_{\star}(\lambda)$ is the wavelength-dependent flux ratio of the spots and stellar photosphere. The chromatic bias $\kappa(\lambda)=\hat{D}-D$ due to unocculted spots will therefore be

$$
\kappa(\lambda)=D\left(\frac{\eta(\lambda)}{1-\eta(\lambda)}\right),
$$

where $\eta(\lambda) \equiv \alpha[1-\beta(\lambda)]$. This is equivalent to the spot corrections applied in previous studies such as Sing et al. (2011) and Huitson et al. (2013), and the transit light source effect described by Rackham et al. (2018).

Under the assumption that the true transit depth $D$ does not vary across the wavelength of interest, we fit the model given by Equation (6) to the transit depths derived from the measured $R_{\mathrm{p}} / R_{\star}$ values given in Table 2 . In these fits, we allowed $D$ and $\alpha$ to vary as free parameters, while for $f_{\star}(\lambda)$ we adopted a PHOENIX stellar model from the BT-Settl grid (Allard et al. $2012)$ with properties similar to WASP-121 $\left(T_{\star}=6500 \mathrm{~K}\right.$, $\log g=4.0 \mathrm{cgs},[\mathrm{Fe} / \mathrm{H}]=0 \mathrm{dex})$. For $f \cdot(\lambda)$, we used the same BT-Settl stellar model and repeated the fitting process for a range of assumed spot temperatures $T$. ranging from $6000 \mathrm{~K}$ down to $3500 \mathrm{~K}$ in increments of $500 \mathrm{~K}$. The results are shown in Figure 27.

If we restrict attention to the blue G430L data only, we find that unocculted spots can reproduce the shape of the measured spectrum. However, this requires invoking fractional coverages $\alpha$ ranging from $32 \%$ for $T_{\bullet}=5500 \mathrm{~K}$ to well over $50 \%$ for other $T$. values. Such large spot coverages would likely have a significant effect on the spectral typing of the star and be at odds with the modest X-ray flux we measure for WASP-121 (see below). Furthermore, the true transit depth $D$ would be no deeper than $1.2 \%$, which is significantly lower than the measured transit depths $\hat{D}$ of $>1.4 \%$ (Figure 27). We also find that the chromatic bias $\kappa(\lambda)$ given by Equation (7) would vary by $\Delta \kappa=2200 \mathrm{ppm}$ from the G430L to G141 bandpasses for $T_{.}=6000 \mathrm{~K}$, and by more for lower $T$. values. Instead, we measure consistent transit depths in the G430L and G141 bandpasses, to a precision of $\sim 200 \mathrm{ppm}$ (Table 1). Thus, under the unocculted spot scenario, the unbiased transit depth would be $>2200 \mathrm{ppm}$ deeper in the G141 bandpass relative to the G430L bandpass. This is equivalent to a change in the transmission spectrum of $>10 H$, where $H$ is a pressure scale height, since the change in measured transit depth due to $1 H$ is $\sim 150-200 \mathrm{ppm}$ for WASP-121b. Even larger differences would be expected for $T_{0}<6000 \mathrm{~K}$. Meanwhile, we are unable to reproduce the data if we attempt to fit the full STIS wavelength range using the same unocculted spot model (bottom panel of Figure 27). For these reasons, we consider it unlikely that unocculted spots can explain the measured spectrum.

Finally, we provide a brief report on measurements of WASP-121 made over approximately two hours on 2017 April 6 using the XMM-Newton space observatory (Obs ID 0804790601, P.I. Sanz-Forcada). Data were collected simultaneously at X-ray wavelengths $(0.12-2.48 \mathrm{keV}$; 5-100 А) with the XMM-EPIC instrument and UV wavelengths (1685-2480 ^) with the XMM-OM instrument. The X-ray fluxes, combined with the distance to the system (i.e., $272 \mathrm{pc}$; see Section 1), imply an X-ray luminosity $\log _{10} L_{X}=29.02$ (cgs). Using the multicolor brightness of WASP-121 ( $V=$ $10.51 \mathrm{mag}, B=11.0 \mathrm{mag}$ ) with the bolometric corrections of Flower (1996), we calculate a bolometric luminosity $\log _{10} L_{\mathrm{bol}}=$ 34.14 (cgs). This implies $\log _{10} L_{\mathrm{X}} / L_{\mathrm{bol}}=-5.12$, which is consistent with a low activity star. The XMM-OM UV time series does not show evidence for significant variability, while the XMM-EPIC X-ray time series may show some variability, although the statistics are poor and currently it is not possible to give a firm assessment. Further details will be provided in J. Sanz-Forcada et al. (2018, in preparation).

\section{B.4. Repeatability of the G430L Observations}

In Section 4, we presented the results of our primary G430L spectroscopic light curve analysis, for which $R_{\mathrm{p}} / R_{\star}$ was treated as a shared parameter fit jointly across both visits. However, we also analyzed each visit individually to check the measurement repeatability. The resulting transmission spectra are shown in the top panel of Figure 28 and exhibit good agreement. If we consider the median $R_{\mathrm{p}} / R_{\star}$ values inferred from the joint analysis to be the "ground truth," we can quantify the likelihood of measuring the transmission spectra for each individual visit using using $\chi_{\nu}^{2}=\left(\sum\left(\rho_{i}-\rho_{0, i}\right)^{2} / \sigma_{i}^{2}\right) / \nu$, where $\rho_{i}$ is the median $R_{\mathrm{p}} / R_{\star}$ value inferred for the $i$ th channel of the individual visit, with corresponding uncertainty $\sigma_{i} ; \rho_{0, i}$ is the corresponding $R_{\mathrm{p}} / R_{\star}$ value inferred from the joint analysis; and $\nu$ is the number of degrees of freedom, which in this case is equal to the number of spectroscopic channels. We obtain $\chi_{\nu}^{2}=1.0$ for G430Lv1 and $\chi_{\nu}^{2}=0.7$ for G430Lv2, implying that the transmission spectra inferred for each visit individually are consistent with being random draws of an underlying distribution centered at the $R_{\mathrm{p}} / R_{\star}$ values obtained from the joint analysis.

\section{B.5. Consistency of G430L-G750L Overlap}

There is some overlap between the G430L and G750L gratings, spanning approximately $0.55-0.57 \mu \mathrm{m}$ in wavelength (Figure 1). The transmission spectra recovered for both gratings 

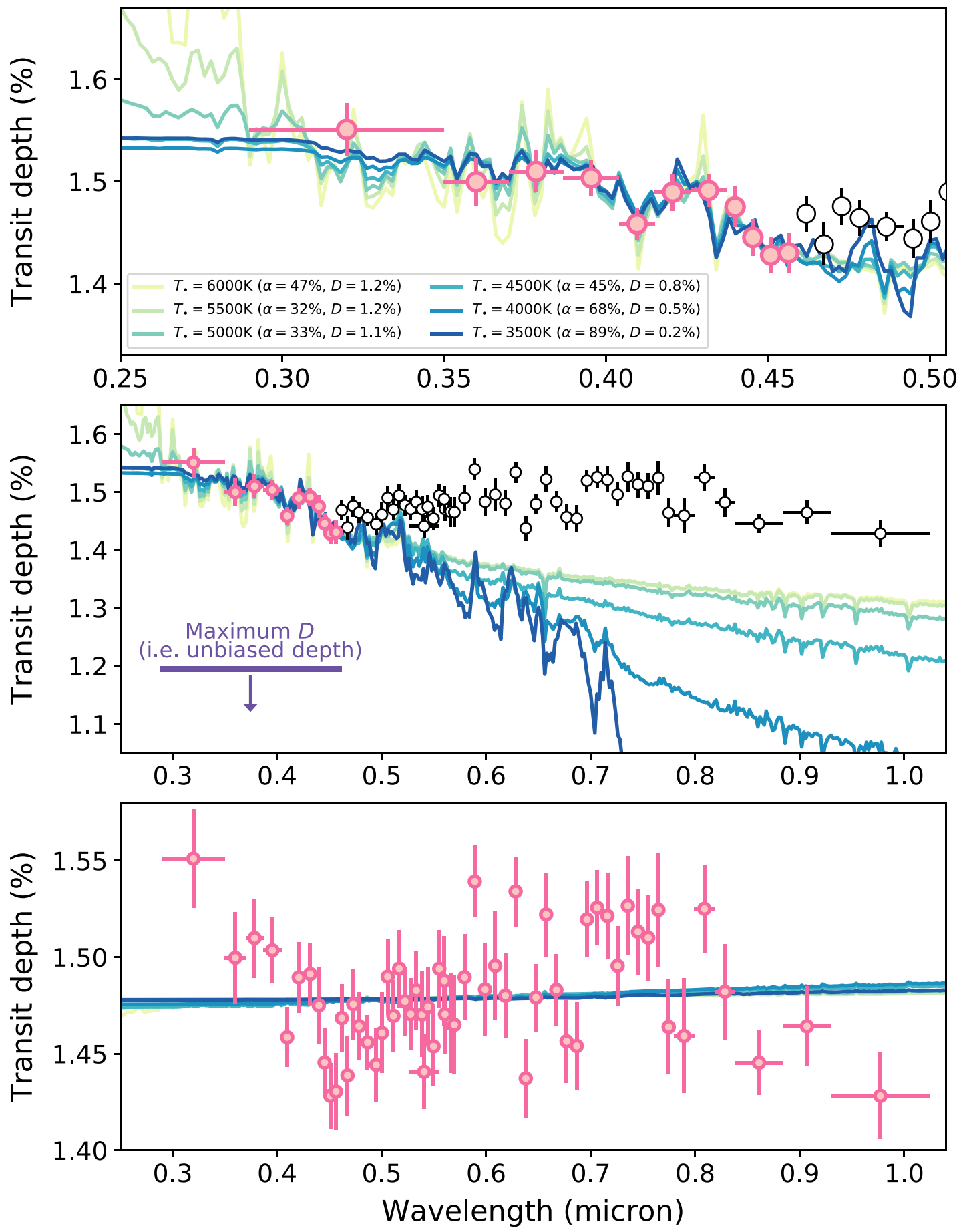

Figure 27. Fits to the data assuming transit depth variations are caused by chromatic biases due to unocculted star spots. In all panels, data used in the fit are indicated by pink circles and other data points are indicated by unfilled black circles. (Top panel) Fits to only the blue G430L data with inferred fractional spot coverages $\alpha$ and unbiased transit depths $D$ listed in the legend for different assumed spot temperatures. (Middle panel) The same, but over an extended wavelength range, to illustrate the predicted chromatic biases at longer wavelengths. Horizontal purple line indicates the implied range for the unbiased transit depths. (Bottom panel) Fits to the full STIS data set.

across this overlap region are consistent with each other, to within the measurement uncertainties (e.g., Figure 17). This gives some further reassurance that stellar variability or instrumental systematics have not introduced significant biases in the measured transit depth level from one observation to the next.

To test this more explicitly, we generated light curves spanning the full $0.55-0.57 \mu \mathrm{m}$ overlapping wavelength range for the G430Lv1, G430Lv2, and G750L data sets and fit them using the approach described in Section 4. A joint fit to the two G430L light curves gave $R_{\mathrm{p}} / R_{\star}=0.1225 \pm 0.0006$, while a fit to the G750L light curve gave $R_{\mathrm{p}} / R_{\star}=0.1216 \pm 0.0007$. Combining the uncertainties in quadrature, these results are consistent at the $1 \sigma$ level.

One possibility would be to apply a wavelength-uniform offset to either the G430L or G750L transmission spectrum, commensurate with the difference of $\sim 0.0009$ measured for $R_{\mathrm{p}} / R_{\star}$ across this overlapping wavelength range. The 


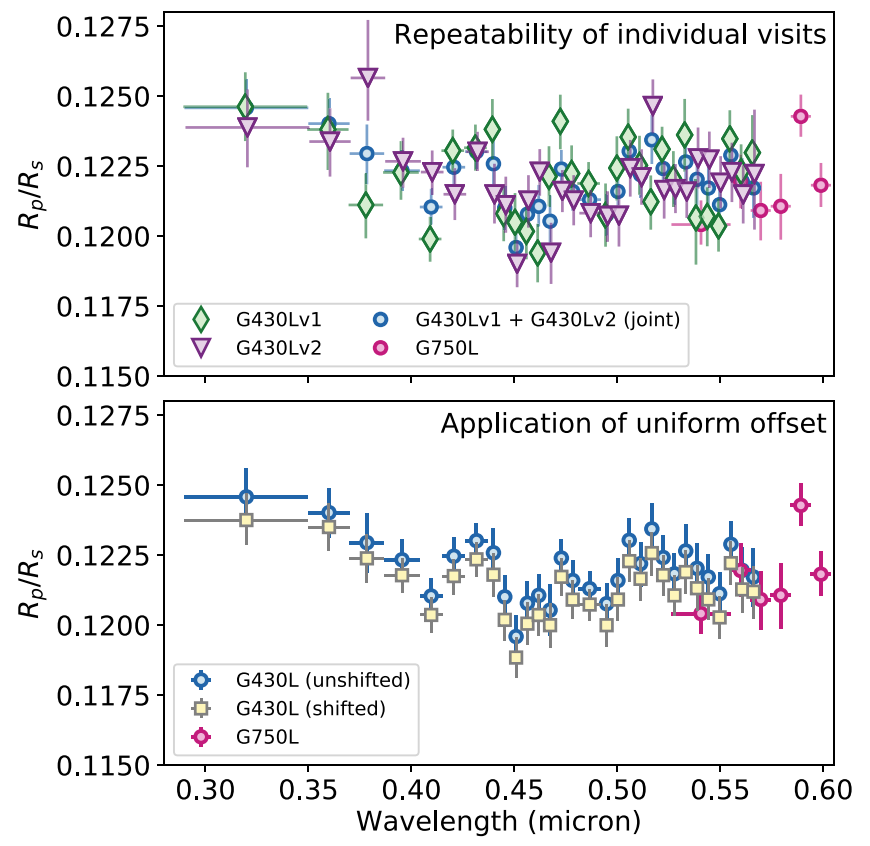

Figure 28. Measured transmission spectrum over the $0.3-0.6 \mu \mathrm{m}$ wavelength range, focusing on the G430L data set. (Top panel) Analyses of each visit individually (green triangles and purple diamonds) as well as the joint analysis of both visits simultaneously (filled blue circles). (Bottom panel) Application of a uniform vertical offset to align the overlapping range of the G430L and G750L gratings.

application of such an offset to the G430L spectrum is shown in the bottom panel of Figure 28. However, given the small amplitude of this offset relative to the measurement uncertainties, we found it did not affect our physical interpretation of the transmission spectrum. For example, the forward model described in Section 5.2, which assumes chemical equilibrium with $20 \times$ solar elemental abundances and a temperature of $T=1500 \mathrm{~K}$, is still compatible with the data (excluding the NUV rise and $1.15-1.3 \mu \mathrm{m}$ bump) with a reduced $\chi^{2}$ of 0.9 . Nonetheless, it is still worth emphasizing that the overall levels of the transmission spectrum subsets (i.e., G430L, G750L, G141) are each subject to some uncertainty, on the order of the corresponding white light curve $R_{\mathrm{p}} / R_{\star}$ uncertainty (Table 1 ).

\section{ORCID iDs}

Thomas M. Evans (iD https://orcid.org/0000-0001-5442-1300 David K. Sing (1) https://orcid.org/0000-0001-6050-7645 Nikolay Nikolov (D) https://orcid.org/0000-0002-6500-3574 Mark S. Marley (i) https://orcid.org/0000-0002-5251-2943 Kevin Zahnle (D) https://orcid.org/0000-0002-2462-4358 Gregory W. Henry (i) https://orcid.org/0000-0003-4155-8513 Joanna K. Barstow (D) https://orcid.org/0000-0003-3726-5419 Munazza K. Alam (iD https://orcid.org/0000-0003-4157-832X Jorge Sanz-Forcada (i) https://orcid.org/0000-0002-1600-7835 Tiffany Kataria (iD https://orcid.org/0000-0003-3759-9080 Nikole K. Lewis (i) https://orcid.org/0000-0002-8507-1304 Lotfi Ben-Jaffel (iD https://orcid.org/0000-0003-4047-2793 Sarah D. Blumenthal (iD https://orcid.org/0000-00023173-1637

Vincent Bourrier (iD https://orcid.org/0000-0002-9148-034X Benjamin Drummond (iD https://orcid.org/0000-00017589-5484

Antonio García Muñoz (iD https://orcid.org/0000-0003$1756-4825$
Mercedes López-Morales (ib https://orcid.org/0000-00033204-8183

Pascal Tremblin (iD https://orcid.org/0000-0001-6172-3403

David Ehrenreich (iD https://orcid.org/0000-0001-9704-5405

Hannah R. Wakeford (ib https://orcid.org/0000-00034328-3867

Lars A. Buchhave (i) https://orcid.org/0000-0003-1605-5666

Éric Hébrard (i) https://orcid.org/0000-0003-0770-7271

\section{References}

Allard, F., Homeier, D., \& Freytag, B. 2012, RSPTA, 370, 2765 Amundsen, D. S., Baraffe, I., Tremblin, P., et al. 2014, A\&A, 564, A59 Arcangeli, J., Désert, J.-M., Line, M. R., et al. 2018, ApJL, 855, L30 Asplund, M., Grevesse, N., Sauval, A. J., \& Scott, P. 2009, ARA\&A, 47, 481 Axel, L. 1972, ApJ, 173, 451

Beatty, T. G., Madhusudhan, N., Tsiaras, A., et al. 2017, AJ, 154, 158 Bell, T. J., Nikolov, N., Cowan, N. B., et al. 2017, ApJL, 847, L2 Ben-Jaffel, L., \& Ballester, G. E. 2013, A\&A, 553, A52

Berta, Z. K., Charbonneau, D., Bean, J., et al. 2011, ApJ, 736, 12 Burrows, A., \& Sharp, C. M. 1999, ApJ, 512, 843

Charbonneau, D., Brown, T. M., Noyes, R. W., \& Gilliland, R. L. 2002, ApJ, 568,377

Claret, A. 2000, A\&A, 363, 1081

Delrez, L., Santerne, A., Almenara, J.-M., et al. 2016, MNRAS, 458, 4025

Deming, D., Wilkins, A., McCullough, P., et al. 2013, ApJ, 774, 95

Demory, B.-O., Ehrenreich, D., Queloz, D., et al. 2015, MNRAS, 450, 2043

Drummond, B., Tremblin, P., Baraffe, I., et al. 2016, A\&A, 594, A69

Eastman, J., Gaudi, B. S., \& Agol, E. 2013, PASP, 125, 83

Eaton, J. A., Henry, G. W., \& Fekel, F. C. 2003, in The Future of Small Telescopes In The New Millennium. Volume II - The Telescopes We Use, ed. T. D. Oswalt (Dordrecht: Kluwer), 189

Ehrenreich, D., Bourrier, V., Wheatley, P. J., et al. 2015, Natur, 522, 459 Espinoza, N., Rackham, B. V., Jordán, A., et al. 2018, MNRAS, 482, 2065 Esposito, L. W., Bertaux, J.-L., Krasnopolsky, V., Moroz, V. I., \& Zasova, L. V. 1997, in Venus II: Geology, Geophysics, Atmosphere, and Solar Wind Environment, ed. S. W. Bougher, D. M. Hunten, \& R. J. Phillips (Tucson, AZ: Univ. Arizona Press), 415

Evans, T. M., Pont, F., Sing, D. K., et al. 2013, ApJL, 772, L16

Evans, T. M., Sing, D. K., Kataria, T., et al. 2017, Natur, 548, 58

Evans, T. M., Sing, D. K., Wakeford, H. R., et al. 2016, ApJL, 822, L4

Fischer, P. D., Knutson, H. A., Sing, D. K., et al. 2016, ApJ, 827, 19

Flower, P. J. 1996, ApJ, 469, 355

Foreman-Mackey, D., Hogg, D. W., Lang, D., \& Goodman, J. 2013, PASP, 125,306

Fortney, J. J. 2005, MNRAS, 364, 649

Fortney, J. J., Lodders, K., Marley, M. S., \& Freedman, R. S. 2008, ApJ, 678,1419

Fossati, L., Haswell, C. A., Froning, C. S., et al. 2010, ApJL, 714, L222

Fraine, J., Deming, D., Benneke, B., et al. 2014, Natur, 513, 526

Gaia Collaboration, Brown, A. G. A., Vallenari, A., et al. 2018, A\&A, 616, A1 Gelman, A., \& Rubin, D. B. 1992, StaSc, 7, 457

Gibson, N. P. 2014, MNRAS, 445, 3401

Gibson, N. P., Aigrain, S., Roberts, S., et al. 2012, MNRAS, 419, 2683

Gibson, N. P., Nikolov, N., Sing, D. K., et al. 2017, MNRAS, 467, 4591

Goyal, J. M., Mayne, N., Sing, D. K., et al. 2018, MNRAS, 474, 5158

Haynes, K., Mandell, A. M., Madhusudhan, N., Deming, D., \& Knutson, H. 2015, ApJ, 806, 146

Henry, G. W. 1999, PASP, 111, 845

Hubeny, I., Burrows, A., \& Sudarsky, D. 2003, ApJ, 594, 1011

Huitson, C. M., Sing, D. K., Pont, F., et al. 2013, MNRAS, 434, 3252

Huitson, C. M., Sing, D. K., Vidal-Madjar, A., et al. 2012, MNRAS, 422, 2477

Kataria, T., Sing, D. K., Lewis, N. K., et al. 2016, ApJ, 821, 9

Kempton, E. M.-R., Bean, J. L., \& Parmentier, V. 2017, ApJL, 845, L20

Kreidberg, L., Bean, J. L., Désert, J.-M., et al. 2014, Natur, 505, 69

Kreidberg, L., Line, M. R., Bean, J. L., et al. 2015, ApJ, 814, 66

Kreidberg, L., Line, M. R., Parmentier, V., et al. 2018, AJ, 156, 17

Lecavelier Des Etangs, A., Pont, F., Vidal-Madjar, A., \& Sing, D. 2008, A\&A, 481, L83

Lodders, K. 2002, ApJ, 577, 974

Lothringer, J. D., Barman, T., \& Koskinen, T. 2018, ApJ, 866, 27

Madhusudhan, N., Burrows, A., \& Currie, T. 2011, ApJ, 737, 34

Magic, Z., Collet, R., Asplund, M., et al. 2013, A\&A, 557, A26

Mandel, K., \& Agol, E. 2002, ApJL, 580, L171 
Mansfield, M., Bean, J. L., Line, M. R., et al. 2018, AJ, 156, 10

Marley, M. S., \& McKay, C. P. 1999, Icar, 138, 268

Marley, M. S., Saumon, D., Cushing, M., et al. 2012, ApJ, 754, 135

Marley, M. S., Seager, S., Saumon, D., et al. 2002, ApJ, 568, 335

Mbarek, R., \& Kempton, E. M.-R. 2016, ApJ, 827, 121

Nikolov, N., Sing, D. K., Burrows, A. S., et al. 2015, MNRAS, 447, 463

Nikolov, N., Sing, D. K., Gibson, N. P., et al. 2016, ApJ, 832, 191

Nikolov, N., Sing, D. K., Goyal, J., et al. 2018, MNRAS, 474, 1705

Nikolov, N., Sing, D. K., Pont, F., et al. 2014, MNRAS, 437, 46

Nugroho, S. K., Kawahara, H., Masuda, K., et al. 2017, AJ, 154, 221

Owen, T., \& Sagan, C. 1972, Icar, 16, 557

Parmentier, V., Line, M. R., Bean, J. L., et al. 2018, A\&A, 617, A110

Parmentier, V., Showman, A. P., \& Lian, Y. 2013, A\&A, 558, A91

Pollack, J. B., Toon, O. B., Whitten, R. C., et al. 1980, JGR, 85, 8141

Pont, F., Knutson, H., Gilliland, R. L., Moutou, C., \& Charbonneau, D. 2008 , MNRAS, 385, 109

Rackham, B. V., Apai, D., \& Giampapa, M. S. 2018, ApJ, 853, 122

Saumon, D., \& Marley, M. S. 2008, ApJ, 689, 1327

Seager, S., \& Sasselov, D. D. 1998, ApJL, 502, L157

Seager, S., \& Sasselov, D. D. 2000, ApJ, 537, 916

Sedaghati, E., Boffin, H. M. J., MacDonald, R. J., et al. 2017, Natur, 549, 238

Sharp, C. M., \& Burrows, A. 2007, ApJS, 168, 140

Showman, A. P., Fortney, J. J., Lian, Y., et al. 2009, ApJ, 699, 564

Sing, D. K., Fortney, J. J., Nikolov, N., et al. 2016, Natur, 529, 59

Sing, D. K., Pont, F., Aigrain, S., et al. 2011, MNRAS, 416, 1443
Sing, D. K., Wakeford, H. R., Showman, A. P., et al. 2015, MNRAS, 446, 2428

Spake, J. J., Sing, D. K., Evans, T. M., et al. 2018, Natur, 557, 68

Spiegel, D. S., Silverio, K., \& Burrows, A. 2009, ApJ, 699, 1487

Stevenson, K. B., Désert, J.-M., Line, M. R., et al. 2014, Sci, 346, 838

Thorngren, D. P., Fortney, J. J., Murray-Clay, R. A., \& Lopez, E. D. 2016, ApJ, 831, 64

Tremblin, P., Amundsen, D. S., Chabrier, G., et al. 2016, ApJL, 817, L19

Tremblin, P., Amundsen, D. S., Mourier, P., et al. 2015, ApJL, 804, L17

Tsiaras, A., Waldmann, I. P., Zingales, T., et al. 2018, AJ, 155, 156

Vidal-Madjar, A., Lecavelier des Etangs, A., Désert, J.-M., et al. 2003, Natur, 422,143

Visscher, C., Lodders, K., \& Fegley, B., Jr. 2006, ApJ, 648, 1181

Wakeford, H. R., Sing, D. K., Deming, D., et al. 2018, AJ, 155, 29

Wakeford, H. R., Sing, D. K., Kataria, T., et al. 2017, Sci, 356, 628

West, R. A., Baines, K. H., Friedson, A. J., et al. 2004, in Jupiter. The planet, satellites and magnetosphere., ed. F. Bagenal, T. E. Dowling, \& W. B. McKinnon (Cambridge: Cambridge Univ. Press), 79

Woitke, P., Helling, C., Hunter, G. H., et al. 2018, A\&A, 614, A1

Wyttenbach, A., Ehrenreich, D., Lovis, C., Udry, S., \& Pepe, F. 2015, A\&A, 577, A62

Yurchenko, S. N., Bond, W., Gorman, M. N., et al. 2018, MNRAS, 478, 270

Zahnle, K., Marley, M. S., Freedman, R. S., Lodders, K., \& Fortney, J. J. 2009, ApJL, 701, L20

Zahnle, K., Marley, M. S., Morley, C. V., \& Moses, J. I. 2016, ApJ, 824, 137 